\title{
Compound conditionals, Fréchet-Hoeffding bounds, and Frank t-norms
}

\author{
Angelo Gilio ${ }^{\mathrm{a}, 1,2}$, Giuseppe Sanfilippo ${ }^{\mathrm{b}, 3, *}$ \\ ${ }^{a}$ Department of Basic and Applied Sciences for Engineering, University of Rome "La Sapienza", Via A. Scarpa 14, 00161 Roma, \\ Italy \\ ${ }^{b}$ Department of Mathematics and Computer Science, Via Archirafi 34, 90123 Palermo, Italy
}

\begin{abstract}
In this paper we consider compound conditionals, Fréchet-Hoeffding bounds and the probabilistic interpretation of Frank t-norms. By studying the solvability of suitable linear systems, we show under logical independence the sharpness of the Fréchet-Hoeffding bounds for the prevision of conjunctions and disjunctions of $n$ conditional events. In addition, we illustrate some details in the case of three conditional events. We study the set of all coherent prevision assessments on a family containing $n$ conditional events and their conjunction, by verifying that it is convex. We discuss the case where the prevision of conjunctions is assessed by Lukasiewicz t-norms and we give explicit solutions for the linear systems; then, we analyze a selected example. We obtain a probabilistic interpretation of Frank t-norms and t-conorms as prevision of conjunctions and disjunctions of conditional events, respectively. Then, we characterize the sets of coherent prevision assessments on a family containing $n$ conditional events and their conjunction, or their disjunction, by using Frank t-norms, or Frank t-conorms. By assuming logical independence, we show that any Frank t-norm (resp., t-conorm) of two conditional events $A \mid H$ and $B \mid K, T_{\lambda}(A|H, B| K)\left(\operatorname{resp} ., S_{\lambda}(A|H, B| K)\right)$, is a conjunction $(A \mid H) \wedge(B \mid K)$ (resp., a disjunction $(A \mid H) \vee(B \mid K)$ ). Then, we analyze the case of logical dependence where $A=B$ and we obtain the set of coherent assessments on $A|H, A| K,(A \mid H) \wedge(A \mid K)$; moreover we represent it in terms of the class of Frank t-norms $T_{\lambda}$, with $\lambda \in[0,1]$. By considering a family $\mathcal{F}$ containing three conditional events, their conjunction, and all pairwise conjunctions, we give some results on Frank t-norms and coherence of the prevision assessments on $\mathcal{F}$. By assuming logical independence, we show that it is coherent to assess the previsions of all the conjunctions by means of Minimum and Product t-norms. In this case all the conjunctions coincide with the t-norms of the corresponding conditional events. We verify by a counterexample that, when the previsions of conjunctions are assessed by the Lukasiewicz tnorm, coherence is not assured. Then, the Lukasiewicz t-norm of conditional events may not be interpreted as their conjunction. Finally, we give two sufficient conditions for coherence and incoherence when using the Lukasiewicz t-norm.
\end{abstract}

Keywords: Coherence, Conditional previsions, Convexity, Conjunction and disjunction, Fréchet-Hoeffding bounds, Frank t-norms

\footnotetext{
* Corresponding author

Email addresses: angelo.gilio@sbai .uniroma1.it (Angelo Gilio), giuseppe.sanfilippo@unipa.it (Giuseppe Sanfilippo)

${ }^{1}$ Both authors equally contributed to this work

${ }^{2}$ Retired

${ }^{3}$ Also affiliated with INdAM-GNAMPA, Italy

International Journal of Approximate Reasoning
} 


\section{Introduction}

In this paper we consider conjunctions and disjunctions of conditional events. These compound conditionals are defined in the setting of coherence as suitable conditional random quantities, with values in the unit interval (see, e.g. [27, 28, 29, 30, 33, 34, 52, 53]). In [30] we proved the sharpness of the FréchetHoeffding bounds for the prevision of the conjunction and disjunction of two conditional events. We recall that such lower and upper bounds are particular Frank t-norms and t-conorms: for the conjunction they are the Lukasiewicz and Minimum t-norms, respectively; for the disjunction they are the dual t-conorms. In this paper we generalize this result to the conjunction $\mathcal{C}_{1 \ldots n}$ and the disjunction $\mathcal{D}_{1 \ldots n}$ of $n$ conditional events $E_{1}\left|H_{1}, \ldots, E_{n}\right| H_{n}$. To obtain this result we study the solvability of suitable linear systems associated with prevision assessments $\mathcal{M}$ on the family $\left\{E_{1}\left|H_{1}, \ldots, E_{n}\right| H_{n}, \mathcal{C}_{1 \ldots n}\right\}$. We provide some explicit solutions for the linear systems and we show that the set of coherent assessments $\mathcal{M}$ is convex. To better illustrate our results, we examine more details in the case of three conditional events.

We discuss the case where the prevision of conjunctions is assessed by Lukasiewicz t-norms and we give explicit solutions for the linear systems; then, we analyze a selected example. We give a probabilistic interpretation of Frank t-norms and t-conorms as prevision of conjunction and disjunction of conditional events, respectively. Then, we characterize the sets of coherent prevision assessments on the families $\left\{E_{1}\left|H_{1}, \ldots, E_{n}\right| H_{n}, \mathcal{C}_{1 \cdots n}\right\}$ and $\left\{E_{1}\left|H_{1}, \ldots, E_{n}\right| H_{n}, \mathcal{D}_{1 \cdots n}\right\}$ in terms of Frank t-norms and Frank t-conorms, respectively. In addition, by assuming logical independence, we show that any Frank t-norm of two conditional events $A \mid H$ and $B \mid K, T_{\lambda}(A|H, B| K)$, is the conjunction $(A \mid H) \wedge(B \mid K)$ associated with the assessment $\mathbb{P}[(A \mid H) \wedge(B \mid K)]=T_{\lambda}(P(A \mid H), P(B \mid K))$. A dual result is given for the disjunction in terms of dual t-conorm.

We analyze the case of logical dependence where $A=B$ and we determine the set of all coherent assessments $(x, y, z)$ on $\{A|H, A| K,(A \mid H) \wedge(A \mid K)\}$, by also showing that $T_{\lambda}(A|H, A| K)$ represents a conjunction $(A \mid H) \wedge(A \mid K)$, only for $\lambda \in[0,1]$. In particular, when $H K=\varnothing$, we obtain that $(A \mid H) \wedge(A \mid K)$ coincides with the Product t-norm $T_{1}(A|H, A| K)=(A \mid H) \cdot(A \mid K)$.

Given three conditional events, we consider all possible conjunctions among them and we show that to make prevision assignments on conjunctions by means of the Product t-norm, or the Minimum t-norm, is coherent. Moreover, the conjoined conditionals can be represented as Product t-norms, or Minimum t-norms, of the involved conditional events. This representation may not hold for the Lukasiewicz t-norm. Indeed, we show by a counterexample that prevision assignments on conjunctions by means of the Lukasiewicz t-norm may be not coherent and we examine some sufficient conditions for coherence and incoherence. Finally, we give two sufficient conditions for coherence and incoherence when using the Lukasiewicz t-norm.

A relevant aspect which would deserve investigation is the application of our results on compound conditionals and t-norms in statistical matching, misclassified data, data fusion, aggregation operators, fuzzy logic, belief and plausibility functions, and description logic ([1, 5, 9, 10, 15, 16, 17, 18, 19, 20, 36, 47, 49]). This paper originated from [32] and the large part of the material is new. In particular all the results given in Section 3, Section 4, and Subsection 5.1 are new. Revised and extended material from [32] is given in Subsections 5.2 and 5.3 , and Section 6 .

The paper is organized as follows: In Section 2 we recall some preliminary notions and theoretical results on conditional random quantities and coherence. We give some examples and we examine an extended notion of conditional random quantity $X \mid H$. We recall compound conditionals and Frank t-norms. In Section 3 , by studying the solvability of suitable linear systems, we show under logical independence the sharpness of Fréchet-Hoeffding bounds for the prevision of the conjunction $\mathcal{C}_{1 \cdots n}$ of $n$ conditional events; we illustrate more details in the case $n=3$. We also give a geometrical characterization of the set $\Pi$ of all coherent prevision assessments on $\mathcal{F}=\left\{E_{1}\left|H_{1}, \ldots, E_{n}\right| H_{n}, \mathcal{C}_{1 \ldots n}\right\}$, by showing that $\Pi$ is convex. In Section 
4 we examine in detail the case where the prevision of the conjunction is assessed by means of Lukasiewicz t-norm and we analyze a selected example. In Section 5 we study the representation of the prevision, for the conjunction $\mathcal{C}_{1 \cdots n}$ and the disjunction $\mathcal{D}_{1 \ldots n}$ of $n$ conditional events, as a Frank t-norm $T_{\lambda}$ and a Frank t-conorm $S_{\lambda}$, respectively. Then, by exploiting Frank t-norms and t-conorms, we characterize the sets of coherent prevision assessments on $\left\{E_{1}\left|H_{1}, \ldots, E_{n}\right| H_{n}, \mathcal{C}_{1 \ldots n}\right\}$ and on $\left\{E_{1}\left|H_{1}, \ldots, E_{n}\right| H_{n}, \mathcal{D}_{1 \ldots n}\right\}$. We show that, under logical independence, $T_{\lambda}(A|H, B| K)=(A \mid H) \wedge(B \mid K)$ and $S_{\lambda}(A|H, B| K)=(A \mid H) \vee(B \mid K)$ for every $\lambda \in[0,+\infty]$. We also examine the case of logical dependence where $A=B$ and the particular case where $H K=\varnothing$. In Section 6 we give some particular results on Frank t-norms and coherence of prevision assessments on the family $\mathcal{F}=\left\{E_{1}\left|H_{1}, E_{2}\right| H_{2}, E_{3} \mid H_{3},\left(E_{1} \mid H_{1}\right) \wedge\left(E_{2} \mid H_{2}\right),\left(E_{1} \mid H_{1}\right) \wedge\left(E_{3} \mid H_{3}\right),\left(E_{2} \mid H_{2}\right) \wedge\right.$ $\left.\left(E_{3} \mid H_{3}\right),\left(E_{1} \mid H_{1}\right) \wedge\left(E_{2} \mid H_{2}\right) \wedge\left(E_{3} \mid H_{3}\right)\right\}$. In particular, we show that, under logical independence, the assessment $\mathcal{M}=\left(x_{1}, x_{2}, x_{3}, T_{\lambda}\left(x_{1}, x_{2}\right), T_{\lambda}\left(x_{1}, x_{3}\right), T_{\lambda}\left(x_{2}, x_{3}\right), T_{\lambda}\left(x_{1}, x_{2}, x_{3}\right)\right)$ on $\mathcal{F}$ is coherent for every $\left(x_{1}, x_{2}, x_{3}\right) \in[0,1]^{3}$ when $T_{\lambda}$ is the minimum t-norm, or the product t-norm. Moreover, when $T_{\lambda}$ is the Lukasiewicz t-norm, the coherence of $\mathcal{M}$ is not assured and hence it may happen that the Frank t-norm of three conditional events is not a conjunction. Finally, we give some sufficient conditions for coherence/incoherence of $\mathcal{M}$ when using the Lukasiewicz t-norm. In Section 7 we give some conclusions.

\section{Preliminary notions and results}

In this section we recall some basic notions and results which concern conditional events, conditional random quantities, coherence (see, e.g., [2, 3, 4, 7, 12, 26, 43, 46, 48]), and logical operations among conditional events (see [28, 29, 30, 31, 33, 35]).

\subsection{Conditional events, conditional random quantities, and coherent prevision assessments}

Uncertainty about unknown facts is formalized by events. In formal terms, an event $E$ is a two-valued logical entity which can be true, or false. The indicator of $E$, denoted by the same symbol, is 1 , or 0 , according to whether $E$ is true, or false. Thus, a symbol like $x E$ represents the product of the quantity $x$ and the indicator of the event $E$. The sure event and impossible event are denoted by $\Omega$ and $\varnothing$, respectively. Given two events $E_{1}$ and $E_{2}$, we denote by $E_{1} \wedge E_{2}$, or simply by $E_{1} E_{2}$, (resp., $E_{1} \vee E_{2}$ ) the logical conjunction (resp., the logical disjunction). The negation of $E$ is denoted $\bar{E}$. We simply write $E_{1} \subseteq E_{2}$ to denote that $E_{1}$ logically implies $E_{2}$, that is $E_{1} \bar{E}_{2}=\varnothing$. We recall that $n$ events $E_{1}, \ldots, E_{n}$ are logically independent when the number $m$ of constituents, or possible worlds, generated by them is $2^{n}$.

Given two events $E, H$, with $H \neq \varnothing$, the conditional event $E \mid H$ is defined as a three-valued logical entity which is true, or false, or void, according to whether $E H$ is true, or $\bar{E} H$ is true, or $\bar{H}$ is true, respectively.

Given a (real) random quantity $X$ and an event $H \neq \varnothing$, we denote by $\mathbb{P}(X \mid H)$ the prevision of $X$ conditional on $H$, with $\mathbb{P}(X \mid H)=P(E \mid H)$ when $X$ is (the indicator of) an event $E$. In what follows, for any given conditional random quantity $X \mid H$, we assume that, when $H$ is true, the set of possible values of $X$ is a finite subset of the set of real numbers $\mathbb{R}$. In this case we say that $X \mid H$ is a finite conditional random quantity. In the framework of coherence, to assess $\mathbb{P}(X \mid H)=\mu$ means that, for every real number $s$, you are willing to pay an amount $s \mu$ and to receive $s(X H+\mu \bar{H})$, that is to receive $s X$, or $s \mu$, according to whether $H$ is true, or $\bar{H}$ is true (bet called off), respectively. The random gain is $G=s(X H+\mu \bar{H})-s \mu=s H(X-\mu)$. In particular, given any conditional event $E \mid H$, if we assess $P(E \mid H)=x$, then the random gain is $G=s H(E-x)$.

Given a prevision function $\mathbb{P}$ defined on an arbitrary family $\mathcal{K}$ of finite conditional random quantities, consider a finite subfamily $\mathcal{F}=\left\{X_{1}\left|H_{1}, \ldots, X_{n}\right| H_{n}\right\} \subseteq \mathcal{K}$ and the vector $\mathcal{M}=\left(\mu_{1}, \ldots, \mu_{n}\right)$, where $\mu_{i}=$ $\mathbb{P}\left(X_{i} \mid H_{i}\right)$ is the assessed prevision for the conditional random quantity $X_{i} \mid H_{i}, i \in\{1, \ldots, n\}$. With the pair $(\mathcal{F}, \mathcal{M})$ we associate the random gain $G=\sum_{i=1}^{n} s_{i} H_{i}\left(X_{i}-\mu_{i}\right)$ and we denote by $\mathcal{G}_{\mathcal{H}_{n}}$ the set of values of $G$ restricted to $\mathcal{H}_{n}=H_{1} \vee \cdots \vee H_{n}$. Then, by the betting scheme of de Finetti, coherence is defined as: 
Definition 1. The function $\mathbb{P}$ defined on $\mathcal{K}$ is coherent if and only if, $\forall n \geqslant 1, \forall s_{1}, \ldots, s_{n}, \forall \mathcal{F}=$ $\left\{X_{1}\left|H_{1}, \ldots, X_{n}\right| H_{n}\right\} \subseteq \mathcal{K}$, it holds that: $\min \mathcal{G}_{\mathcal{H}_{n}} \leqslant 0 \leqslant \max \mathcal{G}_{\mathcal{H}_{n}}$.

As it is well known, in Definition 1, the condition $\min \mathcal{G}_{\mathcal{H}_{n}} \leqslant 0 \leqslant \max \mathcal{G}_{\mathcal{H}_{n}}$ can be equivalently replaced by $\min \mathcal{G}_{\mathcal{H}_{n}} \leqslant 0$, or by $\max \mathcal{G}_{\mathcal{H}_{n}} \geqslant 0$.

A conditional prevision assessment $\mathbb{P}$ on $\mathcal{K}$ is not coherent, or incoherent, if and only if there exists a finite combination of $n$ bets such that $\min \mathcal{G}_{\mathcal{H}_{n}} \cdot \max \mathcal{G}_{\mathcal{H}_{n}}>0$, that is such that the values in $\mathcal{G}_{\mathcal{H}_{n}}$ are all positive, or all negative (Dutch Book). In other words, $\mathbb{P}$ is incoherent if and only if there exists a finite combination of $n$ bets such that, after discarding the case where all the bets are called off, the values of the random gain are all positive or all negative. In the particular case where $\mathcal{K}$ is a family of conditional events, then Definition 1 becomes the well known definition of coherence for a probability function, denoted as $P$, defined on $\mathcal{K}$.

By Definition 11, given any (finite) conditional random quantity $X \mid H$ and denoting by $x_{1}, \ldots, x_{r}$ the possible values of $X$ when $H$ is true, a prevision assessment $\mu$ on $X \mid H$ is coherent if and only if $\min \left\{x_{1}, \ldots, x_{r}\right\} \leqslant \mu \leqslant \max \left\{x_{1}, \ldots, x_{r}\right\}$. When $X$ is (the indicator of) an event $E$, with $P(E \mid H)=x$, the coherence of $x$ amounts to $0 \leqslant x \leqslant 1$, or $x=0$, or $x=1$, according to whether $\varnothing \neq E H \neq H$, or $E H=\varnothing$, or $E H=H$, respectively.

Given a family $\mathcal{F}=\left\{X_{1}\left|H_{1}, \ldots, X_{n}\right| H_{n}\right\}$, for each $i=1, \ldots, n$, we denote by $\left\{x_{i 1}, \ldots, x_{i r_{i}}\right\}$ the set of possible values for the restriction of $X_{i}$ to $H_{i}$; then, for each $i=1, \ldots, n$, and $j=1, \ldots, r_{i}$, we set $A_{i j}=\left(X_{i}=x_{i j}\right)$. Of course, for each $i$, the family $\left\{\bar{H}_{i}, A_{i j} H_{i}, j=1, \ldots, r_{i}\right\}$ is a partition of the sure event $\Omega$, with $A_{i j} H_{i}=A_{i j}$ and $\bigvee_{j=1}^{r_{i}} A_{i j}=H_{i}$, that is $A_{11} \vee \cdots \vee A_{1 r_{1}} \vee \bar{H}_{1}=\cdots=A_{n 1} \vee \cdots \vee A_{n r_{n}} \vee \bar{H}_{n}=\Omega$, or more explicitly $\left(X_{1}=x_{11}\right) \vee \cdots \vee\left(X_{1}=x_{1 r_{1}}\right) \vee \bar{H}_{1}=\cdots=\left(X_{n}=x_{n 1}\right) \vee \cdots \vee\left(X_{n}=x_{n r_{n}}\right) \vee \bar{H}_{n}=\Omega$. Then,

$$
\Omega=\left(A_{11} \vee \cdots \vee A_{1 r_{1}} \vee \bar{H}_{1}\right) \wedge \cdots \wedge\left(A_{n 1} \vee \cdots \vee A_{n r_{n}} \vee \bar{H}_{n}\right) .
$$

By expanding the expression in (1) and by discarding the logical conjunctions which coincide with $\varnothing$, we obtain a disjunctive representation of $\Omega$. The elements of this disjunction form a partition of $\Omega$ and are called the constituents generated by the family $\mathcal{F}$. We denote by $C_{1}, \ldots, C_{m}$ the constituents contained in $\mathcal{H}_{n}=H_{1} \vee \cdots \vee H_{n}$. Moreover, when $\mathcal{H}_{n} \neq \Omega$, we set $C_{0}=\overline{\mathcal{H}}_{n}=\bar{H}_{1} \cdots \bar{H}_{n}$. Hence $\Omega=\bigvee_{h=0}^{m} C_{h}$. In particular, the constituents generated by a family of $n$ conditional events $\left\{E_{1}\left|H_{1}, \ldots, E_{n}\right| H_{n}\right\}$ are obtained by expanding the expression $\left(E_{1} H_{1} \vee \bar{E}_{1} H_{1} \vee \bar{H}_{1}\right) \wedge \cdots \wedge\left(E_{n} H_{n} \vee \bar{E}_{n} H_{n} \vee \bar{H}_{n}\right)$, and by discarding the logical conjunctions which are impossible. If $E_{1}, \ldots, E_{n}, H_{1}, \ldots, H_{n}$ are logically independent, then the number of constituents for the family $\left\{E_{1}\left|H_{1}, \ldots, E_{n}\right| H_{n}\right\}$ is $3^{n}$ (in which case the conditional events $E_{1}\left|H_{1}, \ldots, E_{n}\right| H_{n}$ are said logically independent). Given a prevision assessment $\mathcal{M}=\left(\mu_{1}, \ldots, \mu_{n}\right)$ on $\mathcal{F}=\left\{X_{1}\left|H_{1}, \ldots, X_{n}\right| H_{n}\right\}$, with each constituent $C_{h}, h=1, \ldots, m$, we associate a vector

$$
Q_{h}=\left(q_{h 1}, \ldots, q_{h n}\right), \text { with } q_{h i}=\left\{\begin{array}{l}
x_{i j}, \text { if } C_{h} \subseteq A_{i j}, j=1, \ldots, r_{i}, \\
\mu_{i}, \text { if } C_{h} \subseteq \bar{H}_{i} .
\end{array}\right.
$$

With $C_{0}$ it is associated $Q_{0}=\mathcal{M}=\left(\mu_{1}, \ldots, \mu_{n}\right)$. As, for each $i, j$, the quantities $x_{i j}, \mu_{i}$ are real numbers, it holds that $Q_{h} \in \mathbb{R}^{n}, h=0,1, \ldots, m$. Denoting by $\mathcal{I}$ the convex hull of $Q_{1}, \ldots, Q_{m}$, the condition $\mathcal{M} \in \mathcal{I}$ amounts to the existence of a vector $\left(\lambda_{1}, \ldots, \lambda_{m}\right)$ such that: $\sum_{h=1}^{m} \lambda_{h} Q_{h}=\mathcal{M}, \sum_{h=1}^{m} \lambda_{h}=1, \lambda_{h} \geqslant 0, \forall h$; in other words, $\mathcal{M} \in \mathcal{I}$ is equivalent to the solvability of the system $(\Sigma)$ given below.

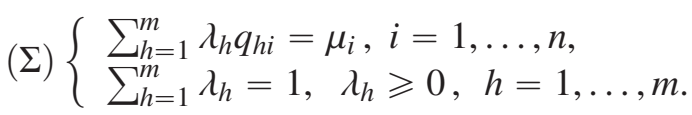

We say that $(\Sigma)$ is the system associated to the pair $(\mathcal{F}, \mathcal{M})$. By a suitable alternative theorem, it can be shown that the solvability of $(\Sigma)$ is equivalent to the condition: $\min \mathcal{G}_{\mathcal{H}_{n}} \leqslant 0 \leqslant \max \mathcal{G}_{\mathcal{H}_{n}}$. 
Let $\mathbf{x}=\left(x_{1}, \ldots, x_{m}\right), \mathbf{y}=\left(y_{1}, \ldots, y_{n}\right)^{t}$ and $A=\left(a_{h i}\right)$ be, respectively, a row $m$-vector, a column $n$-vector and a $m \times n$-matrix. The vector $\mathbf{x}$ is said semipositive if $x_{h} \geqslant 0, h=1, \ldots, m$, and $x_{1}+\cdots+x_{m}>0$. Then, we have (cf. [23], Theorem 2.9)

Theorem 1. Exactly one of the following alternatives holds: (i) the equation $\mathbf{x} A=0$ has a semipositive solution; (ii) the inequality $A \mathbf{y}>0$ has a solution.

We observe that, choosing $a_{h i}=q_{h i}-\mu_{i}, h=1, \ldots, m, i=1, \ldots, n$, the solvability of $\mathbf{x} A=0$ means that $\mathcal{M} \in \mathcal{I}$, while the solvability of $A \mathbf{y}>0$ means that, choosing $s_{i}=y_{i}, i=1, \ldots, n$, one has $\min \mathcal{G}_{\mathcal{H}_{n}}>0$ (and hence $\mathcal{M}$ would be incoherent). Therefore, by applying Theorem 1 with $A=\left(q_{h i}-\mu_{i}\right)$, we obtain that $\mathcal{M} \in \mathcal{I}$ if and only if $\min \mathcal{G}_{\mathcal{H}_{n}} \leqslant 0$, that is, $(\Sigma)$ is solvable if and only if $\min \mathcal{G}_{\mathcal{H}_{n}} \leqslant 0$.

Given a nonempty subset $J \subseteq\{1, \ldots, n\}$, we set $\mathcal{F}_{J}=\left\{X_{j} \mid H_{j}: j \in J\right\}$ and $\mathcal{M}_{J}=\left(\mu_{j}: j \in J\right)$, then we denote by $\left(\Sigma_{J}\right)$ the system associated to the pair $\left(\mathcal{F}_{J}, \mathcal{M}_{J}\right)$. Of course, when $J=\{1, \ldots, n\}$ it holds that $\left(\mathcal{F}_{J}, \mathcal{M}_{J}\right)=(\mathcal{F}, \mathcal{M})$ and $\left(\Sigma_{J}\right)=(\Sigma)$. Then, by Definition 1 and Theorem 1 , for the prevision assessment $\mathcal{M}$ on $\mathcal{F}$ it holds that

$$
\mathcal{M} \text { is coherent } \Longleftrightarrow\left(\Sigma_{J}\right) \text { is solvable }, \forall J \subseteq\{1, \ldots, n\} .
$$

In other words $\mathcal{M}$ on $\mathcal{F}$ is coherent if and only if, for every nonempty subset $J \subseteq\{1, \ldots, n\}$, the sub-vector $\mathcal{M}_{J}$ belongs to the convex hull $\mathcal{I}_{J}$ associated to the pair $\left(\mathcal{F}_{J}, \mathcal{M}_{J}\right)$.

Given the assessment $\mathcal{M}=\left(\mu_{1}, \ldots, \mu_{n}\right)$ on $\mathcal{F}=\left\{X_{1}\left|H_{1}, \ldots, X_{n}\right| H_{n}\right\}$, let $S$ be the set of solutions $\Lambda=\left(\lambda_{1}, \ldots, \lambda_{m}\right)$ of system $(\Sigma)$ defined in (3). We point out that the solvability of system $(\Sigma)$ (i.e., the condition $\mathcal{M} \in \mathcal{I}$ ) is a necessary (but not sufficient) condition for coherence of $\mathcal{M}$ on $\mathcal{F}$. We introduce, for each $i=1, \ldots, n$, the function $\sum_{h: C_{h} \subseteq H_{i}} \lambda_{h}$ of the vector $\Lambda=\left(\lambda_{1}, \ldots, \lambda_{m}\right)$. Moreover, by assuming the system $(\Sigma)$ solvable, that is $S \neq \varnothing$, we compute the maximum $M_{i}$ of the function $\sum_{h: C_{h} \subseteq H_{i}} \lambda_{h}$ with respect to $\Lambda \in S$. Then, we define:

$$
I_{0}=\left\{i: M_{i}=0 ; i=1, \ldots, n\right\}, \quad \mathcal{F}_{0}=\left\{X_{i} \mid H_{i}, i \in I_{0}\right\}, \quad \mathcal{M}_{0}=\left(\mu_{i}, i \in I_{0}\right) .
$$

We observe that $i \in I_{0}$ if and only if the (unique) coherent extension of $\mathcal{M}$ to $H_{i} \mid \mathcal{H}_{n}$ is zero.

Of course, the previous notions can be used in the case of conditional events. We observe that, given a probability assessment $\mathcal{P}=\left(p_{1}, \ldots, p_{n}\right)$ on a family of $n$ conditional events $\mathcal{F}=\left\{E_{1}\left|H_{1}, \ldots, E_{n}\right| H_{n}\right\}$, we can determine the constituents $C_{0}, C_{1}, \ldots, C_{m}$, where $C_{0}=\bar{H}_{1} \cdots \bar{H}_{n}$, and the associated points $Q_{0}, Q_{1}, \ldots, Q_{m}$, where $Q_{0}=\mathcal{P}$. We observe that $Q_{h}=\left(q_{h 1}, \ldots, q_{h n}\right)$, with $q_{h i} \in\left\{1,0, p_{i}\right\}, i=1, \ldots, n$. We also observe that given a subset $J \subset\{1, \ldots, n\}$, we can determine the constituents $C_{h J}$ 's and the corresponding points $Q_{h J}$ 's associated to the pair $\left(\mathcal{F}_{J}, \mathcal{M}_{J}\right)$. We set $J^{c}=\{1, \ldots, n\} \backslash J$ and if, for instance, $J=\{1, \ldots, r\}$, with $r<n$, then $J^{c}=\{r+1, \ldots, n\}$ and $\mathcal{P}=\left(\mathcal{P}_{J}, \mathcal{P}_{J^{c}}\right)$. Moreover, each point $Q_{h}$ can be represented, for suitable indexes $i_{h}, k_{h}$, as $Q_{h}=\left(Q_{i_{h} J}, Q_{k_{h} J^{c}}\right)$; then, any linear convex combination $\sum_{h} \lambda_{h} Q_{h}$ coincides with $\left(\sum_{h} \lambda_{h} Q_{i_{h} J}, \sum_{h} \lambda_{h} Q_{k_{h} J^{c}}\right)$. A similar representation holds for $J=\left\{i_{1}, \ldots, i_{r}\right\}$, after a suitable permutation of indexes. On this basis, we recall three results ([24, Theorems 3.1, 3.2, 3.3]).

Theorem 2. Given a subset $J \subset\{1, \ldots, n\}$, if there exist $m$ nonnegative coefficients $\lambda_{1}, \ldots, \lambda_{m}$, with $\sum_{h=1}^{m} \lambda_{h}=1$, such that $\mathcal{P}_{J}=\sum_{h=1}^{m} \lambda_{h} Q_{i_{h} J}$, if $\sum_{h: C_{h} \subseteq H_{J}} \lambda_{h}>0$, where $H_{J}=\bigvee_{j \in J} H_{j}$, then $\mathcal{P}_{J} \in \mathcal{I}_{J}$

Theorem 3. If $\mathcal{P} \in \mathcal{I}$, then for every $J \subset\{1, \ldots, n\}$ such that $J \backslash I_{0} \neq \varnothing$ it holds that $\mathcal{P}_{J} \in \mathcal{I}_{J}$.

Theorem 4. The conditional probability assessment $\mathcal{P}=\left(p_{1}, \ldots, p_{n}\right)$ on the family $\mathcal{F}=$ $\left\{E_{1}\left|H_{1}, \ldots, E_{n}\right| H_{n}\right\}$ is coherent if and only if the following conditions are satisfied:

(i) $\mathcal{P} \in \mathcal{I}$; (ii) if $I_{0} \neq \varnothing$, then $\mathcal{P}_{0}$ is coherent. 
We remark that when we consider prevision assessments on conditional random quantities results similar to Theorems 2, 3, 4 can be obtained. In particular, by taking into account that $\mathcal{M} \in \mathcal{I}$ amounts to the solvability of system $(\Sigma)$, Theorem 4 becomes

Theorem 5. The conditional prevision assessment $\mathcal{M}=\left(\mu_{1}, \ldots, \mu_{n}\right)$ on the family $\mathcal{F}=$ $\left\{X_{1}\left|H_{1}, \ldots, X_{n}\right| H_{n}\right\}$ is coherent if and only if the following conditions are satisfied:

(i) the system $(\Sigma)$ in (3) is solvable; (ii) if $I_{0} \neq \varnothing$, then $\mathcal{M}_{0}$ is coherent.

We observe that, when $I_{0}=\varnothing$, coherence of $\mathcal{M}$ amounts to solvability of $(\Sigma)$. In order to illustrate the previous results, we examine two examples.

Example 1. Let $A, H, K$ be three events, with $A, H, K$ logically independent. Moreover, let $\mathcal{P}=(x, y)$ be a probability assessment on the family $\mathcal{E}=\{A|H, A| K\}$, where $x=P(A \mid H), y=P(A \mid K)$. The constituents generated by $\mathcal{E}$ and contained in $H \vee K$ are: $C_{1}=A H K, C_{2}=\bar{A} H K, C_{3}=\bar{A} \bar{H} K, C_{4}=\bar{A} H \bar{K}, C_{5}=A \bar{H} K$, $C_{6}=A H \bar{K}$. Then, the points $Q_{h}$ 's associated with $C_{1}, \ldots, C_{6}$ are: $Q_{1}=(1,1), Q_{2}=(0,0), Q_{3}=(x, 0)$, $Q_{4}=(0, y), Q_{5}=(x, 1), Q_{6}=(1, y)$. Moreover $C_{0}=\bar{H} \bar{K}$ and $Q_{0}=(x, y)=\mathcal{P}$. The condition $\mathcal{P} \in \mathcal{I}$, where $\mathcal{I}$ is the convex hull of the points $Q_{1}, \ldots, Q_{6}$, amounts to the solvability of the system $(\Sigma)$ below

$$
\lambda_{1}+\lambda_{3} x+\lambda_{5} x+\lambda_{6}=x, \lambda_{1}+\lambda_{4} y+\lambda_{5}+\lambda_{6} y=y, \lambda_{1}+\cdots+\lambda_{6}=1, \quad \lambda_{h} \geqslant 0, \quad \forall h .
$$

We observe that, for each $(x, y) \in[0,1]^{2}$, the vector $\Lambda=\left(\lambda_{1}, \ldots, \lambda_{6}\right)=\left(0,0, \frac{1-y}{2}, \frac{1-x}{2}, \frac{y}{2}, \frac{x}{2}\right)$ is a solution of $(\Sigma)$; indeed $\mathcal{P}=\lambda_{1} Q_{1}+\cdots+\lambda_{6} Q_{6}=\frac{1-y}{2} Q_{3}+\frac{1-x}{2} Q_{4}+\frac{y}{2} Q_{5}+\frac{x}{2} Q_{6}=\frac{1-y}{2}(x, 0)+\frac{1-x}{2}(0, y)+$ $\frac{y}{2}(x, 1)+\frac{x}{2}(1, y)=(x, y)$. Moreover, for this solution it holds that $\sum_{C_{h} \subseteq H} \lambda_{h}=\lambda_{1}+\lambda_{2}+\lambda_{4}+\lambda_{6}=\frac{1}{2}>0$ and $\sum_{C_{h} \subseteq K} \lambda_{h}=\lambda_{1}+\lambda_{2}+\lambda_{3}+\lambda_{5}=\frac{1}{2}>0$. Then, $I_{0}=\varnothing$ and by Theorem 5 the assessment $(x, y)$ is coherent, for every $(x, y) \in[0,1]^{2}$. Notice that in particular cases, like $x=0$ or $x=1$, the number of distinct points $Q_{h}$ 's is less than 6, anyway the previous analysis is still valid. For instance, when $x=0$ and $0<y<1$ it holds that $Q_{2}=Q_{3}, \Lambda=\left(\lambda_{1}, \ldots, \lambda_{6}\right)=\left(0,0, \frac{1-y}{2}, \frac{1}{2}, \frac{y}{2}, 0\right)$, and in geometrical terms it holds that $\mathcal{P}=\frac{1-y}{2} Q_{3}+\frac{1}{2} Q_{4}+\frac{y}{2} Q_{5}=\frac{1-y}{2}(0,0)+\frac{1}{2}(0, y)+\frac{y}{2}(0,1)=(0, y)$.

Example 2. Let $A, H, K$ be three events, with $H K=\varnothing$ and $A$ logically independent from $H$ and $K$. Moreover, let $\mathcal{P}=(x, y)$ be a probability assessment on the family $\mathcal{E}=\{A|H, A| K\}$. The constituents generated by $\mathcal{E}$ are $C_{1}=\bar{A} \bar{H} K, C_{2}=\bar{A} H \bar{K}, C_{3}=A \bar{H} K, C_{4}=A H \bar{K}, C_{0}=\bar{H} \bar{K}$ (which coincide with $C_{3}, C_{4}, C_{5}, C_{6}, C_{0}$ examined in the Example 11 respectively). The associated points $Q_{h}$ 's are $Q_{1}=$ $(x, 0), Q_{2}=(0, y), Q_{3}=(x, 1), Q_{4}=(1, y), \mathcal{P}=(x, y)$. We observe that, for each $(x, y) \in[0,1]^{2}$, the vector $\left(\lambda_{1}, \ldots, \lambda_{4}\right)=\left(\frac{1-y}{2}, \frac{1-x}{2}, \frac{y}{2}, \frac{x}{2}\right)$ is a solution of $(\Sigma)$, with $I_{0}=\varnothing$. Then, by Theorem 5 the assessment $(x, y)$ is coherent, for every $(x, y) \in[0,1]^{2}$.

We recall the following extension theorem for conditional previsions, which is a generalization of de Finetti's fundamental theorem of probability to conditional random quantities (see, e.g.,[37, 50, 54])

Theorem 6. Let $\mathcal{M}=\left(\mu_{1}, \ldots, \mu_{n}\right)$ be a coherent prevision assessment on a family of bounded conditional random quantities $\mathcal{F}=\left\{X_{1}\left|H_{1}, \ldots, X_{n}\right| H_{n}\right\}$. Moreover, let $X \mid H$ be a further bounded conditional random quantity. Then, there exists a suitable closed interval $\left[\mu^{\prime}, \mu^{\prime \prime}\right]$ such that the extension $\mu=\mathbb{P}(X \mid H)$ is coherent if and only if $\mu \in\left[\mu^{\prime}, \mu^{\prime \prime}\right]$.

\subsection{A deepening on conditional random quantities}

The indicator of a conditional event $E \mid H$ (denoted by the same symbol), with $P(E \mid H)=x$, is defined as 


$$
E \mid H=E H+x \bar{H}=E H+x(1-H)= \begin{cases}1, & \text { if } E H \text { is true, } \\ 0, & \text { if } \bar{E} H \text { is true, } \\ x, & \text { if } \bar{H} \text { is true. }\end{cases}
$$

Of course, the third value of the random quantity $E \mid H$ (subjectively) depends on the assessed probability $P(E \mid H)=x$. Notice that, when $H \subseteq E$ (i.e., $E H=H$ ), by coherence $P(E \mid H)=1$ and hence for the indicator it holds that $E \mid H=H+P(E \mid H) \bar{H}=1$. Moreover, when $E H=\varnothing$, by coherence $P(E \mid H)=0$ and hence $E \mid H=E H+P(E \mid H) \bar{H}=0$. The negation of a conditional event $E \mid H$ is defined as $\overline{E \mid H}=$ $\bar{E}|H=1-E| H$. We recall that, in the subjective approach to probability, if you assess $\mathbb{P}(X \mid H)=\mu$, then you agree to pay $\mu$ by knowing that you will receive the amount $X H+\mu \bar{H}$, which coincides with $X$, if $H$ is true, or with $\mu$, if $H$ is false (bet called off). Usually, in literature the conditional random quantity $X \mid H$ is defined as the restriction of $X$ to $H$, which coincides with $X$, when $H$ is true, and it is undefined when $H$ is false. Under this point of view, (when $H$ is false) $X \mid H$ does not coincide with $X H+\mu \bar{H}$. However, by coherence, it holds that

$$
\mathbb{P}(X H+\mu \bar{H})=\mathbb{P}(X H)+\mu P(\bar{H})=\mathbb{P}(X \mid H) P(H)+\mu P(\bar{H})=\mu P(H)+\mu P(\bar{H})=\mu .
$$

Therefore, once a coherent assessment $\mu=\mathbb{P}(X \mid H)$ is specified, we can extend the notion of $X \mid H$, by defining its value as equal to $\mu$ when $H$ is false (for further details see [30]). Then, denoting by $x_{1}, \ldots, x_{r}$ the possible values of $X$ when $H$ is true, it holds that

$$
X \mid H=X H+\mu \bar{H} \in\left\{x_{1}, \ldots, x_{r}, \mu\right\} .
$$

By (7) the prevision of the extended notion of $X \mid H$, as defined in (8), coincides with the conditional prevision $\mu=\mathbb{P}(X \mid H)$ where $X \mid H$ is looked at as the restriction of $X$ to $H$. From $(8) X \mid H$ can be interpreted as the amount that you receive when you pay its prevision $\mu$. Then, the random gain $G$ can be also represented as $G=s(X \mid H-\mu)$. In particular, when $X$ is (the indicator of) an event $E$, we obtain $E \mid H=E H+P(E \mid H) \bar{H}$, that is formula (6). Moreover, the prevision $\mathbb{P}(E \mid H)$ of (the conditional random quantity) $E \mid H$ coincides with the conditional probability $P(E \mid H)$. For related discussions, see also [11, 28, 42].

Remark 1. Given a prevision assessment $\mathcal{M}=\left(\mu_{1}, \ldots, \mu_{n}\right)$ on a family of $n$ conditional random quantities $\left\{X_{1}\left|H_{1}, \ldots, X_{n}\right| H_{n}\right\}$, based on (8) we observe that for each constituent $C_{h}$ the corresponding point $Q_{h}$ represents the value assumed by the random vector $\left(X_{1}\left|H_{1}, \ldots, X_{n}\right| H_{n}\right)$ when $C_{h}$ is true. In particular, when $C_{0}$ is true the value of the random vector is the prevision point $\mathcal{M}$.

\subsection{Conjunction and disjunction of conditional events}

We recall now the notion of conjoined conditional which was introduced in the framework of conditional random quantities $([28,29,30,33])$. Given a coherent probability assessment $(x, y)$ on $\{A|H, B| K\}$ we consider the random quantity $A H B K+x \bar{H} B K+y \bar{K} A H$ and we set $\mathbb{P}[(A H B K+x \bar{H} B K+y \bar{K} A H) \mid(H \vee K)]=z$. Then we define the conjunction $(A \mid H) \wedge(B \mid K)$ as follows:

Definition 2. Given a coherent prevision assessment $P(A \mid H)=x, P(B \mid K)=y$, and $\mathbb{P}[(A H B K+x \bar{H} B K+$ $y \bar{K} A H) \mid(H \vee K)]=z$, the conjunction $(A \mid H) \wedge(B \mid K)$ is the conditional random quantity defined as

$$
\begin{aligned}
& (A \mid H) \wedge(B \mid K)=(A H B K+x \bar{H} B K+y \bar{K} A H) \mid(H \vee K)= \\
& =(A H B K+x \bar{H} B K+y \bar{K} A H)(H \vee K)+z \bar{H} \bar{K}= \begin{cases}1, & \text { if } A H B K \text { is true, } \\
0, & \text { if } \bar{A} H \vee \bar{B} K \text { is true, } \\
x, & \text { if } \bar{H} B K \text { is true, } \\
y, & \text { if } A H \bar{K} \text { is true, } \\
z, & \text { if } \bar{H} \bar{K} \text { is true. }\end{cases}
\end{aligned}
$$


Of course, by recalling (7), it holds that $\mathbb{P}[(A \mid H) \wedge(B \mid K)]=z$. Notice that in (9) the conjunction is represented as $X \mid H$ is in (8) and, once the (coherent) assessment $(x, y, z)$ is given, the conjunction $(A \mid H) \wedge$ $(B \mid K)$ is (subjectively) determined. Conversely, each given conjunction uniquely determines a coherent assessment $(x, y, z)$. We recall that, in betting terms, $z$ represents the amount you agree to pay, with the proviso that you will receive the quantity $(A \mid H) \wedge(B \mid K)=A H B K+x \bar{H} B K+y \bar{K} A H+z \bar{H} \bar{K}$, which assumes one of the following values: 1 , if both conditional events are true; 0 , if at least one of the conditional events is false; the probability of the conditional event that is void, if one conditional event is void and the other one is true; the payed amount $z$, if both conditional events are void. We recall that $A|H=B| K$ amounts to $A H=B K$ and $H=K$. Thus, when $A|H=B| K$ it holds that $(A H B K+x \bar{H} B K+y A H \bar{K}) \mid(H \vee$ $K)=A H|H=A| H$, that is $(A \mid H) \wedge(A \mid H)=A \mid H$. Moreover the conjunction is commutative, that is $(A \mid H) \wedge(B \mid K)=(B \mid K) \wedge(A \mid H)$. The next result shows that the Fréchet-Hoeffding bounds still hold for the conjunction of two conditional events ([30, Theorem 7]).

Theorem 7. Given any coherent assessment $(x, y)$ on $\{A|H, B| K\}$, with $A, H, B, K$ logically independent, and with $H \neq \varnothing, K \neq \varnothing$, the extension $z=\mathbb{P}[(A \mid H) \wedge(B \mid K)]$ is coherent if and only if the following Fréchet-Hoeffding bounds are satisfied:

$$
\max \{x+y-1,0\}=z^{\prime} \leqslant z \leqslant z^{\prime \prime}=\min \{x, y\} .
$$

From Definition 2 and Theorem 7 it holds that

$$
\max \{A|H+B| K-1,0\} \leqslant(A \mid H) \wedge(B \mid K) \leqslant \min \{A|H, B| K\} .
$$

Remark 2. We observe that, by logical independence, the assessment $(x, y)$ on $\{A|H, B| K\}$ is coherent for every $(x, y) \in[0,1]^{2}$. Then, from Theorems 6 and 7 the set $\Pi$ of coherent prevision assessments $(x, y, z)$ on $\{A|H, B| K,(A \mid H) \wedge(B \mid K)\}$ is

$$
\Pi=\left\{(x, y, z):(x, y) \in[0,1]^{2}, \max \{x+y-1,0\} \leqslant z \leqslant \min \{x, y\}\right\} .
$$

The set $\Pi$ is the tetrahedron with vertices the points $(1,1,1),(1,0,0),(0,1,0),(0,0,0)$. Notice that, the assumption of logical independence plays a key role for the validity of Theorem 7 . Indeed, in case of some logical dependencies, for the interval $\left[z^{\prime}, z^{\prime \prime}\right]$ of coherent extensions $z$ it holds that $\max \{x+y-1,0\} \leqslant$ $z^{\prime} \leqslant z^{\prime \prime} \leqslant \min \{x, y\}$. For instance, when $H=K$ and $A B=\varnothing$, the coherence of the assessment $(x, y)$ on $\{A|H, B| H\}$ is equivalent to the condition $x+y-1 \leqslant 0$. In this case, it holds that $(A \mid H) \wedge(B \mid H)=A B \mid H$ with $P(A B \mid H)=0$; then, the unique coherent extension on $A B \mid H$ is $z=0$. As another example, in the case $A=B$, with $A, H, K$ logically independent, it holds that the assessment $(x, y)$ on $\{A|H, A| K\}$ is coherent for every $(x, y) \in[0,1]^{2}$. Moreover, as it will be shown by Theorem 17, the extension $z$ is coherent if and only if $x y \leqslant z \leqslant \min \{x, y\}$. Finally, we remark that in all cases, for each coherent extension $z$, it holds that $z \in\left[z^{\prime}, z^{\prime \prime}\right] \subseteq[0,1]$; thus $(A \mid H) \wedge(B \mid K) \in[0,1]$.

Other approaches to compounded conditionals, which are not based on coherence, can be found in [6, 22, 39, 44]. A study of the lower and upper bounds for other definitions of conjunction, where the conjunction is a conditional event like Adams' quasi conjunction, has been given in [51].

We recall now the notion of disjoined conditional. Given a coherent probability assessment $(x, y)$ on $\{A|H, B| K\}$ we consider the random quantity $(A H \vee B K)+x \bar{H} \bar{B} K+y \bar{K} \bar{A} H$ and we set $\mathbb{P}[((A H \vee B K)+$ $x \bar{H} \bar{B} K+y \bar{K} \bar{A} H) \mid(H \vee K)]=w$. Then we define the disjunction $(A \mid H) \vee(B \mid K)$ as follows: 
Definition 3. Given a coherent prevision assessment $P(A \mid H)=x, P(B \mid K)=y$, and $\mathbb{P}[((A H \vee B K)+$ $x \bar{H} \bar{B} K+y \bar{K} \bar{A} H) \mid(H \vee K)]=w$, the disjunction $(A \mid H) \vee(B \mid K)$ is the conditional random quantity

$$
\begin{aligned}
& (A \mid H) \vee(B \mid K)=((A H \vee B K)+x \bar{H} \bar{B} K+y \bar{K} \bar{A} H) \mid(H \vee K)= \\
& =((A H \vee B K)+x \bar{H} \bar{B} K+y \bar{K} \bar{A} H)(H \vee K)+w \bar{H} \bar{K}= \begin{cases}1, & \text { if } A H \vee B K \text { is true, } \\
0, & \text { if } \bar{A} H \bar{B} K \text { is true, } \\
x, & \text { if } \bar{H} \bar{B} K \text { is true, } \\
y, & \text { if } \bar{A} H \bar{K} \text { is true, } \\
w, & \text { if } \bar{H} \bar{K} \text { is true. }\end{cases}
\end{aligned}
$$

We recall the notion of conjunction of $n$ conditional events ([33]).

Definition 4. Let $n$ conditional events $E_{1}\left|H_{1}, \ldots, E_{n}\right| H_{n}$ be given. For each non-empty strict subset $S$ of $\{1, \ldots, n\}$, let $x_{S}$ be a prevision assessment on $\bigwedge_{i \in S}\left(E_{i} \mid H_{i}\right)$. Then, the conjunction $\left(E_{1} \mid H_{1}\right) \wedge \cdots \wedge\left(E_{n} \mid H_{n}\right)$ is the conditional random quantity $\mathcal{C}_{1 \ldots n}$ defined as

$$
\begin{aligned}
& \mathcal{C}_{1 \cdots n}=\left[\bigwedge_{i=1}^{n} E_{i} H_{i}+\sum_{\varnothing \neq S \subset\{1,2 \ldots, n\}} x_{S}\left(\bigwedge_{i \in S} \bar{H}_{i}\right) \wedge\left(\bigwedge_{i \notin S} E_{i} H_{i}\right)\right] \mid\left(\bigvee_{i=1}^{n} H_{i}\right)= \\
& = \begin{cases}1, & \text { if } \bigwedge_{i=1}^{n} E_{i} H_{i} \text { is true, } \\
0, & \text { if } \bigvee_{i=1}^{n} \bar{E}_{i} H_{i} \text { is true, } \\
x_{S}, & \text { if }\left(\bigwedge_{i \in S} \bar{H}_{i}\right) \wedge\left(\bigwedge_{i \notin S} E_{i} H_{i}\right) \text { is true, } \varnothing \neq S \subset\{1,2 \ldots, n\}, \\
x_{1 \ldots n}, & \text { if } \bigwedge_{i=1}^{n} \bar{H}_{i} \text { is true, }\end{cases}
\end{aligned}
$$

where

$$
x_{1 \cdots n}=x_{\{1, \ldots, n\}}=\mathbb{P}\left(\mathcal{C}_{1 \cdots n}\right)=\mathbb{P}\left[\left(\bigwedge_{i=1}^{n} E_{i} H_{i}+\sum_{\varnothing \neq S \subset\{1,2 \ldots, n\}} x_{S}\left(\bigwedge_{i \in S} \bar{H}_{i}\right) \wedge\left(\bigwedge_{i \notin S} E_{i} H_{i}\right)\right) \mid\left(\bigvee_{i=1}^{n} H_{i}\right)\right]
$$

Of course, we obtain $\mathcal{C}_{1}=E_{1} \mid H_{1}$, when $n=1$. In Definition 4 each possible value $x_{S}$ of $\mathcal{C}_{1 \cdots n}, \varnothing \neq S \subset$ $\{1, \ldots, n\}$, is evaluated when defining (in a previous step) the conjunction $\mathcal{C}_{S}=\bigwedge_{i \in S}\left(E_{i} \mid H_{i}\right)$. Then, after the conditional prevision $x_{1 \ldots n}$ is evaluated, $\mathcal{C}_{1 \ldots n}$ is completely specified. Of course, we require coherence for the prevision assessment $\left(x_{S}, \varnothing \neq S \subseteq\{1, \ldots, n\}\right)$, so that $\mathcal{C}_{1 \cdots n} \in[0,1]$. In the framework of the betting scheme, $x_{1 \ldots n}$ is the amount that you agree to pay with the proviso that you will receive:

- 1 , if all conditional events are true;

- 0 , if at least one of the conditional events is false;

- the prevision of the conjunction of that conditional events which are void, otherwise. In particular you receive back $x_{1 \ldots n}$ when all conditional events are void.

The operation of conjunction is associative and commutative ([33, Proposition 1]). We recall below a necessary condition of coherence related with the Fréchet-Hoeffding bounds ([33, Theorem 13]).

Theorem 8. Let $\left(x_{1}, \ldots, x_{n}, x_{1 \ldots n}\right)$ be a coherent prevision assessment on the family $\left\{E_{1}\left|H_{1}, \ldots, E_{n}\right| H_{n}, \mathcal{C}_{1 \ldots n}\right\}$. Then, $\max \left\{\sum_{i=1}^{n} x_{i}-(n-1), 0\right\} \leqslant x_{1 \cdots n} \leqslant \min \left\{x_{1}, \ldots, x_{n}\right\}$.

\subsection{Frank t-norms}

We recall below the notion of t-norm (see [36, 41, 40]).

Definition 5. A t-norm is a function $T:[0,1]^{2} \longrightarrow[0,1]$ which satisfies, for all $x, y, z \in[0,1]$, the following four axioms: $T(x, y)=T(y, x)$ (commutativity); $T(x, T(y, z))=T(T(x, y), z)$ (associativity); $T(x, y) \leqslant T(x, z)$ whenever $y \leqslant z$ (monotonicity); $T(x, 1)=x$ (boundary condition). 
Some basic t-norms are the minimum $T_{M}$ (which is the greatest t-norm), the product $T_{P}$, the Eukasiewicz t-norm $T_{L}$, given below:

$$
T_{M}(x, y)=\min (x, y), \quad T_{P}(x, y)=x y, \quad T_{L}(x, y)=\max (x+y-1,0) .
$$

Frank t-norms are a relevant class of t-norms to which the previous basic ones belong. The Frank t-norm $T_{\lambda}:[0,1]^{2} \rightarrow[0,1]$, with parameter $\lambda \in[0,+\infty]$, is defined as

$$
T_{\lambda}(x, y)= \begin{cases}T_{M}(x, y)=\min \{x, y\}, & \text { if } \lambda=0, \\ T_{P}(x, y)=x y, & \text { if } \lambda=1, \\ T_{L}(x, y)=\max \{x+y-1,0\}, & \text { if } \lambda=+\infty, \\ \log _{\lambda}\left(1+\frac{\left(\lambda^{x}-1\right)\left(\lambda^{y}-1\right)}{\lambda-1}\right), & \text { otherwise. }\end{cases}
$$

We recall that $T_{\lambda}$ is continuous with respect to $\lambda$; moreover, it is decreasing with respect to the parameter $\lambda$. Then, for each given $(x, y) \in[0,1]^{2}$, it holds that $T_{L}(x, y) \leqslant T_{\lambda}(x, y) \leqslant T_{M}(x, y)$, for every $\lambda \in[0,+\infty]$ (see, e.g., [41], [40]). Frank t-norms provide a gradual transition between Lukasiewicz t-norm $(\lambda=+\infty)$ and minimum t-norm $(\lambda=0)$. Frank t-norms have been exploited in [8] (see also [13]) with the aim of obtaining the coherent values for the membership function of the intersection of two fuzzy subsets. Since t-norms are associative they can be extended in a unique way to an $n$-ary operation for arbitrary integer $n \geqslant 2$ (see [36, 40]).

\section{Sharpness of the Fréchet-Hoeffding bounds for the conjunction of $\boldsymbol{n}$ conditional events}

In this section we show, under logical independence, the sharpness of Fréchet-Hoeffding bounds for the prevision of the conjunction $\mathcal{C}_{1 \ldots n}$ and we illustrate some details by considering the case $n=3$. We also show that the set of all coherent prevision assessments on $\mathcal{F}=\left\{E_{1}\left|H_{1}, \ldots, E_{n}\right| H_{n}, \mathcal{C}_{1 \ldots n}\right\}$ is convex.

Let $\mathcal{M}=\left(x_{1}, \ldots, x_{n}, x_{1 \ldots n}\right)$ be a prevision assessment on $\mathcal{F}=\left\{E_{1}\left|H_{1}, \ldots, E_{n}\right| H_{n}, \mathcal{C}_{1 \ldots n}\right\}$, with $E_{1}, \ldots, E_{n}, H_{1}, \ldots, H_{n}$ logically independent. In order to determine the constituents generated by the family $\mathcal{F}$ it is enough to consider the constituents $C_{0}, C_{1}, \ldots, C_{m}$ generated by the family $\left\{E_{1}\left|H_{1}, \ldots, E_{n}\right| H_{n}\right\}$, where by logical independence $m+1=3^{n}$. Indeed, each $C_{h}$ uniquely determines the value of $\mathcal{C}_{1 \ldots n}$, that is for each $h$ there exists a unique $x_{S}$ such that $C_{h}$ logically implies the event $\left(\mathcal{C}_{1 \cdots n}=x_{S}\right)$ and hence $C_{h} \wedge\left(\mathcal{C}_{1 \cdots n}=x_{S}\right)=C_{h}$. Then, $C_{0}, C_{1}, \ldots, C_{m}$ also represent the constituents generated by the family $\mathcal{F}$. By Remark 1 , for each $C_{h}$ the associated point $Q_{h}$ represents the value of the random vector $\left(E_{1}\left|H_{1}, \ldots, E_{n}\right| H_{n}, \mathrm{C}_{1 \cdots n}\right)$ when $C_{h}$ is true. The last component of $Q_{h}$ is the value of $\mathcal{C}_{1 \cdots n}$ when $C_{h}$ is true. By Definition 4, we observe that, when the conditioning events $H_{1}, \ldots, H_{n}$ are all true, it holds that $\mathcal{C}_{1 \ldots n} \in\{1,0\}$. In this section we only need to consider the constituents $C_{h}$ 's such that $C_{h} \subseteq \bigwedge_{i=1}^{n} H_{i}$. For these constituents the associated $Q_{h}^{\prime}$ s have binary components, where the last component is 1 , or 0 , according to whether $C_{h}=\bigwedge_{i=1}^{n} E_{i} H_{i}$, or $C_{h} \subseteq\left(\bigvee_{i=1}^{n} \bar{E}_{i}\right) \wedge\left(\bigwedge_{i=1}^{n} H_{i}\right)$. Given any subset $\left\{i_{1}, \ldots, i_{k}\right\}$ of $\{1, \ldots, n\}$, we set

$$
\left\{i_{k+1}, \ldots, i_{n}\right\}=\{1, \ldots, n\} \backslash\left\{i_{1}, \ldots, i_{k}\right\} .
$$

Then, we denote by

$$
\mathcal{K}=\left\{K_{i_{1} \cdots i_{k}} \overline{i_{k+1}} \cdots \overline{i_{n}},\left\{i_{1}, \ldots, i_{k}\right\} \subseteq\{1, \ldots, n\}\right\}
$$

where

$$
K_{i_{1} \cdots i_{k} \overline{i_{k+1}} \cdots \bar{i}_{n}}=\left(\bigwedge_{i \in\left\{i_{1}, \ldots, i_{k}\right\}} E_{i} H_{i}\right) \wedge\left(\bigwedge_{i \in\left\{i_{k+1}, \ldots, i_{n}\right\}} \bar{E}_{i} H_{i}\right)=E_{i_{1}} H_{i_{1}} \cdots E_{i_{k}} H_{i_{k}} \bar{E}_{i_{k+1}} H_{i_{k+1}} \cdots \bar{E}_{i_{n}} H_{i_{n}},
$$


the set of $2^{n}$ constituents $C_{h}$ 's contained in $\bigwedge_{i=1}^{n} H_{i}$, that is $\mathcal{K}=\left\{K_{1 \cdots n}, K_{1 \cdots n-1 \bar{n}}, \ldots, K_{\overline{1} \cdots \bar{n}}\right\}$. Of course, there are $3^{n}-2^{n}$ constituents $C_{h}$ 's which logically imply $\bigvee_{i=1}^{n} \bar{H}_{i}$ and hence do not belong to $\mathcal{K}$. For each subset $\left\{i_{1}, \ldots, i_{k}\right\} \subseteq\{1, \ldots, n\}$, we denote by $Q_{i_{1} \ldots i_{k} \overline{k_{k+1}} \ldots \bar{i}_{n}}$ the point associated with the constituent $K_{i_{1} \cdots i_{k} \overline{i_{k+1}} \cdots \overline{i_{n}}} \in \mathcal{K}$. Each point $Q_{i_{1} \cdots i_{k} \overline{i_{k+1}} \ldots \overline{i_{n}}}$ is a $(n+1)$-vector, say $\left(q_{1}, \ldots, q_{n+1}\right)$, where

$$
q_{j}=\left\{\begin{array}{l}
1, \text { if } j \in\left\{i_{1}, \ldots, i_{k}\right\}, \\
0, \text { if } j \in\left\{i_{k+1}, \ldots, i_{n}\right\},
\end{array} \quad q_{n+1}=\left\{\begin{array}{l}
1, \text { if } k=n, \\
0, \text { if } k<n .
\end{array}\right.\right.
$$

Then, from (17), the set of points $\left\{Q_{i_{1} \cdots i_{k} \overline{k_{k+1}} \cdots \bar{i}_{n}},\left\{i_{1}, \ldots, i_{k}\right\} \subseteq\{1, \ldots, n\}\right\}$, which we also denote by $\left\{Q_{1 \cdots n}\right.$, $\left.Q_{1 \cdots n-1 \bar{n}}, \ldots, Q_{\overline{1} \cdots \bar{n}}\right\}$, is $\{(1, \ldots, 1,1),(1, \ldots 1,0,0), \ldots,(0, \ldots, 0)\}$. We denote by $\mathcal{I}^{*}$ the convex hull of these $2^{n}$ points, that is

$$
\mathcal{I}^{*}=\left\{\mathcal{M}: \mathcal{M}=\lambda_{1 \cdots n} Q_{1 \cdots n}+\cdots+\lambda_{\overline{1} \cdots \bar{n}} Q_{\overline{1} \cdots \bar{n}} ; \lambda_{1 \cdots n}+\cdots+\lambda_{\overline{1} \cdots \bar{n}}=1 ; \lambda_{1 \cdots n} \geqslant 0, \ldots, \lambda_{\overline{1} \cdots \bar{n}} \geqslant 0\right\} .
$$

Moreover, we denote by $\left(\Sigma_{n}^{*}\right)$ the following system, with $2^{n}$ unknowns $\lambda_{i_{1} \cdots i_{k}} \overline{i_{k+1}} \ldots \overline{i_{n}}$,

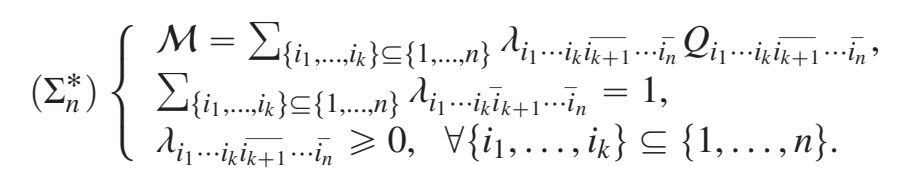

which is solvable if and only if $\mathcal{M} \in \mathcal{I}^{*}$. In more explicit terms the system $\left(\Sigma_{n}^{*}\right)$ becomes

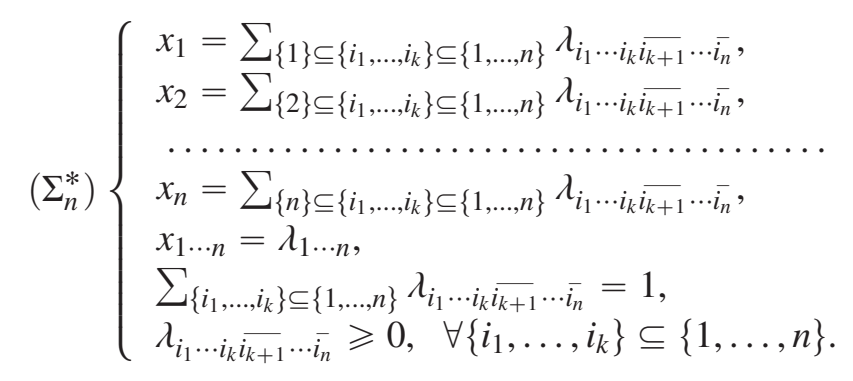

Remark 3. Let $I$ be the convex hull of all the points $Q_{h}$ 's associated with all the constituents $C_{h}$ 's in $H_{1} \vee \cdots \vee H_{n}$. Of course, for each $C_{h} \in \mathcal{K}$, it holds that $C_{h} \subseteq \bigwedge_{i=1}^{n} H_{i} \subseteq \bigvee_{i=1}^{n} H_{i}$. Then, the convex hull $\mathcal{I}^{*}$ is a subset of $\mathcal{I}$.

Theorem 9. Let $E_{1}, \ldots, E_{n}, H_{1}, \ldots, H_{n}$ be logically independent events, with $H_{1} \neq \varnothing, \ldots, H_{n} \neq \varnothing$, $n \geqslant 2$. Moreover, let $\mathcal{M}=\left(x_{1}, \ldots, x_{n}, x_{1 \ldots n}\right)$ be a prevision assessment on $\mathcal{F}=\left\{E_{1}\left|H_{1}, \ldots, E_{n}\right| H_{n}, \mathcal{C}_{1 \ldots n}\right\}$. If $\mathcal{M} \in \mathcal{I}^{*}$, that is $\left(\Sigma_{n}^{*}\right)$ is solvable, then $\mathcal{M}$ is coherent.

Proof. Let be $\mathcal{M} \in \mathcal{I}^{*}$, that is $\left(\Sigma_{n}^{*}\right)$ solvable, with a solution $\Lambda^{*}$. From Remark 3 , as $\mathcal{I}^{*} \subseteq \mathcal{I}$, where $\mathcal{I}$ is the convex hull of all the points $Q_{h}$ 's, $h=1, \ldots, m$, it holds that $\mathcal{M} \in \mathcal{I}$. Then, the system $(\Sigma)$ in (3) is solvable with a solution $\Lambda=\left(\lambda_{h}, h=1, \ldots, m\right)=\left(\Lambda^{*}, \mathbf{0}\right)$, that is $\lambda_{h}=0$ for each $C_{h} \nsubseteq \bigwedge_{i=1}^{n} H_{i}$. Moreover, as $\sum_{h: C_{h} \subseteq H_{i}} \lambda_{h}=\sum_{h: C_{h} \subseteq H_{1} \cdots H_{n}} \lambda_{h}=1, i=1, \ldots, n$, it holds that $I_{0}=\varnothing$. Thus, as $(\Sigma)$ is solvable and $I_{0}$ is empty, by Theorem 5] the assessment $\mathcal{M}$ is coherent.

We recall that a t-norm $T$, introduced as a binary operator, can be extended as an $n$-ary operator. For any integer $n \geqslant 2$ the extension of $T$ is defined as ([41]])

$$
T\left(x_{1}, \ldots, x_{n}\right)= \begin{cases}T\left(T\left(x_{1}, \ldots, x_{n-1}\right), x_{n}\right), & \text { if } n>2 \\ T\left(x_{1}, x_{2}\right), & \text { if } n=2\end{cases}
$$


The Fréchet-Hoeffding bounds are

$$
T_{L}\left(x_{1}, \ldots, x_{n}\right)=\max \left\{\sum_{i=1}^{n} x_{i}-n+1,0\right\}, \quad T_{M}\left(x_{1}, \ldots, x_{n}\right)=\min \left\{x_{1}, \ldots, x_{n}\right\} .
$$

In the next result (Theorem 10) we show that, when propagating the probability assessment $\mathcal{P}=$ $\left(x_{1}, \ldots, x_{n}\right)$, defined on the family of $n$ conditional events $\left\{E_{1}\left|H_{1}, \ldots, E_{n}\right| H_{n}\right\}$, to their conjunction $\mathcal{C}_{1 \ldots n}$, under logical independence the prevision assessment $\mathbb{P}\left(\mathcal{C}_{1 \ldots n}\right)=\mu$ is a coherent extension of $\mathcal{P}$ if and only if $\mu \in\left[\mu^{\prime}\left(x_{1}, \ldots, x_{n}\right), \mu^{\prime \prime}\left(x_{1}, \ldots, x_{n}\right)\right]$, where

$$
\mu^{\prime}\left(x_{1}, \ldots, x_{n}\right)=T_{L}\left(x_{1}, \ldots, x_{n}\right), \mu^{\prime \prime}\left(x_{1}, \ldots, x_{n}\right)=T_{M}\left(x_{1}, \ldots, x_{n}\right) .
$$

For the convenience of the reader we sketch the proof.

1. We first observe that in general it holds that

$$
T_{L}\left(x_{1}, \ldots, x_{n}\right) \leqslant \mu^{\prime}\left(x_{1}, \ldots, x_{n}\right) \leqslant \mu^{\prime \prime}\left(x_{1}, \ldots, x_{n}\right) \leqslant T_{M}\left(x_{1}, \ldots, x_{n}\right) .
$$

2. We show that $\mu^{\prime}\left(x_{1}, \ldots, x_{n}\right)=T_{L}\left(x_{1}, \ldots, x_{n}\right)$, by verifying the coherence of the assessment $\left(x_{1}, \ldots, x_{n}, T_{L}\left(x_{1}, \ldots, x_{n}\right)\right)$ on $\left\{E_{1}\left|H_{1}, \ldots, E_{n}\right| H_{n}, \mathcal{C}_{1 \ldots n}\right\}$. Based on Theorem 9 , we verify the coherence of $\left(x_{1}, \ldots, x_{n}, T_{L}\left(x_{1}, \ldots, x_{n}\right)\right)$ by showing that the associated system $\left(\Sigma_{n}^{*}\right)$ is solvable, for each $n$. We proceed by induction. We assume $\left(\Sigma_{n}^{*}\right)$ solvable and then we verify the solvability of $\left(\Sigma_{n+1}^{*}\right)$, by separately examining two cases: $(i) T_{L}\left(x_{1}, \ldots, x_{n}\right)=0$, (ii) $T_{L}\left(x_{1}, \ldots, x_{n}\right)>0$. In the case $(i)$ for the assessment $P\left(E_{n+1} \mid H_{n+1}\right)=x_{n+1}$ we distinguish three sub-cases: $x_{n+1}=0, x_{n+1}=1,0<x_{n+1}<1$. In the case $(i i)$ we give an explicit solution of $\left(\Sigma_{n}^{*}\right)$ and a related solution for $\left(\Sigma_{n+1}^{*}\right)$, by distinguishing two sub-cases which concern $x_{n+1}$ : (ii.a) $0 \leqslant x_{n+1} \leqslant n-\sum_{i=1}^{n}$ (with three further sub-cases); (ii.b) $n-\sum_{i=1}^{n}<x_{n+1} \leqslant 1$.

3. We show that $\mu^{\prime \prime}\left(x_{1}, \ldots, x_{n}\right)=T_{M}\left(x_{1}, \ldots, x_{n}\right)$. We verify the coherence of the assessment $\left(x_{1}, \ldots, x_{n}, T_{M}\left(x_{1}, \ldots, x_{n}\right)\right)$ on $\left\{E_{1}\left|H_{1}, \ldots, E_{n}\right| H_{n}, \mathcal{C}_{1 \ldots n}\right\}$, by providing an explicit solution of $\left(\Sigma_{n}^{*}\right)$ and by applying Theorem 9

Theorem 10. Let $E_{1}, \ldots, E_{n}, H_{1}, \ldots, H_{n}$ be logically independents events, with $H_{1} \neq \varnothing, \ldots, H_{n} \neq \varnothing$, $n \geqslant 2$. The set $\Pi$ of all prevision coherent assessments $\mathcal{M}=\left(x_{1}, \ldots, x_{n}, x_{1 \ldots n}\right)$ on the family $\mathcal{F}=$ $\left\{E_{1}\left|H_{1}, \ldots, E_{n}\right| H_{n}, \mathcal{C}_{1 \ldots n}\right\}$ is

$$
\Pi=\left\{\left(x_{1}, \ldots, x_{n}, x_{1 \ldots n}\right):\left(x_{1}, \ldots, x_{n}\right) \in[0,1]^{n}, x_{1 \ldots n} \in\left[T_{L}\left(x_{1}, \ldots, x_{n}\right), T_{M}\left(x_{1}, \ldots, x_{n}\right)\right]\right\} .
$$

Proof. Given any integer $n \geqslant 2$, by logical independence of $E_{1}, \ldots, E_{n}, H_{1}, \ldots, H_{n}$ each point $\left(x_{1}, \ldots, x_{n}\right) \in$ $[0,1]^{n}$ is a coherent assessment on $\left\{E_{1}\left|H_{1}, \ldots, E_{n}\right| H_{n}\right\}$ ([25, Proposition 11]). Moreover, by Theorem 6 , for each $\left(x_{1}, \ldots, x_{n}\right) \in[0,1]^{n}$ there exist two values $\mu^{\prime}\left(x_{1}, \ldots, x_{n}\right)$ and $\mu^{\prime \prime}\left(x_{1}, \ldots, x_{n}\right)$ such that $x_{1 \ldots n}$ is a coherent extension of $\left(x_{1}, \ldots, x_{n}\right)$ if and only if $x_{1 \ldots n} \in\left[\mu^{\prime}\left(x_{1}, \ldots, x_{n}\right), \mu^{\prime \prime}\left(x_{1}, \ldots, x_{n}\right)\right]$. Then,

$$
\Pi=\left\{\left(x_{1}, \ldots, x_{n}, x_{1 \ldots n}\right):\left(x_{1}, \ldots, x_{n}\right) \in[0,1]^{n}, x_{1 \ldots n} \in\left[\mu^{\prime}\left(x_{1}, \ldots, x_{n}\right), \mu^{\prime \prime}\left(x_{1}, \ldots, x_{n}\right)\right]\right\} .
$$

By Theorem 8 , coherence requires that $x_{1 \ldots n} \in\left[T_{L}\left(x_{1}, \ldots, x_{n}\right), T_{M}\left(x_{1}, \ldots, x_{n}\right)\right]$ and hence

$$
T_{L}\left(x_{1}, \ldots, x_{n}\right) \leqslant \mu^{\prime}\left(x_{1}, \ldots, x_{n}\right) \leqslant \mu^{\prime \prime}\left(x_{1}, \ldots, x_{n}\right) \leqslant T_{M}\left(x_{1}, \ldots, x_{n}\right) .
$$

Thus, $\Pi \subseteq\left\{\left(x_{1}, \ldots, x_{n}, x_{1 \ldots n}\right):\left(x_{1}, \ldots, x_{n}\right) \in[0,1]^{n}, x_{1 \ldots n} \in\left[T_{L}\left(x_{1}, \ldots, x_{n}\right), T_{M}\left(x_{1}, \ldots, x_{n}\right)\right]\right\} . \quad$ In order to complete the proof it is enough to show that the two assessments $\left(x_{1}, \ldots, x_{n}, T_{L}\left(x_{1}, \ldots, x_{n}\right)\right)$ and $\left(x_{1}, \ldots, x_{n}, T_{M}\left(x_{1}, \ldots, x_{n}\right)\right)$ are coherent, for every $\left(x_{1}, \ldots, x_{n}\right) \in[0,1]^{n}$, that is $\mu^{\prime}\left(x_{1}, \ldots, x_{n}\right)=$ $T_{L}\left(x_{1}, \ldots, x_{n}\right)$ and $\mu^{\prime \prime}\left(x_{1}, \ldots, x_{n}\right)=T_{M}\left(x_{1}, \ldots, x_{n}\right)$, which amounts to the sharpness of the FréchetHoeffding bounds. 
Coherence of $\left(x_{1}, \ldots, x_{n}, T_{L}\left(x_{1}, \ldots, x_{n}\right)\right)$. We will proceed by induction on the solvability of the system $\left(\Sigma_{n}^{*}\right)$ associated to the assessment $\left(x_{1}, \ldots, x_{n}, T_{L}\left(x_{1}, \ldots, x_{n}\right)\right)$.

$(n=2)$. In this case, by Remark 2 , the assessment $\mathcal{M}=\left(x_{1}, x_{2}, T_{L}\left(x_{1}, x_{2}\right)\right)$ is coherent because $\mathcal{M}$ belongs to the set of coherent prevision assessments, given by the tetrahedron with vertices the points $(1,1,1),(1,0,0),(0,1,0),(0,0,0)$. Moreover, by recalling (16), for $n=2$ the constituents which logically imply $H_{1} H_{2}$ are

$$
K_{11}=E_{1} H_{1} E_{2} H_{2}, \quad K_{1 \overline{2}}=E_{1} H_{1} \bar{E}_{2} H_{2}, \quad K_{\overline{1} 2}=\bar{E}_{1} H_{1} E_{2} H_{2}, \quad K_{\overline{1} \overline{2}}=\bar{E}_{1} H_{1} \bar{E}_{2} H_{2} .
$$

The associated points are

$$
Q_{11}=(1,1,1), \quad Q_{1 \overline{2}}=(1,0,0), \quad Q_{\overline{1} 2}=(0,1,0), \quad Q_{\overline{1} \overline{2}}=(0,0,0) ;
$$

Thus, the convex hull $\mathcal{I}^{*}$ of $Q_{12}, Q_{1 \overline{2}}, Q_{\overline{1} 2}, Q_{\overline{12}}$ coincides with the tetrahedron and hence $\mathcal{M} \in \mathcal{I}^{*}$, that is $\left(\Sigma_{2}^{*}\right)$ is solvable. Indeed, $\left(\Sigma_{2}^{*}\right)$ is the following system

$$
\left(\Sigma_{2}^{*}\right)\left\{\begin{array}{l}
x_{1}=\lambda_{12}+\lambda_{1 \overline{1}} \\
x_{2}=\lambda_{12}+\lambda_{\overline{1} 2} \\
T_{L}\left(x_{1}, x_{2}\right)=\lambda_{12} \\
\lambda_{12}+\lambda_{1 \overline{2}}+\lambda_{\overline{1} 2}+\lambda_{\overline{1} \overline{2}}=1 \\
\lambda_{i_{1} i_{2}} \geqslant 0, \quad \forall\left(i_{1}, i_{2}\right) \in\{1, \overline{1}\} \times\{2, \overline{2}\}
\end{array}\right.
$$

with a solution

$$
\Lambda_{2}=\left(\lambda_{12}, \lambda_{\overline{1} 2}, \lambda_{1 \overline{2}}, \lambda_{\overline{1} \overline{2}}\right)=\left(T_{L}\left(x_{1}, x_{2}\right), x_{2}-T_{L}\left(x_{1}, x_{2}\right), x_{1}-T_{L}\left(x_{1}, x_{2}\right), 1-x_{1}-x_{2}+T_{L}\left(x_{1}, x_{2}\right)\right) .
$$

In particular

$$
\Lambda_{2}=\left\{\begin{array}{l}
\left(0, x_{2}, x_{1}, 1-x_{1}-x_{2}\right), \text { if } x_{1}+x_{2}-1 \leqslant 0, \\
\left(x_{1}+x_{2}-1,1-x_{1}, 1-x_{2}, 0\right), \text { if } x_{1}+x_{2}-1>0 .
\end{array}\right.
$$

We now assume the system $\left(\Sigma_{n}^{*}\right)$ associated with the assessment $\left(x_{1}, \ldots, x_{n}, T_{L}\left(x_{1}, \ldots, x_{n}\right)\right)$ is solvable and we show that the system $\left(\Sigma_{n+1}^{*}\right)$ associated with the assessment $\left(x_{1}, \ldots, x_{n+1}, T_{L}\left(x_{1}, \ldots, x_{n+1}\right)\right)$ is solvable too. Then, $\left(\Sigma_{n}^{*}\right)$ is solvable for every $n \geqslant 2$ and by Theorem 9 it follows the coherence of the assessment $\left(x_{1}, \ldots, x_{n}, T_{L}\left(x_{1}, \ldots, x_{n}\right)\right)$, for every $n$.

Let the vector $\Lambda_{n}=\left(\lambda_{i_{1} \cdots i_{k}} \overline{i_{k+1} \cdots \bar{i}_{n}}:\left\{i_{1}, \ldots, i_{k}\right\} \subseteq\{1, \ldots, n\}\right)$ be a solution of $\left(\Sigma_{n}^{*}\right)$. Then, by (20), $\lambda_{1 \cdots n}=x_{1 \cdots n}=T_{L}\left(x_{1}, \ldots, x_{n}\right)$. When necessary we assume that the components of $\Lambda_{n}$ are suitably ordered. Given a further conditional event $E_{n+1} \mid H_{n+1}$, with $P\left(E_{n+1} \mid H_{n+1}\right)=x_{n+1}$, the system $\left(\Sigma_{n+1}^{*}\right)$ associated with the assessment $\left(x_{1}, \ldots, x_{n+1}, T_{L}\left(x_{1}, \ldots, x_{n+1}\right)\right)$ on $\left\{E_{1}\left|H_{1}, \ldots, E_{n+1}\right| H_{n+1}, \mathrm{C}_{1 \cdots n+1}\right\}$ is

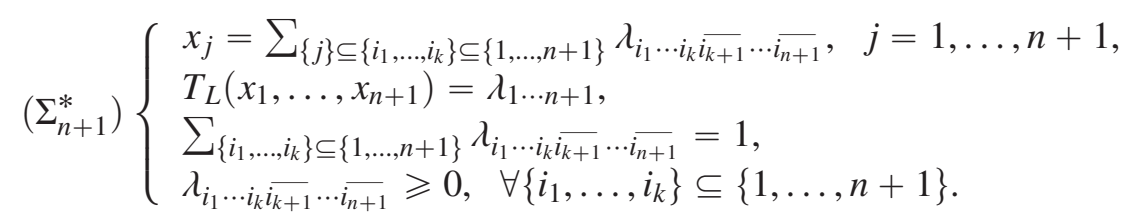

Based on $\Lambda_{n}$ we will find a solution $\Lambda_{n+1}$ of $\left(\Sigma_{n+1}^{*}\right)$ such that

$$
\lambda_{i_{1} \cdots i_{k} \overline{i_{k+1}} \cdots \overline{i_{n}}}=\lambda_{i_{1} \cdots i_{k} \overline{i_{k+1}} \cdots \overline{i_{n}} n+1}+\lambda_{i_{1} \cdots i_{k} \overline{i_{k+1}} \cdots \overline{i_{n}} \overline{n+1}}, \quad\left\{i_{1}, \ldots, i_{k}\right\} \subseteq\{1, \ldots, n\} .
$$


Then, the system $\left(\Sigma_{n+1}^{*}\right)$ becomes

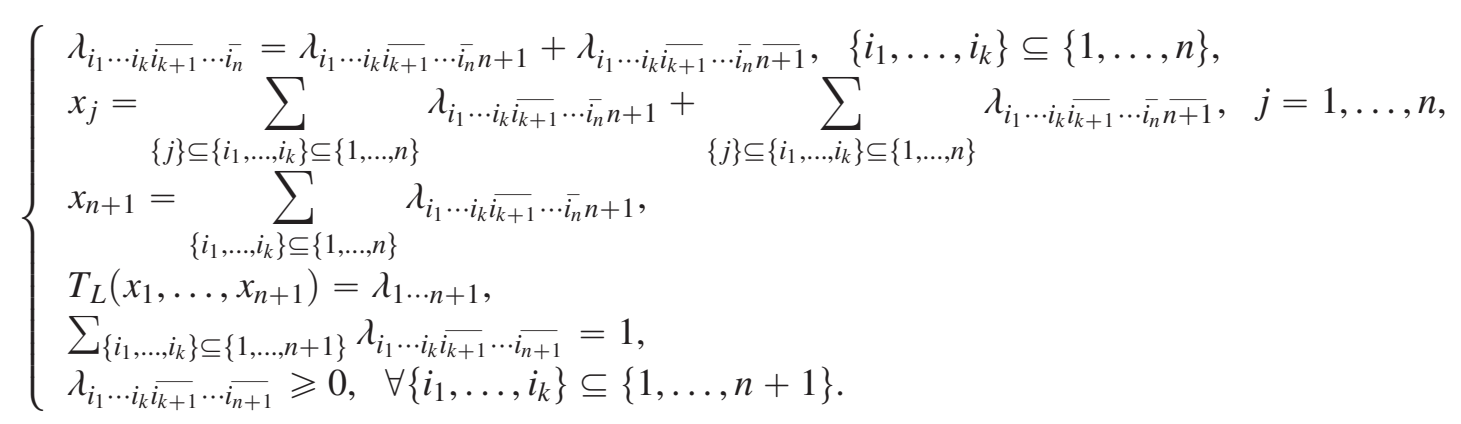

We distinguish two cases: $(i) T_{L}\left(x_{1}, \ldots, x_{n}\right)=0$, that is $x_{1}+\cdots+x_{n}-n+1 \leqslant 0$; (ii) $T_{L}\left(x_{1}, \ldots, x_{n}\right)>0$, that is $x_{1}+\cdots+x_{n}-n+1>0$.

Case $(i)$. As $T_{L}\left(x_{1}, \ldots, x_{n}\right)=0$, it holds that $T_{L}\left(x_{1}, \ldots, x_{n+1}\right)=T_{L}\left(T_{L}\left(x_{1}, \ldots, x_{n}\right), x_{n+1}\right)=$ $T_{L}\left(0, x_{n+1}\right)=0$. Moreover, for the component $\lambda_{1 \cdots n}$ of the vector $\Lambda_{n}$ it holds that $\lambda_{1 \cdots n}=0$; hence, in (25), $\lambda_{1 \cdots n n+1}=0$ and $\lambda_{1 \cdots n n+1}=0$, which satisfies the equation $T_{L}\left(x_{1}, \ldots, x_{n+1}\right)=\lambda_{1 \cdots n+1}$. We first examine the particular cases where $x_{n+1}=0$, or $x_{n+1}=1$; then, we consider the case $0<x_{n+1}<1$.

If $x_{n+1}=0$, the system (25) becomes

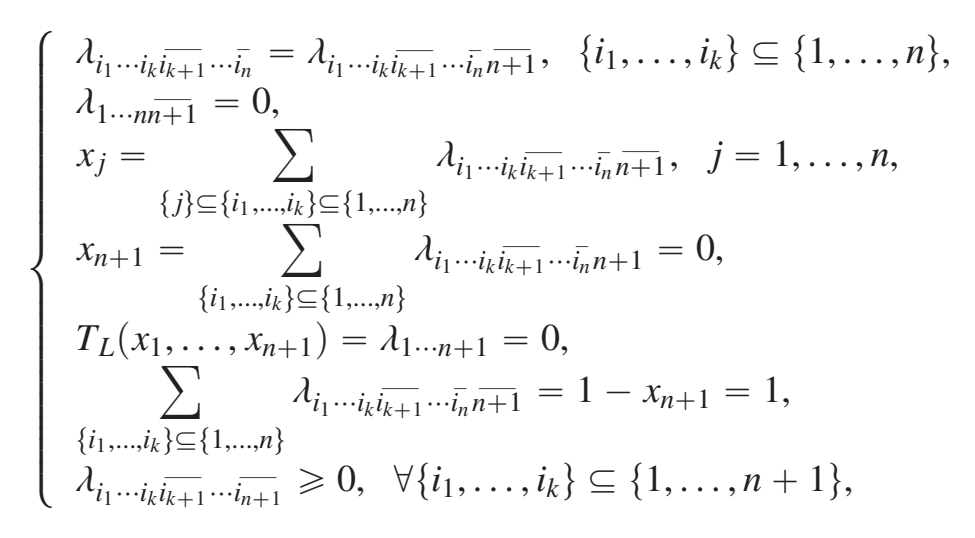

with a solution $\Lambda_{n+1,0}$ given by

$$
\Lambda_{n+1,0}=\left(\lambda_{i_{1} \cdots i_{k} \overline{i_{k+1}} \cdots \overline{i_{n}} n+1}, \lambda_{i_{1} \cdots i_{k}} \overline{i_{k+1}} \cdots \overline{i_{n}} \overline{n+1},\left\{i_{1}, \ldots, i_{k}\right\} \subseteq\{1, \ldots, n\}\right),
$$

where $\lambda_{i_{1} \cdots i_{k} \overline{i_{k+1}} \cdots \overline{i_{n}} n+1}=0$ and $\lambda_{i_{1} \cdots i_{k} \overline{i_{k+1}} \cdots \overline{i_{n}} \overline{n+1}}=\lambda_{i_{1} \cdots i_{k} \overline{i_{k+1}} \ldots \overline{i_{n}}},\left\{i_{1}, \ldots, i_{k}\right\} \subseteq\{1, \ldots, n\}$, with in particular $\lambda_{1 \cdots n n+1}=\lambda_{1 \cdots n}=0$. Thus $\left(\Sigma_{n+1}^{*}\right)$ is solvable when $T_{L}\left(x_{1}, \ldots, x_{n}\right)=0$ and $x_{n+1}=0$. We also observe that $\Lambda_{n+1,0}$ has the following structure

$$
\left(\lambda_{1 \cdots n+1}, \ldots, \lambda_{\overline{1} \cdots \bar{n} n+1}, \lambda_{1 \cdots n \overline{n+1}}, \ldots, \lambda_{\overline{1} \cdots \bar{n} \overline{n+1}}\right)=\left(0, \ldots, 0, \lambda_{1 \cdots n}, \ldots, \lambda_{\overline{1} \cdots \bar{n}}\right)=\left(\mathbf{0}_{n}, \Lambda_{n}\right),
$$

where $\mathbf{0}_{n}$ is the subvector $(0, \ldots, 0)$ of length $2^{n}$ and $\Lambda_{n}$ is a solution of $\left(\Sigma_{n}^{*}\right)$. 
If $x_{n+1}=1$, the system (25) becomes

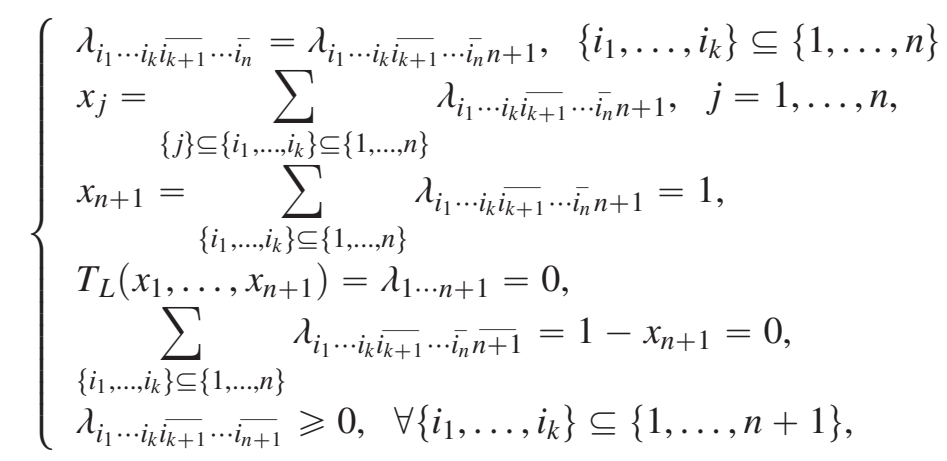

with a solution $\Lambda_{n+1,1}$ given by

$$
\Lambda_{n+1,1}=\left(\lambda_{i_{1} \cdots i_{k} \overline{i_{k+1}} \cdots \overline{i_{n}} n+1}, \lambda_{i_{1} \cdots i_{k}} \overline{i_{k+1}} \cdots \overline{i_{n}} \overline{n+1},\left\{i_{1}, \ldots, i_{k}\right\} \subseteq\{1, \ldots, n\}\right),
$$

where $\lambda_{i_{1} \cdots i_{k} \overline{i_{k+1}} \cdots \overline{i_{n}} n+1}=\lambda_{i_{1} \cdots i_{k}} \overline{i_{k+1}} \cdots \overline{i_{n}}$ and $\lambda_{i_{1} \cdots i_{k} \overline{i_{k+1}} \cdots \overline{i_{n}} \overline{n+1}}=0,\left\{i_{1}, \ldots, i_{k}\right\} \subseteq\{1, \ldots, n\}$, with in particular $\lambda_{1 \cdots n \overline{n+1}}=\lambda_{1 \cdots n}=0$. Thus $\left(\Sigma_{n+1}^{*}\right)$ is solvable when $T_{L}\left(x_{1}, \ldots, x_{n}\right)=0$ and $x_{n+1}=1$. We also observe that $\Lambda_{n+1,1}$ has the following structure

$$
\begin{aligned}
& \Lambda_{n+1,1}=\left(\lambda_{1 \cdots n+1}, \ldots, \lambda_{\overline{1} \ldots \bar{n} n+1}, \lambda_{1 \cdots n \bar{n}}, \ldots, \lambda_{\overline{1} \cdots \bar{n} \overline{n+1}}\right)= \\
& =\left(\lambda_{1 \cdots n}, \ldots, \lambda_{\overline{1} \ldots \bar{n}}, 0, \ldots, 0\right)=\left(\Lambda_{n}, \mathbf{0}_{n}\right) .
\end{aligned}
$$

If $0<x_{n+1}<1$, by observing that $\left(x_{1}, \ldots, x_{n+1}, T_{L}\left(x_{1}, \ldots, x_{n+1}\right)\right)=\left(x_{1}, \ldots, x_{n+1}, 0\right)$, as

$$
\left(x_{1}, \ldots, x_{n+1}, 0\right)=\left(1-x_{n+1}\right) \cdot\left(x_{1}, \ldots, x_{n}, 0,0\right)+x_{n+1} \cdot\left(x_{1}, \ldots, x_{n}, 1,0\right),
$$

the vector $\Lambda_{n+1}=\left(1-x_{n+1}\right) \Lambda_{n+1,0}+x_{n+1} \Lambda_{n+1,1}$ is a solution of system (25); thus $\left(\Sigma_{n+1}^{*}\right)$ is solvable when $T_{L}\left(x_{1}, \ldots, x_{n}\right)=0$ and $0<x_{n+1}<1$.

Therefore, by exploiting the solution $\Lambda_{n}$ of $\left(\Sigma_{n}^{*}\right)$, when $T_{L}\left(x_{1}, \ldots, x_{n}\right)=0$ the system $\left(\Sigma_{n+1}^{*}\right)$ is solvable for every $x_{n+1} \in[0,1]$, with a solution given by

$$
\begin{aligned}
& \Lambda_{n+1}=\left(\lambda_{1 \cdots n+1}, \ldots, \lambda_{\overline{1} \ldots \bar{n} n+1}, \lambda_{1 \ldots n \overline{n+1}}, \ldots, \lambda_{\overline{1} \cdots \bar{n} \overline{n+1}}\right)=\left(1-x_{n+1}\right) \Lambda_{n+1,0}+x_{n+1} \Lambda_{n+1,1}= \\
& =\left(1-x_{n+1}\right)\left(\mathbf{0}_{n}, \Lambda_{n}\right)+x_{n+1}\left(\Lambda_{n}, \mathbf{0}_{n}\right)=\left(x_{n+1} \Lambda_{n},\left(1-x_{n+1}\right) \Lambda_{n}\right)= \\
& =\left(x_{n+1} \lambda_{1 \cdots n}, \ldots, x_{n+1} \lambda_{\overline{1} \cdots \bar{n}},\left(1-x_{n+1}\right) \lambda_{1 \cdots n}, \ldots,\left(1-x_{n+1}\right) \lambda_{1} \cdots \bar{n}\right) .
\end{aligned}
$$

Case $(i i)$. In this case $T_{L}\left(x_{1}, \ldots, x_{n}\right)=x_{1}+\ldots+x_{n}-(n-1)>0$ and by the inductive hypothesis the system $\left(\Sigma_{n}^{*}\right)$ is solvable. Actually, an explicit solution of $\left(\Sigma_{n}^{*}\right)$ is the nonnegative vector $\Lambda_{n}=\left(\lambda_{i_{1} \cdots i_{k}} \overline{i_{k+1}} \cdots \overline{i_{n}},\left\{i_{1}, \ldots, i_{k}\right\} \subseteq\{1, \ldots, n\}\right)$ given by

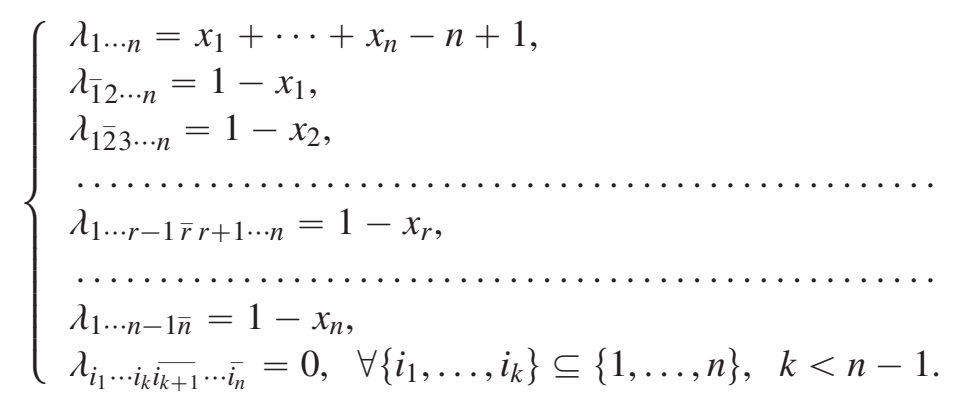


Indeed, $\Lambda_{n}$ is a solution of $\left(\Sigma_{n}^{*}\right)$ as shown below

$$
\left\{\begin{array}{l}
x_{j}=\lambda_{1 \cdots n}+\sum_{k=1}^{n} \lambda_{1 \cdots k-1 \bar{k} k+1 \cdots n}-\lambda_{1 \cdots j-1} j j+1 \cdots n \\
T_{L}\left(x_{1}, \ldots, x_{n}\right)=\lambda_{1 \cdots n}, \\
\lambda_{i_{1} \cdots i_{k} \overline{i_{k+1}} \cdots \bar{i}_{n}}=0, \quad \forall\left\{i_{1}, \ldots, i_{k}\right\} \subseteq\{1, \ldots, n\}, k<n-1, \\
\sum_{\left\{i_{1}, \ldots, i_{k}\right\} \subseteq\{1, \ldots, n\}} \lambda_{i_{1} \cdots i_{k}} \overline{i_{k+1}} \cdots \overline{i_{n}}=\lambda_{1 \cdots n}+\sum_{k=1}^{n} \lambda_{1 \cdots k-1 \bar{k} k+1 \cdots n}=1 .
\end{array}\right.
$$

Based on (31) the system (25) becomes

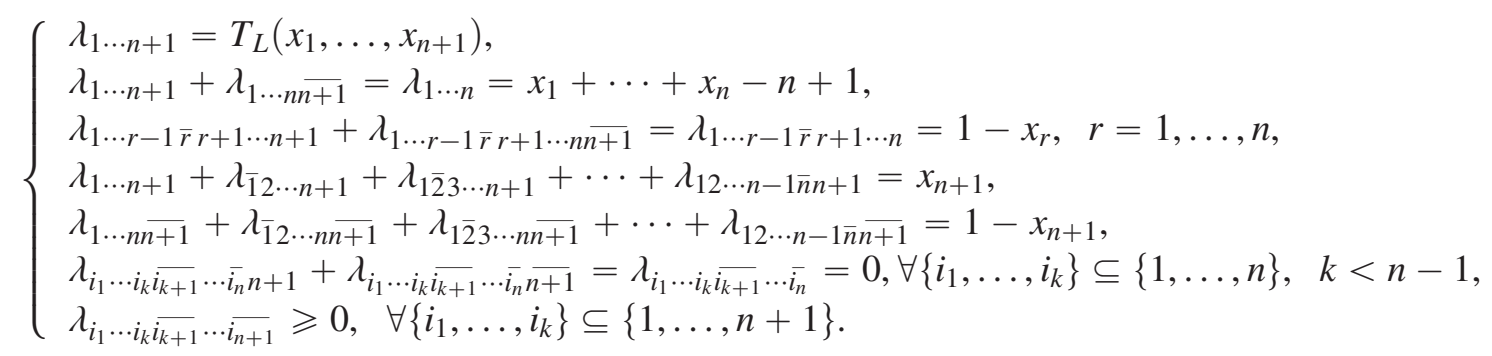

Based on the solution of $\left(\Sigma_{n}^{*}\right)$ given in (31) we find a solution of (33), which of course is also a solution of $\left(\Sigma_{n+1}^{*}\right)$. We observe that, as $T_{L}\left(x_{1}, \ldots, x_{n}\right)>0$, it holds that $n-\sum_{i=1}^{n} x_{i}=1-T_{L}\left(x_{1}, \ldots, x_{n}\right)<1$; then, we distinguish two sub-cases which concern $x_{n+1}$ :

(ii.a) $0 \leqslant x_{n+1} \leqslant n-\sum_{i=1}^{n} x_{i}<1$; (ii.b) $n-\sum_{i=1}^{n} x_{i}<x_{n+1} \leqslant 1$.

Sub-case (ii.a). In this case $T_{L}\left(x_{1}, \ldots, x_{n+1}\right)=0$. We analyze separately three cases:

(ii.a.1) $x_{n+1}=0$; (ii.a.2) $x_{n+1}=n-x_{1}-\cdots-x_{n}$; (ii.a.3) $0<x_{n+1}<n-x_{1}-\cdots-x_{n}$.

In case (ii.a.1), as $x_{n+1}=0$, the system (33) becomes

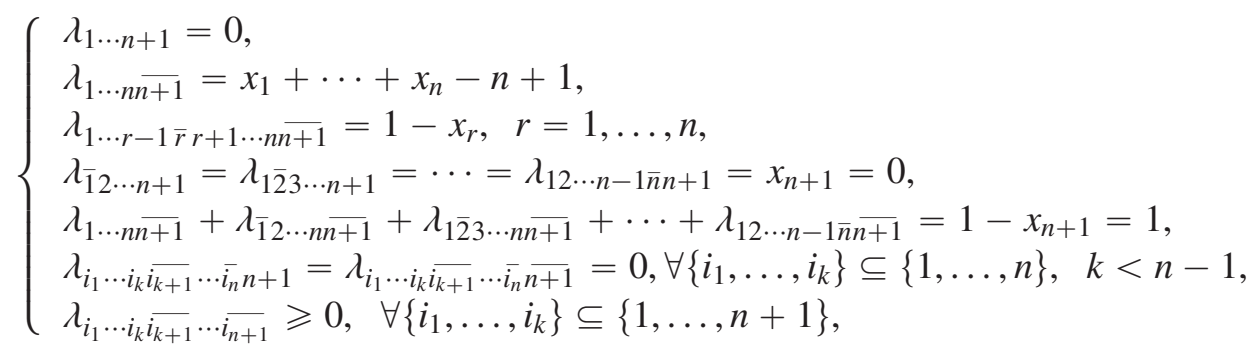

with a solution $\Lambda_{n+1,0}$ given by

$$
\Lambda_{n+1,0}=\left(\lambda_{1 \cdots n+1}, \ldots, \lambda_{\overline{1} \cdots \bar{n} n+1}, \lambda_{1 \cdots n \overline{n+1}}, \ldots, \lambda_{\overline{1} \ldots \bar{n} \overline{n+1}}\right),
$$

where

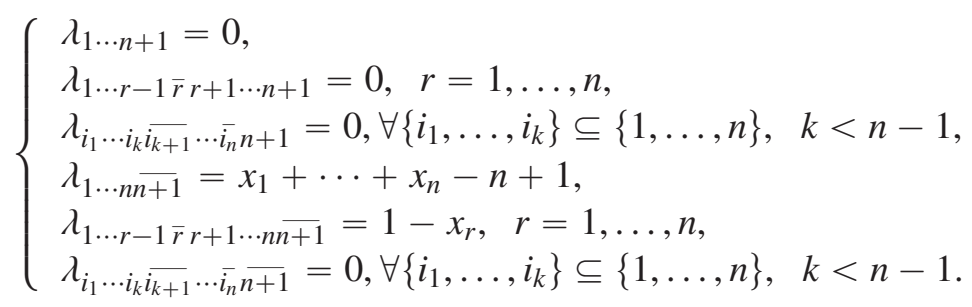

Thus, $\left(\Sigma_{n+1}^{*}\right)$ is solvable when $T_{L}\left(x_{1}, \ldots, x_{n}\right)>0$ and $x_{n+1}=0$. We observe that, from (33) and (35), it holds that

$$
\Lambda_{n+1,0}=\left(0, \ldots, 0, \lambda_{1 \cdots n}, \ldots, \lambda_{\overline{1} \ldots \bar{n}}\right)=\left(\mathbf{0}_{n}, \Lambda_{n}\right) .
$$


In case (ii.a.2), we preliminarily observe that, if $n-\left(x_{1}+\cdots+x_{n}\right)=\sum_{r=1}^{n}\left(1-x_{r}\right)=0$, that is $x_{r}=1$, $r=1, \ldots, n$ and hence $T_{L}\left(x_{1}, \ldots, x_{n}\right)=1$, then $x_{n+1}=n-\left(x_{1}+\cdots+x_{n}\right)=0$, which is the case (ii.a.1) considered before. In this case a solution $\Lambda_{n+1}$ of $\left(\Sigma_{n+1}^{*}\right)$ is the vector $\Lambda_{n+1,0}$ given in (35), which becomes

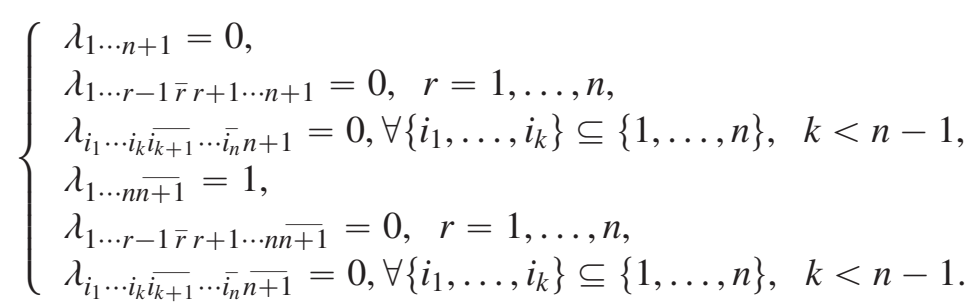

If $0<x_{n+1}=n-\left(x_{1}+\cdots+x_{n}\right)<1$, then the system (33) becomes

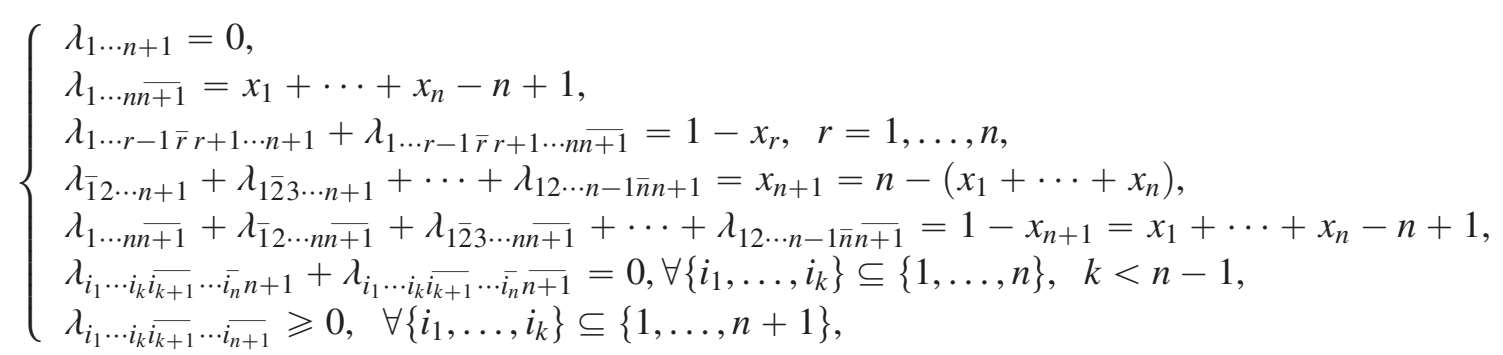

which, by setting $s=n-\left(x_{1}+\cdots+x_{n}\right)$, is solvable with a solution given by

$$
\Lambda_{n+1, s}=\left(\lambda_{1 \cdots n+1}, \ldots, \lambda_{\overline{1} \ldots \bar{n} n+1}, \lambda_{1 \cdots n \overline{n+1}}, \ldots, \lambda_{\overline{1} \ldots \bar{n} \overline{n+1}}\right),
$$

where

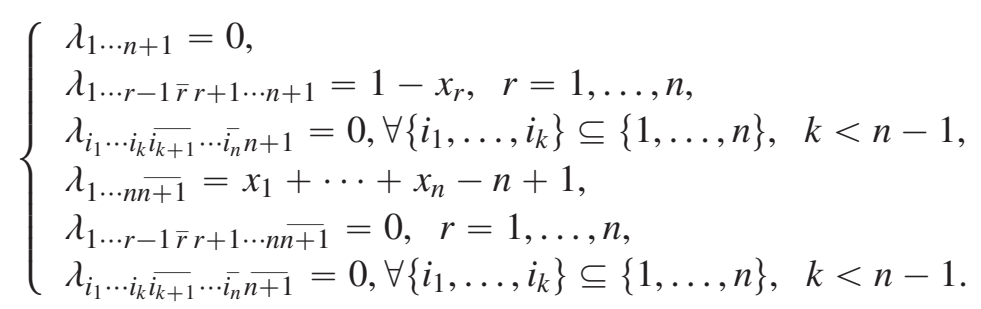

Thus, $\left(\Sigma_{n+1}^{*}\right)$ is solvable when $T_{L}\left(x_{1}, \ldots, x_{n}\right)>0$ and $x_{n+1}=n-\left(x_{1}+\cdots+x_{n}\right)$.

In case (ii.a.3), as the vector $\left(x_{1}, \ldots, x_{n+1}, 0\right)$ coincides with the linear convex combination

$$
\left(1-\frac{x_{n+1}}{n-\left(x_{1}+\cdots+x_{n}\right)}\right) \cdot\left(x_{1}, \ldots, x_{n}, 0,0\right)+\frac{x_{n+1}}{n-\left(x_{1}+\cdots+x_{n}\right)} \cdot\left(x_{1}, \ldots, x_{n}, n-\left(x_{1}+\cdots+x_{n}\right), 0\right),
$$

the vector

$$
\Lambda_{n+1}=\left(1-\frac{x_{n+1}}{n-\left(x_{1}+\cdots+x_{n}\right)}\right) \Lambda_{n+1,0}+\frac{x_{n+1}}{n-\left(x_{1}+\cdots+x_{n}\right)} \Lambda_{n+1, s}
$$

is a solution of system (25); thus $\left(\Sigma_{n+1}^{*}\right)$ is solvable when $T_{L}\left(x_{1}, \ldots, x_{n}\right)>0$ and $0<x_{n+1}<n-\left(x_{1}+\right.$ $\left.\cdots+x_{n}\right)$. We observe that, from (35) and (39), in explicit terms the components of the solution $\Lambda_{n+1}$ in (40) are

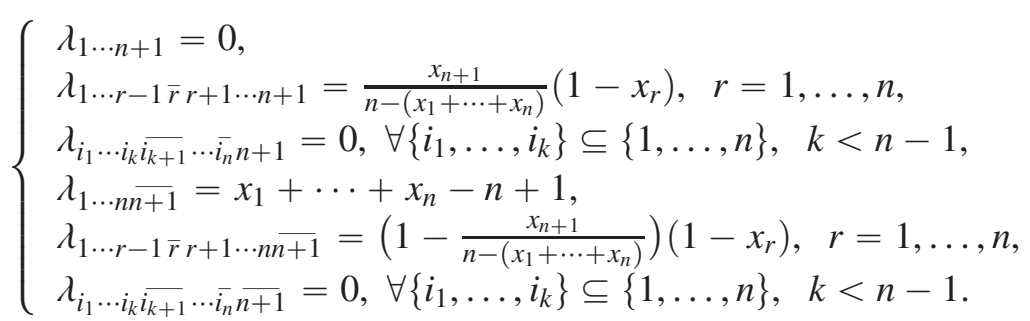


Therefore, by exploiting the solution $\Lambda_{n}$ of $\left(\Sigma_{n}^{*}\right)$, when $T_{L}\left(x_{1}, \ldots, x_{n}\right)>0$ the system $\left(\Sigma_{n+1}^{*}\right)$ is solvable for every $x_{n+1} \in\left[0, n-\left(x_{1}+\cdots+x_{n}\right)\right]$, with a solution given by the vector $\Lambda_{n+1}$ in (40) when $n-\left(x_{1}+\right.$ $\left.\cdots+x_{n}\right)>0$, that is when $T_{L}\left(x_{1}, \ldots, x_{n}\right)<1$, or by the vector given in (37) when $n-\left(x_{1}+\cdots+x_{n}\right)=0$, that is when $T_{L}\left(x_{1}, \ldots, x_{n}\right)=1$.

Sub-case (ii.b). We recall that $n-\left(x_{1}+\cdots+x_{n}\right)=1-T_{L}\left(x_{1}, \ldots, x_{n}\right)<1$ as $T_{L}\left(x_{1}, \ldots, x_{n}\right)>0$. Moreover, $T_{L}\left(x_{1}, \ldots, x_{n+1}\right)=x_{1}+\cdots+x_{n+1}-n>0$, as $n-x_{1}-\cdots-x_{n}<x_{n+1} \leqslant 1$. Similarly to the solution $\Lambda_{n}$ of $\left(\Sigma_{n}^{*}\right)$ given in (31), the system $\left(\Sigma_{n+1}^{*}\right)$ has a solution $\Lambda_{n+1}$ given below.

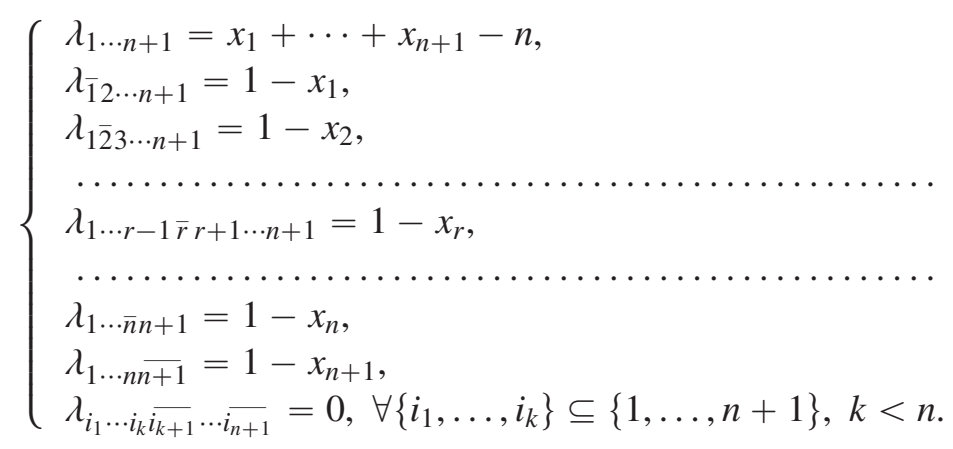

Therefore, based on the solvability of $\left(\Sigma_{n}^{*}\right)$, we showed that in both cases $(i)$ and $(i i)$ system $\left(\Sigma_{n+1}^{*}\right)$ is solvable. In conclusion, $\left(\Sigma_{n}^{*}\right)$ is solvable for every $n \geqslant 2$ and by Theorem 9 the assessment $\left(x_{1}, \ldots, x_{n}, T_{L}\left(x_{1}, \ldots, x_{n}\right)\right)$ on the family $\mathcal{F}=\left\{E_{1}\left|H_{1}, \ldots, E_{n}\right| H_{n}, \mathcal{C}_{1 \ldots n}\right\}$ is coherent for every $n$. Then, $\mu^{\prime}\left(x_{1}, \ldots, x_{n}\right)=T_{L}\left(x_{1}, \ldots, x_{n}\right)$.

Coherence of $\left(x_{1}, \ldots, x_{n}, T_{M}\left(x_{1}, \ldots, x_{n}\right)\right)$. Without loss of generality we can assume that $x_{1} \leqslant x_{2} \leqslant \cdots \leqslant$ $x_{n}$. We show that the assessment $\left(x_{1}, \ldots, x_{n}, x_{1 \cdots n}\right)$, with $x_{1 \cdots n}=T_{M}\left(x_{1}, \ldots, x_{n}\right)=x_{1}$, is coherent. We simply observe that system $\left(\Sigma_{n}^{*}\right)$ in (20) is solvable with a solution $\Lambda_{n}=\left(\lambda_{i_{1} \cdots i_{n}} ;\left(i_{1}, \ldots, i_{n}\right) \in\{1, \overline{1}\} \times \cdots \times\right.$ $\{n, \bar{n}\})$ given by

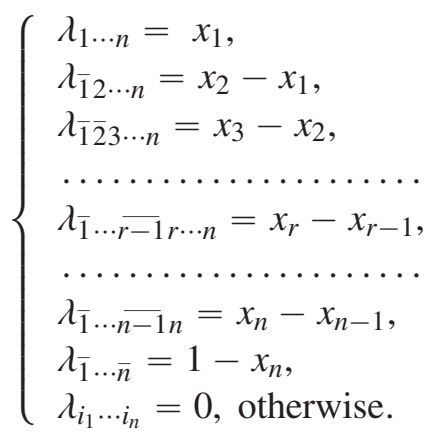


Indeed, based on (43), it holds that

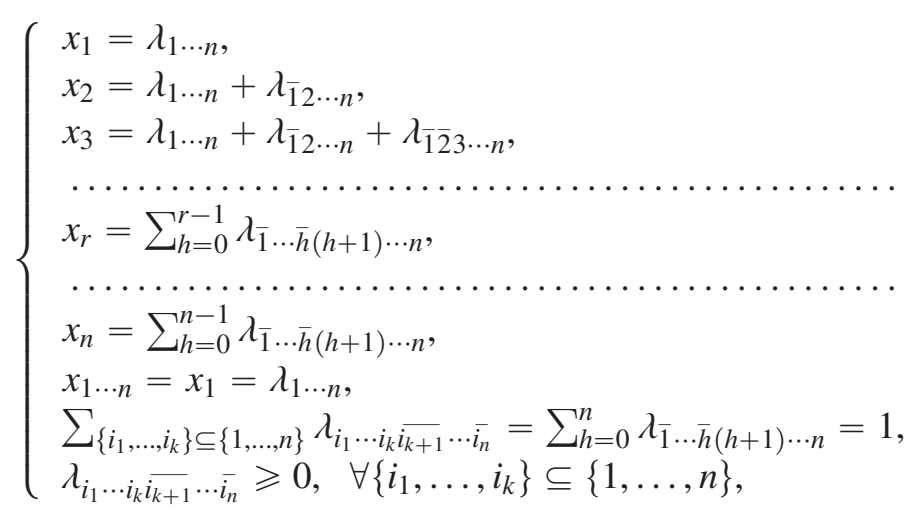

that is the system $\left(\Sigma_{n}^{*}\right)$ in (20) is solvable. Therefore, by Theorem 9 the assessment $\left(x_{1}, \ldots, x_{n}, x_{1 \ldots n}\right)$, with $x_{1 \cdots n}=T_{M}\left(x_{1}, \ldots, x_{n}\right)$, on $\mathcal{F}=\left\{E_{1}\left|H_{1}, \ldots, E_{n}\right| H_{n}, \mathcal{C}_{1 \ldots n}\right\}$ is coherent. Then, $\mu^{\prime \prime}\left(x_{1}, \ldots, x_{n}\right)=$ $T_{M}\left(x_{1}, \ldots, x_{n}\right)$.

Finally, the statement in (21) is valid.

\subsection{On the relationship between the sets $\Pi$ and $\mathcal{I}^{*}$}

In this section we show that the set $\Pi$ defined in (21) is convex and coincides with the set $I^{*}$ defined in (18). We first recall the properties of convexity and concavity of $T_{L}$ and $T_{M}$, respectively. Given two vectors $\mathcal{V}_{1}=\left(\alpha_{1}, \ldots, \alpha_{n}\right) \in[0,1]^{n}, \mathcal{V}_{2}=\left(\beta_{1}, \ldots, \beta_{n}\right) \in[0,1]^{n}$, and any quantity $a \in[0,1]$, we set

$$
\mathcal{V}=a \mathcal{V}_{1}+(1-a) \mathcal{V}_{2}=\left(\gamma_{1}, \ldots, \gamma_{n}\right) \text {. }
$$

Then, the following properties are satisfied:

$$
\begin{array}{cc}
(\text { convexity }) & T_{L}(\mathcal{V})=T_{L}\left(a \mathcal{V}_{1}+(1-a) \mathcal{V}_{2}\right) \leqslant a T_{L}\left(\mathcal{V}_{1}\right)+(1-a) T_{L}\left(\mathcal{V}_{2}\right), \\
(\text { concavity }) & a T_{M}\left(\mathcal{V}_{1}\right)+(1-a) T_{M}\left(\mathcal{V}_{2}\right) \leqslant T_{M}\left(a \mathcal{V}_{1}+(1-a) \mathcal{V}_{2}\right)=T_{M}(\mathcal{V})
\end{array}
$$

Convexity of $T_{L}$. We observe that

$$
\sum_{i=1}^{n}\left[a \alpha_{i}+(1-a) \beta_{i}\right]-(n-1)=a\left[\sum_{i=1}^{n} \alpha_{i}-(n-1)\right]+(1-a)\left[\sum_{i=1}^{n} \beta_{i}-(n-1)\right] .
$$

Then, we distinguish the following cases: $(i) T_{L}\left(\mathcal{V}_{1}\right) \geqslant 0, T_{L}\left(\mathcal{V}_{2}\right) \geqslant 0$, or $T_{L}\left(\mathcal{V}_{1}\right)<0, T_{L}\left(\mathcal{V}_{2}\right)<0$; $(i i)$ $T_{L}\left(\mathcal{V}_{1}\right) \geqslant 0, T_{L}\left(\mathcal{V}_{2}\right)<0$, or $T_{L}\left(\mathcal{V}_{1}\right)<0, T_{L}\left(\mathcal{V}_{2}\right) \geqslant 0$.

In case $(i)$ it holds that $T_{L}(\mathcal{V})=a T_{L}\left(\mathcal{V}_{1}\right)+(1-a) T_{L}\left(\mathcal{V}_{2}\right)$. In case $(i i)$ it holds that $T_{L}(\mathcal{V}) \leqslant a T_{L}\left(\mathcal{V}_{1}\right)+$ $(1-a) T_{L}\left(\mathcal{V}_{2}\right)$. Therefore, $T_{L}$ is convex.

Concavity of $T_{M}$. We observe that $T_{M}\left(\mathcal{V}_{1}\right)=\min \left\{\alpha_{1}, \ldots, \alpha_{n}\right\}$ and $T_{M}\left(\mathcal{V}_{2}\right)=\min \left\{\beta_{1}, \ldots, \beta_{n}\right\}=\beta^{*}$. We set $T_{M}\left(\mathcal{V}_{1}\right)=\alpha^{*}$ and $T_{M}\left(\mathcal{V}_{2}\right)=\beta^{*}$; moreover, we observe that

$$
a \alpha^{*}+(1-a) \beta^{*} \leqslant a \alpha_{i}+(1-a) \beta_{i}, i=1, \ldots, n,
$$

that is

$$
a \alpha^{*}+(1-a) \beta^{*} \leqslant \min \left\{a \alpha_{1}+(1-a) \beta_{1}, \ldots, a \alpha_{n}+(1-a) \beta_{n}\right\}=T_{M}(\mathcal{V})
$$

Then

$$
a T_{M}\left(\mathcal{V}_{1}\right)+(1-a)+(1-a) T_{M}\left(\mathcal{V}_{2}\right) \leqslant T_{M}(\mathcal{V})
$$

that is $T_{M}$ is concave.

In the next result we show that $\Pi$ is convex and coincide with $\mathcal{I}^{*}$. 
Theorem 11. Let $E_{1}, \ldots, E_{n}, H_{1}, \ldots, H_{n}$ be logically independents events, with $H_{1} \neq \varnothing, \ldots, H_{n} \neq \varnothing$, $n \geqslant 2$. The set $\Pi$ of all prevision coherent assessments $\mathcal{M}=\left(x_{1}, \ldots, x_{n}, x_{1 \ldots n}\right)$ on the family $\mathcal{F}=$ $\left\{E_{1}\left|H_{1}, \ldots, E_{n}\right| H_{n}, \mathcal{C}_{1 \ldots n}\right\}$ is convex and coincides with the set $\mathcal{I}^{*}$.

Proof. Let $\mathcal{M}_{1}=\left(\alpha_{1}, \ldots, \alpha_{n}, \alpha_{1 \ldots n}\right)$ and $\mathcal{M}_{2}=\left(\beta_{1}, \ldots, \beta_{n}, \beta_{1 \ldots n}\right)$ be two coherent assessments on $\mathcal{F}$. Given any $a \in[0,1]$ we show that the assessment

$$
\mathcal{M}=a \mathcal{M}_{1}+(1-a) \mathcal{M}_{2}=\left(\gamma_{1}, \ldots, \gamma_{n}, \gamma_{1 \cdots n}\right)
$$

on $\mathcal{F}$ is coherent. From $(21)$ it holds that $\left(\alpha_{1}, \ldots, \alpha_{n}\right) \in[0,1]^{n}$ and $\left(\beta_{1}, \ldots, \beta_{n}\right) \in[0,1]^{n}$. Then, $\left(\gamma_{1}, \ldots, \gamma_{n}\right) \in[0,1]^{n}$. Moreover,

$$
T_{L}\left(\alpha_{1}, \ldots, \alpha_{n}\right) \leqslant \alpha_{1 \ldots n} \leqslant T_{M}\left(\alpha_{1}, \ldots, \alpha_{n}\right), T_{L}\left(\beta_{1}, \ldots, \beta_{n}\right) \leqslant \beta_{1 \ldots n} \leqslant T_{M}\left(\beta_{1}, \ldots, \beta_{n}\right) .
$$

Then, by taking into account that $\gamma_{1 \cdots n}=a \alpha_{1 \cdots n}+(1-a) \beta_{1 \cdots n}$, it follows

$$
a T_{L}\left(\alpha_{1}, \ldots, \alpha_{n}\right)+(1-a) T_{L}\left(\beta_{1}, \ldots, \beta_{n}\right) \leqslant \gamma_{1 \cdots n} \leqslant a T_{M}\left(\alpha_{1}, \ldots, \alpha_{n}\right)+(1-a) T_{M}\left(\beta_{1}, \ldots, \beta_{n}\right) .
$$

By recalling (45) and (46), it holds that

$$
T_{L}\left(\gamma_{1}, \ldots, \gamma_{n}\right) \leqslant a T_{L}\left(\alpha_{1}, \ldots, \alpha_{n}\right)+(1-a) T_{L}\left(\beta_{1}, \ldots, \beta_{n}\right) \leqslant \gamma_{1 \cdots n} .
$$

and

$$
\gamma_{1 \cdots n} \leqslant a T_{M}\left(\alpha_{1}, \ldots, \alpha_{n}\right)+(1-a) T_{M}\left(\beta_{1}, \ldots, \beta_{n}\right) \leqslant T_{M}\left(\gamma_{1}, \ldots, \gamma_{n}\right) .
$$

Finally, by observing that

$$
\left(\gamma_{1}, \ldots, \gamma_{n}\right) \in[0,1]^{n}, \quad T_{L}\left(\gamma_{1}, \ldots, \gamma_{n}\right) \leqslant \gamma_{1 \cdots n} \leqslant T_{M}\left(\gamma_{1}, \ldots, \gamma_{n}\right),
$$

it follows that $\mathcal{M}$ is coherent, that is $\mathcal{M} \in \Pi$; thus $\Pi$ is convex.

We now show that $\Pi=I^{*}$. For each assessment $\mathcal{M}$ on $\mathcal{F}$, by Theorem 9 , if $\mathcal{M} \in \mathcal{I}^{*}$, then $\mathcal{M}$ is coherent. Thus $\mathcal{I}^{*} \subseteq \Pi$. Then, in order to complete the proof we need to show that $\Pi \subseteq \mathcal{I}^{*}$. Given any $\mathcal{M}=\left(x_{1}, \ldots, x_{n}, x_{1 \ldots n}\right) \in \Pi$, by Theorem 10 it holds that $x_{1 \cdots n} \in\left[T_{L}\left(x_{1}, \ldots, x_{n}\right), T_{M}\left(x_{1}, \ldots, x_{n}\right)\right]$. Then, there exists $\alpha \in[0,1]$ such that

$$
x_{1 \cdots n}=\alpha T_{L}\left(x_{1}, \ldots, x_{n}\right)+(1-\alpha) T_{M}\left(x_{1}, \ldots, x_{n}\right),
$$

and hence

$$
\mathcal{M}=\left(x_{1}, \ldots, x_{n}, x_{1 \ldots n}\right)=\alpha\left(x_{1}, \ldots, x_{n}, T_{L}\left(x_{1}, \ldots, x_{n}\right)\right)+(1-\alpha)\left(x_{1}, \ldots, x_{n}, T_{M}\left(x_{1}, \ldots, x_{n}\right)\right) .
$$

We denote by $\Lambda_{L}$, or $\Lambda_{M}$, a solution of the system $\left(\Sigma_{n}^{*}\right)$ associated with the assessment $\left(x_{1}, \ldots, x_{n}, T_{L}\left(x_{1}, \ldots, x_{n}\right)\right)$, or the assessment $\left(x_{1}, \ldots, x_{n}, T_{M}\left(x_{1}, \ldots, x_{n}\right)\right)$, respectively. Then, the vector $\Lambda=\alpha \Lambda_{L}+(1-\alpha) \Lambda_{M}$ is a solution of the system $\left(\Sigma_{n}^{*}\right)$ associated with the assessment $\mathcal{M}=$ $\left(x_{1}, \ldots, x_{n}, x_{1 \ldots n}\right)$; thus $\mathcal{M} \in \mathcal{I}^{*}$, so that $\Pi \subseteq \mathcal{I}^{*}$. Therefore $\mathcal{I}^{*}=\Pi$. 


\subsection{An illustration of sharpness of the Fréchet-Hoeffding bounds in the case of three conditional events}

In this section, to better understand the previous general results, we illustrate some details which concern the case of three conditional events. Given any logically independent events $E_{1}, E_{2}, E_{3}, H_{1}, H_{2}, H_{3}$, let $\mathcal{M}=\left(x_{1}, x_{2}, x_{3}, x_{123}\right)$ be a prevision assessment on $\mathcal{F}=\left\{E_{1}\left|H_{1}, E_{2}\right| H_{2}, E_{3} \mid H_{3}, \mathcal{C}_{123}\right\}$. The constituents $\mathrm{C}_{h}$ 's which imply $\mathrm{H}_{1} \mathrm{H}_{2} \mathrm{H}_{3}$ are

$$
\begin{aligned}
& K_{123}=E_{1} E_{2} E_{3} H_{1} H_{2} H_{3}, K_{1 \overline{2} 3}=E_{1} \bar{E}_{2} E_{3} H_{1} H_{2} H_{3}, K_{\overline{1} 23}=\bar{E}_{1} E_{2} E_{3} H_{1} H_{2} H_{3}, K_{\overline{1} \overline{2} 3}=\bar{E}_{1} \bar{E}_{2} E_{3} H_{1} H_{2} H_{3}, \\
& K_{12 \overline{3}}=E_{1} E_{2} \bar{E}_{3} H_{1} H_{2} H_{3}, K_{1 \overline{2} \overline{3}}=E_{1} \bar{E}_{2} \bar{E}_{3} H_{1} H_{2} H_{3}, K_{\overline{1} 2 \overline{3}}=\bar{E}_{1} E_{2} \bar{E}_{3} H_{1} H_{2} H_{3}, K_{\overline{1} \overline{2} \overline{3}}=\bar{E}_{1} \bar{E}_{2} \bar{E}_{3} H_{1} H_{2} H_{3} .
\end{aligned}
$$

The associated points $Q_{123}, \ldots, Q_{\overline{1} \overline{2} \overline{3}}$ are

$$
\begin{aligned}
& Q_{123}=(1,1,1,1), Q_{1 \overline{3} 3}=(1,0,1,0), Q_{\overline{1} 23}=(0,1,1,0), Q_{\overline{1} \overline{2} 3}=(0,0,1,0), \\
& Q_{12 \overline{3}}=(1,1,0,0), Q_{1 \overline{3} \overline{3}}=(1,0,0,0), Q_{\overline{1} 2 \overline{3}}=(0,1,0,0), Q_{\overline{1} \overline{2} \overline{3}}=(0,0,0,0) .
\end{aligned}
$$

In this case system $\left(\Sigma_{n}^{*}\right)$ in 20 becomes

$$
\left(\Sigma_{3}^{*}\right)\left\{\begin{array}{l}
x_{123}=\lambda_{123} \\
x_{1}=\lambda_{123}+\lambda_{1 \overline{2} 3}+\lambda_{12 \overline{3}}+\lambda_{1 \overline{2} \overline{3}} \\
x_{2}=\lambda_{123}+\lambda_{\overline{1} 23}+\lambda_{12 \overline{3}}+\lambda_{\overline{1} 2 \overline{3}} \\
x_{3}=\lambda_{123}+\lambda_{1 \overline{2} 3}+\lambda_{\overline{1} 23}+\lambda_{\overline{1} \overline{2} 3}, \\
\lambda_{123}+\cdots+\lambda_{1 \overline{2} \overline{3}}=1, \quad \lambda_{123} \geqslant 0, \ldots, \lambda_{\overline{1} \overline{2} \overline{3}} \geqslant 0 .
\end{array}\right.
$$

By recalling the proof of Theorem (10), we illustrate below the structure of the vector $\Lambda_{3}=$ $\left(\lambda_{123}, \lambda_{12}{ }_{3}, \lambda_{123}, \lambda_{\overline{1} \overline{2} 3}, \lambda_{12 \overline{3}}, \lambda_{1 \overline{2} \overline{3}}, \lambda_{\overline{1} 2 \overline{3}}, \lambda_{\overline{1} \overline{2} \overline{3}}\right)$, solution of $\left(\Sigma_{3}^{*}\right)$, in the different cases.

Assessment $\left(x_{1}, x_{2}, x_{3}, x_{123}\right)$, with $x_{123}=T_{L}\left(x_{1}, x_{2}, x_{3}\right)$.

We have two cases: $(i) T_{L}\left(x_{1}, x_{2}\right)=0$, that is $x_{1}+x_{2}-1 \leqslant 0$; (ii) $T_{L}\left(x_{1}, x_{2}\right)>0$, that is $x_{1}+x_{2}-1>0$. In case $(i) T_{L}\left(x_{1}, x_{2}\right)=T_{L}\left(x_{1}, x_{2}, x_{3}\right)=0$ and we have three sub-cases: $x_{3}=0$, or $x_{3}=1$, or $0<x_{3}<1$. If $x_{3}=0$ the system $\left(\Sigma_{3}^{*}\right)$ has a solution

$$
\Lambda_{3,0}=\left(0,0,0,0,0, x_{1}, x_{2}, 1-x_{1}-x_{2}\right) .
$$

If $x_{3}=1$ the system $\left(\Sigma_{3}^{*}\right)$ has a solution

$$
\Lambda_{3,1}=\left(0, x_{1}, x_{2}, 1-x_{1}-x_{2}, 0,0,0,0\right) .
$$

If $0<x_{3}<1$ the system $\left(\Sigma_{3}^{*}\right)$ has a solution

$$
\begin{aligned}
& \Lambda_{3}=\left(1-x_{3}\right) \Lambda_{3,0}+x_{3} \Lambda_{3,1}= \\
& =\left(0, x_{1} x_{3}, x_{2} x_{3},\left(1-x_{1}-x_{2}\right) x_{3}, 0, x_{1}\left(1-x_{3}\right), x_{2}\left(1-x_{3}\right),\left(1-x_{1}-x_{2}\right)\left(1-x_{3}\right)\right) .
\end{aligned}
$$

In case $(i i)$, where $T_{L}\left(x_{1}, x_{2}\right)>0$, we have two sub-cases:

(ii.a) $0 \leqslant x_{3} \leqslant 2-x_{1}-x_{2}<1$; (ii.b) $2-x_{1}-x_{2}<x_{3} \leqslant 1$.

Sub-case (ii.a). We have three cases:

(ii.a.1) $x_{3}=0$; (ii.a.2) $x_{3}=2-x_{1}-x_{2}$; (ii.a.3) $0<x_{3}<2-x_{1}-x_{2}$.

If $x_{3}=0$ the system $\left(\Sigma_{3}^{*}\right)$ has a solution

$$
\Lambda_{3,0}=\left(0,0,0,0, x_{1}+x_{2}-1,1-x_{2}, 1-x_{1}, 0\right) .
$$


If $x_{3}=2-x_{1}-x_{2}=0$, a solution of $\left(\Sigma_{3}^{*}\right)$ is the vector in (48).

If $x_{3}=2-x_{1}-x_{2}>0$, by setting $s=2-x_{1}-x_{2}$, the system $\left(\Sigma_{3}^{*}\right)$ has a solution

$$
\Lambda_{3, s}=\left(0,1-x_{2}, 1-x_{1}, 0, x_{1}+x_{2}-1,0,0,0\right) .
$$

If $0<x_{3}<2-x_{1}-x_{2}$ the system $\left(\Sigma_{3}^{*}\right)$ has a solution

$$
\begin{aligned}
& \Lambda_{3}=\left(1-\frac{x_{3}}{2-x_{1}-x_{2}}\right) \Lambda_{3,0}+\frac{x_{3}}{2-x_{1}-x_{2}} \Lambda_{3, s}= \\
& =\left(0, \frac{\left(1-x_{2}\right) x_{3}}{2-x_{1}-x_{2}}, \frac{\left(1-x_{1}\right) x_{3}}{2-x_{1}-x_{2}}, 0, x_{1}+x_{2}-1,\left(1-\frac{x_{3}}{2-x_{1}-x_{2}}\right)\left(1-x_{2}\right),\left(1-\frac{x_{3}}{2-x_{1}-x_{2}}\right)\left(1-x_{1}\right), 0\right) .
\end{aligned}
$$

Sub-case (ii.b). The system $\left(\Sigma_{3}^{*}\right)$ has a solution

$$
\Lambda_{3}=\left(x_{1}+x_{2}+x_{3}-2,1-x_{2}, 1-x_{1}, 0,1-x_{3}, 0,0,0\right) .
$$

Assessment $\left(x_{1}, x_{2}, x_{3}, x_{123}\right)$, with $x_{123}=T_{M}\left(x_{1}, x_{2}, x_{3}\right)$. We assume that $0 \leqslant x_{1} \leqslant x_{2} \leqslant x_{3} \leqslant 1$, so that $x_{123}=T_{M}\left(x_{1}, x_{2}, x_{3}\right)=x_{1}$. The system $\left(\Sigma_{3}^{*}\right)$ has a solution

$$
\Lambda_{3}=\left(\lambda_{123}, \lambda_{1 \overline{2} 3}, \lambda_{\overline{1} 23}, \lambda_{\overline{1} \overline{2} 3}, \lambda_{12 \overline{3}}, \lambda_{1 \overline{2} \overline{3}}, \lambda_{\overline{1} 2 \overline{3}}, \lambda_{\overline{1} \overline{3}}\right)=\left(x_{1}, 0, x_{2}-x_{1}, x_{3}-x_{2}, 0,0,0,1-x_{3}\right) .
$$

We give below two examples.

Example 3. Let $\left(x_{1}, x_{2}, x_{3}, T_{L}\left(x_{1}, x_{2}, x_{3}\right)\right)=(0.4,0.4,0.4,0)$ be a prevision assessment on $\mathcal{F}=$ $\left\{E_{1}\left|H_{1}, E_{2}\right| H_{2}, E_{3} \mid H_{3}, \mathcal{C}_{123}\right\}$. We observe that $x_{1}+x_{2}-1=-0.2<0$; then, based on (477), the system $\left(\Sigma_{3}^{*}\right)$ has a solution

$$
\Lambda_{3}=\left(\lambda_{123}, \lambda_{1 \overline{2} 3}, \lambda_{\overline{1} 23}, \lambda_{\overline{1} \overline{2} 3}, \lambda_{12 \overline{3}}, \lambda_{1 \overline{2} \overline{3}}, \lambda_{\overline{1} 2 \overline{3}}, \lambda_{\overline{1} \overline{2} \overline{3}}\right)=(0,0.16,0.16,0.08,0,0.24,0.24,0.12) .
$$

Example 4. Let $\left(x_{1}, x_{2}, x_{3}, T_{L}\left(x_{1}, x_{2}, x_{3}\right)\right)=(0.5,0.6,0.7,0)$ be a prevision assessment on $\mathcal{F}=$ $\left\{E_{1}\left|H_{1}, E_{2}\right| H_{2}, E_{3} \mid H_{3}, \mathcal{C}_{123}\right\}$. As $x_{1}+x_{2}-1=0.1>0$, based on (49), the system $\left(\Sigma_{3}^{*}\right)$ has a solution

$$
\Lambda_{3}=\left(\lambda_{123}, \lambda_{1 \overline{2} 3}, \lambda_{\overline{1} 23}, \lambda_{\overline{1} \overline{2} 3}, \lambda_{12 \overline{3}}, \lambda_{1 \overline{2} \overline{3}}, \lambda_{\overline{1} 2 \overline{3}}, \lambda_{\overline{1} \overline{2} \overline{3}}\right)=\left(0, \frac{14}{45}, \frac{7}{18}, 0, \frac{1}{10}, \frac{4}{45}, \frac{1}{9}, 0\right) .
$$

\section{On the computation of $\Lambda_{n+1}$ when $x_{1 \cdots n+1}=T_{L}\left(x_{1}, \ldots, x_{n+1}\right)$}

In this section we examine further aspects which concern the prevision assessment $\left(x_{1}, \ldots, x_{n+1}, T_{L}\left(x_{1}, \ldots, x_{n+1}\right)\right)$. We observe that in the proof of Theorem 10 the explicit solution $\Lambda_{n+1}$ of the system $\left(\Sigma_{n+1}^{*}\right)$ is given for the assessment $\left(x_{1}, \ldots, x_{n+1}, T_{L}\left(x_{1}, \ldots, x_{n+1}\right)\right.$, when $T_{L}\left(x_{1}, \ldots, x_{n}\right)>0$. In the case where $T_{L}\left(x_{1}, \ldots, x_{n}\right)=0$, for the vector $\Lambda_{n+1}$ we only have the representation given in (30) in terms of the solution $\Lambda_{n}$. In what follows we give an explicit formula for $\Lambda_{n+1}$ when $T_{L}\left(x_{1}, \ldots, x_{n}\right)=0$.

Given any integer $n \geqslant 1$, we distinguish two cases: $(i) T_{L}\left(x_{1}, \ldots, x_{h}\right)=0$, for all $h=1, \ldots, n$; (ii) $T_{L}\left(x_{1}\right)>0, \ldots, T_{L}\left(x_{1}, \ldots, x_{h}\right)>0, T_{L}\left(x_{1}, \ldots, x_{h+1}\right)=\cdots=T_{L}\left(x_{1}, \ldots, x_{n}\right)=0$, for some $h$ such that $1 \leqslant h<n$.

Case $(i)$. If $n=1$, the assessment is $\left(x_{1}, x_{2}, T_{L}\left(x_{1}, x_{2}\right)\right)$ and $T_{L}\left(x_{1}\right)=x_{1}=0$. Moreover, the (unique) solution of $\left(\Sigma_{1}^{*}\right)$ is $\Lambda_{1}=\left(\lambda_{1}, \lambda_{\overline{1}}\right)=\left(x_{1}, 1-x_{1}\right)=(0,1)$. Then, by applying (30) with $n=1$, we obtain the solution

$$
\Lambda_{2}=\left(\lambda_{12}, \lambda_{\overline{1} 2}, \lambda_{1 \overline{2}}, \lambda_{\overline{1} \overline{2}}\right)=\left(x_{2} \Lambda_{1},\left(1-x_{2}\right) \Lambda_{1}\right)=\left(x_{2}(0,1),\left(1-x_{2}\right)(0,1)\right)=\left(0, x_{2}, 0,1-x_{2}\right) .
$$


If $n=2$, the assessment is $\left(x_{1}, x_{2}, x_{3}, T_{L}\left(x_{1}, x_{2}, x_{3}\right)\right)$ and $T_{L}\left(x_{1}\right)=x_{1}=T_{L}\left(x_{1}, x_{2}\right)=0$. Moreover, as $x_{1}=0$, it holds that $\Lambda_{2}$ is the vector given in (50) and by applying (30) with $n=2$, a solution for $\left(\Sigma_{3}^{*}\right)$ is given by

$$
\begin{aligned}
& \Lambda_{3}=\left(\lambda_{123}, \lambda_{\overline{1} 23}, \lambda_{1 \overline{2} 3}, \lambda_{\overline{1} \overline{2} 3}, \lambda_{12 \overline{3}}, \lambda_{\overline{1} 2 \overline{3}}, \lambda_{1 \overline{2} \overline{3}}, \lambda_{\overline{1} \overline{2} \overline{3}}\right)=\left(x_{3} \Lambda_{2},\left(1-x_{3}\right) \Lambda_{2}\right)= \\
& =\left(0, x_{2} x_{3}, 0,\left(1-x_{2}\right) x_{3}, 0, x_{2}\left(1-x_{3}\right), 0,\left(1-x_{2}\right)\left(1-x_{3}\right)\right) .
\end{aligned}
$$

More in general, by iterating (30) we obtain

$$
\begin{aligned}
& \Lambda_{n+1}=\left(\lambda_{1 \cdots n+1}, \ldots, \lambda_{\overline{1} \cdots \bar{n} n+1}, \lambda_{1 \cdots n n+1}, \ldots, \lambda_{\overline{1} \cdots \bar{n} \overline{n+1}}\right)= \\
& =\left(x_{1} \cdots x_{n} x_{n+1}, \ldots,\left(1-x_{1}\right) \cdots\left(1-x_{n}\right) x_{n+1}, x_{1} \cdots x_{n}\left(1-x_{n+1}\right), \ldots,\left(1-x_{1}\right) \cdots\left(1-x_{n}\right)\left(1-x_{n+1}\right)\right),
\end{aligned}
$$

where $x_{1}=0$. Alternatively, by setting

$$
\Lambda_{n+1}=\left(\lambda_{1 * \ldots(n+1) *} ;\left(1^{*}, \ldots,(n+1)^{*}\right) \in\{1, \overline{1}\} \times \cdots \times\{n+1, \overline{n+1}\}\right),
$$

and

$$
x_{j^{*}}=\left\{\begin{array}{l}
x_{j}, \text { if } j^{*}=j \\
x_{\bar{j}}=1-x_{j}, \text { if } j^{*}=\bar{j}
\end{array}\right.
$$

it holds that

$$
\lambda_{1 * \ldots(n+1)^{*}}=\prod_{j=1}^{n+1} x_{j^{*}}, \quad\left(1^{*}, \ldots,(n+1)^{*}\right) \in\{1, \overline{1}\} \times \cdots \times\{n+1, \overline{n+1}\},
$$

where $\prod_{j=1}^{n+1} x_{j^{*}}=0$, if $1^{*}=1$, because $x_{1}=0$, and $\prod_{j=1}^{n+1} x_{j^{*}}=\prod_{j=2}^{n+1} x_{j^{*}}$, if $1^{*}=\overline{1}$, because $x_{\overline{1}}=1-x_{1}=1$.

Case (ii). For $t=h+1, \ldots, n$, it holds that $T_{L}\left(x_{1}, \ldots, x_{t+1}\right)=0$ and, from (30), it holds that

$$
\begin{aligned}
& \Lambda_{t+1}=\left(\lambda_{1 \cdots t+1}, \ldots, \lambda_{\overline{1} \cdots \bar{t} t+1}, \lambda_{1 \cdots t \overline{t+1}}, \ldots, \lambda_{\overline{1} \cdots \bar{t} \overline{t+1}}\right)= \\
& =\left(x_{t+1} \lambda_{1 \cdots t}, \ldots, x_{t+1} \lambda_{\overline{1} \cdots \bar{t}},\left(1-x_{t+1}\right) \lambda_{1 \cdots t}, \ldots,\left(1-x_{t+1}\right) \lambda_{\overline{1} \ldots \bar{t}}\right) .
\end{aligned}
$$

Then, based on the representations

$$
\Lambda_{t}=\left(\lambda_{1 * \ldots t^{*}} ;\left(1^{*}, \ldots, t^{*}\right) \in\{1, \overline{1}\} \times \cdots \times\{t, \bar{t}\}\right),
$$

and

$$
\Lambda_{t+1}=\left(\lambda_{1 * \ldots(t+1)^{*}} ;\left(1^{*}, \ldots,(t+1)^{*}\right) \in\{1, \overline{1}\} \times \cdots \times\{t+1, \overline{t+1}\}\right),
$$

based on (51), for the components $\lambda_{1 * \ldots(t+1) *}$ and $\lambda_{1 * \ldots t *}$ it holds that

$$
\lambda_{1} * \ldots(t+1) *=\lambda_{1 * \ldots t^{*}} \cdot x_{(t+1) *},
$$

that is

$$
\left\{\begin{array}{l}
\lambda_{1 * \ldots t^{*} t+1}=\lambda_{1 * \ldots t^{*}} \cdot x_{t+1}, \\
\lambda_{1 * \ldots t * \overline{t+1}}=\lambda_{1 * \ldots t^{*}} \cdot\left(1-x_{t+1}\right),
\end{array}\right.
$$

for every $\left(1^{*}, \ldots, t^{*}\right) \in\{1, \overline{1}\} \times \cdots \times\{t, \bar{t}\}, t=h+1, \ldots, n$.

By iterating (53) backward from $t=n$ until $t=h+1$, it follows that

$$
\lambda_{1 * \ldots(n+1) *}=\lambda_{1 * \ldots n^{*}} \cdot x_{(n+1) *}=\lambda_{1 * \ldots(n-1) *} \cdot x_{n} * x_{(n+1)}=\cdots=\lambda_{1 * \ldots(h+1)} * \prod_{t=h+2}^{n+1} x_{t^{*}} .
$$


Thus, in order to determine the vector $\Lambda_{n+1}$ we need to compute the vector $\Lambda_{h+1}$. We examine below this aspect.

If $0<T_{L}\left(x_{1}, \ldots, x_{h}\right)<1$, as $T_{L}\left(x_{1}, \ldots, x_{h+1}\right)=0$ it holds that $0 \leqslant x_{h+1} \leqslant h-x_{1}-\cdots-x_{h}$; then from (41) we obtain

$$
\left\{\begin{array}{l}
\lambda_{1 \cdots h+1}=0, \\
\lambda_{1 \cdots r-1} \bar{r} r+1 \cdots h+1=\frac{x_{h+1}}{h-\left(x_{1}+\cdots+x_{h}\right)}\left(1-x_{r}\right), \quad r=1, \ldots, h, \\
\lambda_{i_{1} \cdots i_{k}} \overline{i_{k+1} \cdots \bar{i}_{h} h+1}=0, \forall\left\{i_{1}, \ldots, i_{k}\right\} \subseteq\{1, \ldots, h\}, \quad k<h-1, \\
\lambda_{1 \cdots h h+1}=x_{1}+\cdots+x_{h}-h+1, \\
\lambda_{1 \cdots r-1} \bar{r} r+1 \cdots h \overline{h+1}=\left(1-\frac{x_{h+1}}{h-\left(x_{1}+\cdots+x_{h}\right)}\right)\left(1-x_{r}\right), \quad r=1, \ldots, h, \\
\lambda_{i_{1} \cdots i_{k} \overline{i_{k+1}} \cdots \bar{i}_{h} \overline{h+1}}=0, \forall\left\{i_{1}, \ldots, i_{k}\right\} \subseteq\{1, \ldots, h\}, \quad k<h-1 .
\end{array}\right.
$$

Then, concerning the components of $\Lambda_{n+1}$, for every

$$
\left((h+2)^{*}, \ldots,(n+1)^{*}\right) \in\{h+2, \overline{h+2}\} \times \cdots \times\{n+2, \overline{n+2}\},
$$

from (54) and (55) it follows that

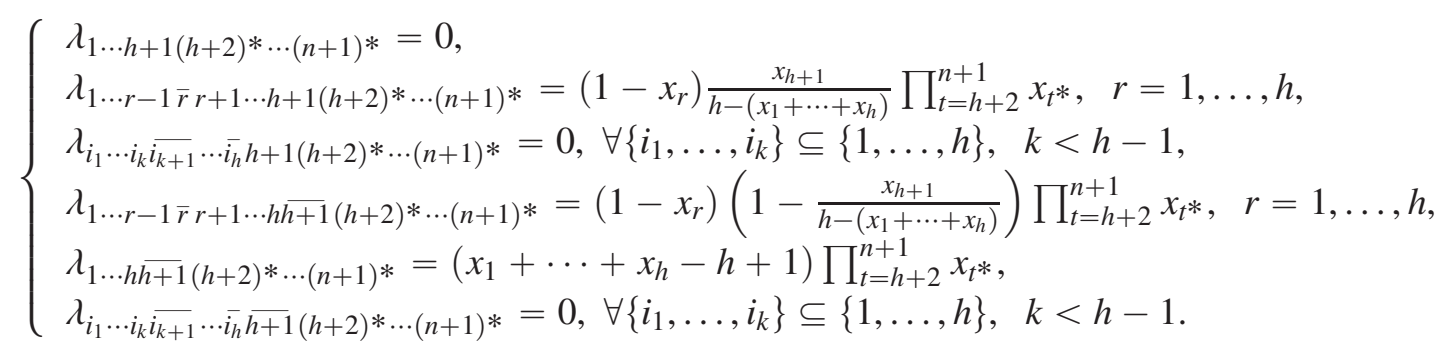

Of course the sum of all the components of $\Lambda_{n+1}$ is equal to 1 , indeed

$$
\begin{gathered}
\left(1-x_{r}\right) \frac{x_{h+1}}{h-\left(x_{1}+\cdots+x_{h}\right)} \prod_{t=h+2}^{n+1} x_{t^{*}}+\left(1-x_{r}\right)\left(1-\frac{x_{h+1}}{h-\left(x_{1}+\cdots+x_{h}\right)}\right) \prod_{t=h+2}^{n+1}=\left(1-x_{r}\right) \prod_{t=h+2}^{n+1} x_{t^{*}}, \\
\sum_{r=1}^{h}\left(1-x_{r}\right) \prod_{t=h+2}^{n+1} x_{t^{*}}=\left(h-\left(x_{1}+\cdots+x_{h}\right)\right) \prod_{t=h+2}^{n+1} x_{t^{*}}, \\
\left(x_{1}+\cdots+x_{h}-h+1\right) \prod_{t=h+2}^{n+1} x_{t^{*}}+\left(h-\left(x_{1}+\cdots+x_{h}\right)\right) \prod_{t=h+2}^{n+1} x_{t^{*}}=\prod_{t=h+2}^{n+1} x_{t^{*}} ;
\end{gathered}
$$

finally

$$
\begin{aligned}
& \sum_{((h+2) *, \ldots,(n+1) *)} \prod_{t=h+2}^{n+1} x_{t^{*}}=\sum_{\left((h+2) *, \ldots, n^{*}\right)} \prod_{t=h+2}^{n} x_{t} *\left(x_{n+1}+1-x_{n+1}\right)= \\
& =\sum_{\left((h+2) *, \ldots, n^{*}\right)} \prod_{t=h+2}^{n} x_{t^{*}}=\ldots=x_{h+2}+\left(1-x_{h+2}\right)=1 .
\end{aligned}
$$

If $T_{L}\left(x_{1}, \ldots, x_{h}\right)=1$, as $T_{L}\left(x_{1}, \ldots, x_{h+1}\right)=0$ it holds that $x_{h+1}=0$; then, concerning the vector $\Lambda_{h+1}$, from (37) we obtain that

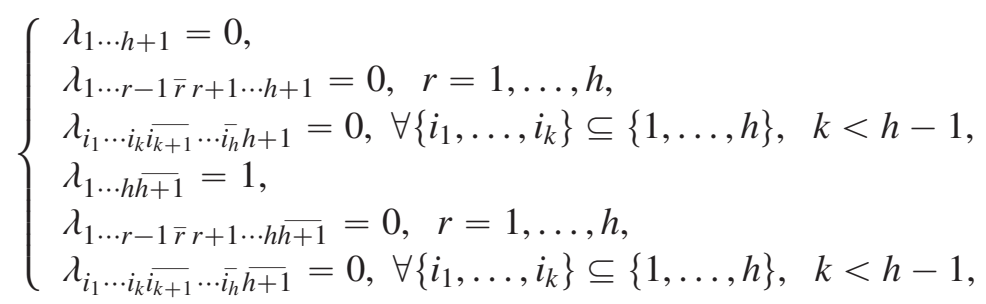


that is the vector $\Lambda_{h+1}$ has the component $\lambda_{1 \ldots h \overline{h+1}}$ equal to 1 and all the other components equal to zero. Then, concerning the components of $\Lambda_{n+1}$, for every

$$
\left((h+2)^{*}, \ldots,(n+1)^{*}\right) \in\{h+2, \overline{h+2}\} \times \cdots \times\{n+2, \overline{n+2}\},
$$

from (54) and (57) it follows that

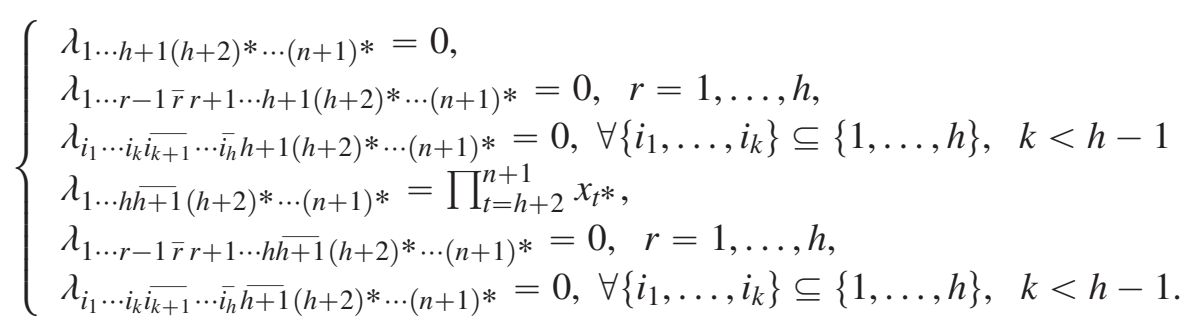

Remark 4. In summary, concerning the problem of giving an explicit solution $\Lambda_{n+1}$ of the system $\left(\Sigma_{n+1}^{*}\right)$ for the assessment $\left(x_{1}, \ldots, x_{n+1}, T_{L}\left(x_{1}, \ldots, x_{n+1}\right)\right.$, we distinguish the following cases:

(a) $T_{L}\left(x_{1}, \ldots, x_{n}\right)=1$ and $T_{L}\left(x_{1}, \ldots, x_{n+1}\right)=0$ (in which case $x_{n+1}=n-\sum_{i=1}^{n} x_{i}=0$ ); the solution is given in (37).

(b) $0<T_{L}\left(x_{1}, \ldots, x_{n}\right)<1$ and $T_{L}\left(x_{1}, \ldots, x_{n+1}\right)=0$ (in which case $0 \leqslant x_{n+1} \leqslant n-\sum_{i=1}^{n} x_{i}$, with $\left.0<n-\sum_{i=1}^{n} x_{i}<1\right)$; the solution is given in (41).

(c) $T_{L}\left(x_{1}, \ldots, x_{n}\right)>0$ and $T_{L}\left(x_{1}, \ldots, x_{n}+1\right)>0$ (in which case $n-\sum_{i=1}^{n} x_{i}<x_{n+1} \leqslant 1$ ); the solution is given in (42).

(d) $T_{L}\left(x_{1}, \ldots, x_{h}\right)=0, h=1, \ldots, n+1$ (in which case $x_{1}=0$ ); the solution is given in (52).

(e) $0<T_{L}\left(x_{1}, \ldots, x_{h}\right)<1, T_{L}\left(x_{1}, \ldots, x_{h+1}\right)=\cdots=T_{L}\left(x_{1}, \ldots, x_{n+1}\right)=0$, with $1 \leqslant h<n$; the solution is given in (56).

(f) $T_{L}\left(x_{1}, \ldots, x_{h}\right)=1, T_{L}\left(x_{1}, \ldots, x_{h+1}\right)=\cdots=T_{L}\left(x_{1}, \ldots, x_{n+1}\right)=0$, with $1 \leqslant h<n$; the solution is given in (58).

We illustrate below the cases $(e)$ and $(f)$ by an example where $n+1=5$.

Example 5. Given any logically independent events. Let $E_{1}, \ldots, E_{5}, H_{1}, \ldots, H_{5}$ be logically Let $\left(x_{1}, \ldots, x_{5}, x_{1 \ldots 5}\right)$, with $x_{1 \ldots 5}=T_{L}\left(x_{1}, \ldots, x_{5}\right)$, be a prevision assessment on $\left\{E_{1}\left|H_{1}, \ldots, E_{5}\right| H_{5}, \mathcal{C}_{1 \ldots 5}\right\}$, where $\mathcal{C}_{1 \ldots 5}=\bigwedge_{i=1}^{5} E_{i} \mid H_{i}$. We examine below all the cases of Remark 4

(a) $T_{L}\left(x_{1}, \ldots, x_{4}\right)=1$ and $T_{L}\left(x_{1}, \ldots, x_{5}\right)=0$ (in which case $x_{1}=\cdots=x_{4}=1$ and $x_{5}=4-\sum_{i=1}^{4} x_{i}=$ 0 ); the solution obtained from (37) is such that $\lambda_{1234 \overline{5}}=1$, with all the other components of $\Lambda_{5}$ equal to zero.

(b) $0<T_{L}\left(x_{1}, \ldots, x_{4}\right)<1$ and $T_{L}\left(x_{1}, \ldots, x_{5}\right)=0$ (in which case $0 \leqslant x_{5} \leqslant 4-\sum_{i=1}^{4} x_{i}$, with $\left.0<4-\sum_{i=1}^{4} x_{i}<1\right)$; the solution obtained from (41) is

$$
\begin{cases}\lambda_{\overline{1} 2345}=\frac{x_{5}}{4-\left(x_{1}+x_{2}+x_{3}+x_{4}\right)}\left(1-x_{1}\right), & \lambda_{\overline{1} 234 \overline{5}}=\left(1-\frac{x_{5}}{4-\left(x_{1}+x_{2}+x_{3}+x_{4}\right)}\right)\left(1-x_{1}\right), \\ \lambda_{1 \overline{2} 345}=\frac{x_{5}}{4-\left(x_{1}+x_{2}+x_{3}+x_{4}\right)}\left(1-x_{2}\right), & \lambda_{1 \overline{2} 34 \overline{5}}=\left(1-\frac{x_{5}}{4-\left(x_{1}+x_{2}+x_{3}+x_{4}\right)}\right)\left(1-x_{2}\right), \\ \lambda_{12 \overline{3} 45}=\frac{x_{5}}{4-\left(x_{1}+x_{2}+x_{3}+x_{4}\right)}\left(1-x_{3}\right), & \lambda_{12 \overline{3} 4 \overline{5}}=\left(1-\frac{x_{5}}{4-\left(x_{1}+x_{2}+x_{3}+x_{4}\right)}\right)\left(1-x_{3}\right), \\ \lambda_{123 \overline{4} 5}=\frac{x_{5}}{4-\left(x_{1}+x_{2}+x_{3}+x_{4}\right)}\left(1-x_{4}\right), & \lambda_{123 \overline{5}}=\left(1-\frac{x_{4}}{4-\left(x_{1}+x_{2}+x_{3}+x_{4}\right)}\right)\left(1-x_{4}\right), \\ \lambda_{1234 \overline{5}}=x_{1}+x_{2}+x_{3}+x_{4}-3, & \lambda_{1} * 2 * 3 * 4 * 5 *=0, \text { otherwise. }\end{cases}
$$


(c) $T_{L}\left(x_{1}, \ldots, x_{4}\right)>0$ and $T_{L}\left(x_{1}, \ldots, x_{5}\right)>0$ (in which case $4-\sum_{i=1}^{4} x_{i}<x_{5} \leqslant 1$ ); the solution obtained from (42) is

$$
\left\{\begin{array}{l}
\lambda_{12345}=x_{1}+x_{2}+x_{3}+x_{4}+x_{5}-4 \\
\lambda_{12345}=1-x_{1}, \quad \lambda_{1} \overline{2}_{345}=1-x_{2} \\
\lambda_{12 \overline{3} 45}=1-x_{3}, \quad \lambda_{123 \overline{4} 5}=1-x_{4} \\
\lambda_{1234 \overline{5}}=1-x_{5}, \quad \lambda_{1 * 2 * 3 * 4 * 5 *}=0, \text { otherwise. }
\end{array}\right.
$$

(d) $T_{L}\left(x_{1}\right)=\cdots=T_{L}\left(x_{1}, x_{2}, x_{3}, x_{4}, x_{5}\right)=0$ (in which case $x_{1}=0$ ); the solution obtained from (52) is

$$
\begin{aligned}
& \lambda_{12345}=0, \\
& \lambda_{\overline{1} 2345}=x_{2} x_{3} x_{4} x_{5}, \\
& \lambda_{1 \overline{2} 345}=0 \text {, } \\
& \lambda_{1 \overline{2} 345}=\left(1-x_{2}\right) x_{3} x_{4} x_{5}, \\
& \lambda_{12 \overline{3} 45}=0 \text {, } \\
& \lambda_{\overline{1} 2 \overline{3} 45}=x_{2}\left(1-x_{3}\right) x_{4} x_{5}, \\
& \lambda_{1 \overline{2} \overline{3} 45}=0 \text {, } \\
& \lambda_{1 \overline{2} \overline{3} 45}=\left(1-x_{2}\right)\left(1-x_{3}\right) x_{4} x_{5}, \\
& \lambda_{123 \overline{4} 5}=0 \text {, } \\
& \lambda_{\overline{1} 23 \overline{4} 5}=x_{2} x_{3}\left(1-x_{4}\right) x_{5} \text {, } \\
& \lambda_{1 \overline{2} 3 \overline{4} 5}=0 \text {, } \\
& \lambda_{\overline{1} \overline{2} 3 \overline{4} 5}=\left(1-x_{2}\right) x_{3}\left(1-x_{4}\right) x_{5} \text {, } \\
& \lambda_{12 \overline{3} \overline{4} 5}=0 \text {, } \\
& \lambda_{\overline{1} 2 \overline{3} \overline{4} 5}=x_{2}\left(1-x_{3}\right)\left(1-x_{4}\right) x_{5}, \\
& \lambda_{1 \overline{2} \overline{3} \overline{4} 5}=0 \text {, } \\
& \lambda_{1234 \overline{5}}=0, \\
& \lambda_{\overline{1} 234 \overline{5}}=x_{2} x_{3} x_{4}\left(1-x_{5}\right), \\
& \lambda_{12} 34 \overline{5}=0 \text {, } \\
& \lambda_{\overline{1} \overline{2} 34 \overline{5}}=\left(1-x_{2}\right) x_{3} x_{4}\left(1-x_{5}\right) \text {, } \\
& \lambda_{12 \overline{3} 4 \overline{5}}=0 \\
& \lambda_{\overline{1} 2 \overline{3} 4 \overline{5}}=x_{2}\left(1-x_{3}\right) x_{4}\left(1-x_{5}\right) \text {, } \\
& \lambda_{1 \overline{2} \overline{3} 4 \overline{5}}=0 \\
& \lambda_{\overline{1} \overline{2} \overline{3} 4 \overline{5}}=\left(1-x_{2}\right)\left(1-x_{3}\right) x_{4}\left(1-x_{5}\right) \text {, } \\
& \lambda_{123 \overline{4} \overline{5}}=0 \text {, } \\
& \lambda_{\overline{1} 23 \overline{4} \overline{5}}=x_{2} x_{3}\left(1-x_{4}\right)\left(1-x_{5}\right) \text {, } \\
& \lambda_{1 \overline{2} 3 \overline{4} \overline{5}}=0 \text {, } \\
& \lambda_{\overline{1} \overline{2} 3 \overline{4} \overline{5}}=\left(1-x_{2}\right) x_{3}\left(1-x_{4}\right)\left(1-x_{5}\right) \text {, } \\
& \lambda_{12 \overline{3} \overline{4} \overline{5}}=0 \text {, } \\
& \lambda_{\overline{1} 2 \overline{3} \overline{4} \overline{5}}=x_{2}\left(1-x_{3}\right)\left(1-x_{4}\right)\left(1-x_{5}\right) \text {, } \\
& \lambda_{1 \overline{2} \overline{3} \overline{5}}=0 \text {, } \\
& \lambda_{\overline{1} \overline{2} \overline{3} \overline{4} 5}=\left(1-x_{2}\right)\left(1-x_{3}\right)\left(1-x_{4}\right) x_{5}, \quad \lambda_{\overline{1} \overline{2} \overline{3} \overline{5}}=\left(1-x_{2}\right)\left(1-x_{3}\right)\left(1-x_{4}\right)\left(1-x_{5}\right) .
\end{aligned}
$$

(e) $0<T_{L}\left(x_{1}, \ldots, x_{h}\right)<1, T_{L}\left(x_{1}, \ldots, x_{h+1}\right)=\cdots=T_{L}\left(x_{1}, \ldots, x_{5}\right)=0$, with $1 \leqslant h<4$; the solution is given in (56). If for instance $h=3$, the components of the vector $\Lambda_{5}$ are

$$
\begin{aligned}
& \lambda_{12345}=0, \\
& \lambda_{\overline{1} 2345}=\left(1-x_{1}\right) \frac{x_{4}}{3-x_{1}-x_{2}-x_{3}} x_{5}, \\
& \lambda_{1234 \overline{5}}=0, \\
& \lambda_{12} 345=\left(1-x_{2}\right) \frac{x_{4}}{3-x_{1}-x_{2}-x_{3}} x_{5} \text {, } \\
& \lambda_{\overline{1} 234 \overline{5}}=\left(1-x_{1}\right) \frac{x_{4}}{3-x_{1}-x_{2}-x_{3}}\left(1-x_{5}\right) \text {, } \\
& \lambda_{\overline{1} \overline{2} 345}=0 \text {, } \\
& \lambda_{1 \overline{2} 34 \overline{5}}=\left(1-x_{2}\right) \frac{x_{4}}{3-x_{1}-x_{2}-x_{3}}\left(1-x_{5}\right) \text {, } \\
& \lambda_{\overline{1} \overline{2} 345}=0 \text {, } \\
& \lambda_{12 \overline{3} 45}=\left(1-x_{3}\right) \frac{x_{4}}{3-x_{1}-x_{2}-x_{3}} x_{5}, \\
& \lambda_{12 \overline{3} 4 \overline{5}}=\left(1-x_{3}\right) \frac{x_{4}}{3-x_{1}-x_{2}-x_{3}}\left(1-x_{5}\right) \text {, } \\
& \lambda_{\overline{1} 2 \overline{3} 45}=0 \text {, } \\
& \lambda_{1 \overline{2} \overline{3} 45}=0 \text {, } \\
& \lambda_{\overline{1} \overline{2} \overline{3} 45}=0 \text {, } \\
& \lambda_{\overline{1} 2 \overline{3} 4 \overline{5}}=0 \text {, } \\
& \lambda_{1 \overline{2} \overline{3} 4 \overline{5}}=0 \text {, } \\
& \lambda_{\overline{1} \overline{2} \overline{3} 4 \overline{5}}=0, \\
& \lambda_{123 \overline{4} 5}=\left(x_{1}+x_{2}+x_{3}-2\right) x_{5}, \\
& \lambda_{123 \overline{4} \overline{5}}=\left(x_{1}+x_{2}+x_{3}-2\right)\left(1-x_{5}\right), \\
& \lambda_{\overline{1} 23 \overline{4} 5}=\left(1-x_{1}\right)\left(1-\frac{x_{4}}{3-x_{1}-x_{2}-x_{3}}\right) x_{5}, \quad \lambda_{\overline{1} 23 \overline{4} \overline{5}}=\left(1-x_{1}\right)\left(1-\frac{x_{4}}{3-x_{1}-x_{2}-x_{3}}\right)\left(1-x_{5}\right) \text {, } \\
& \lambda_{1 \overline{2} 3 \overline{4} 5}=\left(1-x_{2}\right)\left(1-\frac{x_{4}}{3-x_{1}-x_{2}-x_{3}}\right) x_{5}, \quad \lambda_{1 \overline{2} 3 \overline{4} \overline{5}}=\left(1-x_{2}\right)\left(1-\frac{x_{4}}{3-x_{1}-x_{2}-x_{3}}\right)\left(1-x_{5}\right) \text {, } \\
& \lambda_{\overline{1} \overline{2} 3 \overline{4} 5}=0 \text {, } \\
& \lambda_{\overline{1} \overline{2} 3 \overline{4} \overline{5}}=0 \\
& \lambda_{12 \overline{3} \overline{4} 5}=\left(1-x_{3}\right)\left(1-\frac{x_{4}}{3-x_{1}-x_{2}-x_{3}}\right) x_{5} \text {, } \\
& \lambda_{\overline{1} 2 \overline{3} \overline{4} 5}=0 \text {, } \\
& \lambda_{1 \overline{2} \overline{3} \overline{4} 5}=0 \text {, } \\
& \lambda_{\overline{1} \overline{2} \overline{3} \overline{4} 5}=0 \text {, } \\
& \lambda_{12 \overline{3} \overline{4} \overline{5}}=\left(1-x_{3}\right)\left(1-\frac{x_{4}}{3-x_{1}-x_{2}-x_{3}}\right)\left(1-x_{5}\right) \text {, } \\
& \lambda_{\overline{1} 2 \overline{3} \overline{4} \overline{5}}=0 \text {, } \\
& \lambda_{1 \overline{2} \overline{3} \overline{5} \overline{5}}=0 \text {, } \\
& \lambda_{\overline{1} \overline{2} \overline{3} \overline{4} \overline{5}}=0 .
\end{aligned}
$$


(f) $T_{L}\left(x_{1}, \ldots, x_{h}\right)=1, T_{L}\left(x_{1}, \ldots, x_{h+1}\right)=\cdots=T_{L}\left(x_{1}, \ldots, x_{5}\right)=0$, with $1 \leqslant h<4$; the solution is given in (58). If for instance $h=2$, the components of the vector $\Lambda_{5}$ are

$$
\left\{\begin{array}{l}
\lambda_{12 \overline{3} 45}=x_{4} x_{5}, \quad \lambda_{12 \overline{3} 4 \overline{5}}=x_{4}\left(1-x_{5}\right), \quad \lambda_{12 \overline{3} \overline{4} 5}=\left(1-x_{4}\right) x_{5} \\
\lambda_{12 \overline{3} \overline{4} \overline{5}}=\left(1-x_{4}\right)\left(1-x_{5}\right), \quad \lambda_{1 * 2 * 3 * 4 * 5 *}=0, \text { otherwise }
\end{array}\right.
$$

\section{Probabilistic interpretation of Frank t-norms and t-conorms}

In this section we show that the previsions of the conjunction and the disjunction of $n$ conditional events can be represented as a Frank t-norm $T_{\lambda}$ and a Frank t-conorm $S_{\lambda}$, respectively. Then, we characterize the set of coherent assessments by Frank t-norms and t-conorms. Moreover, when $n=2$, we show that, under logical independence, $T_{\lambda}(A|H, B| K)=(A \mid H) \wedge(B \mid K)$ and $S_{\lambda}(A|H, B| K)=(A \mid H) \vee(B \mid K)$ for every $\lambda \in[0,+\infty]$. We also examine cases where there are logical dependencies.

\subsection{Set of coherent assessments, Frank t-norms and t-conorms}

We recall that the $n$-ary Frank t-norm, with parameter $\lambda \in[0,+\infty]$, is

$$
T_{\lambda}\left(x_{1}, \ldots, x_{n}\right)= \begin{cases}T_{M}\left(x_{1}, \ldots, x_{n}\right)=\min \left\{x_{1}, \ldots, x_{n}\right\}, & \text { if } \lambda=0, \\ T_{P}\left(x_{1}, \ldots, x_{n}\right)=\prod_{i=1}^{n} x_{i}, & \text { if } \lambda=1, \\ T_{L}\left(x_{1}, \ldots, x_{n}\right)=\max \left\{\sum_{i=1}^{n} x_{i}-n+1,0\right\}, & \text { if } \lambda=+\infty, \\ \log _{\lambda}\left(1+\frac{\prod_{i=1}^{n}\left(\lambda^{x_{i}}-1\right)}{(\lambda-1)^{n-1}}\right), & \text { otherwise. }\end{cases}
$$

The next result shows that, under logical independence, given any coherent assessment $\left(x_{1}, \ldots, x_{n}, x_{1 \cdots n}\right)$ on $\left\{E_{1}\left|H_{1}, \ldots, E_{n}\right| H_{n}, \mathcal{C}_{1 \cdots n}\right\}$, it holds that $x_{1 \cdots n}=T_{\lambda}\left(x_{1}, \cdots, x_{n}\right)$ for some $\lambda \in[0,+\infty]$; conversely, for every $\lambda \in[0,+\infty]$ the extension $x_{1 \cdots n}=T_{\lambda}\left(x_{1}, \cdots, x_{n}\right)$ is coherent.

Theorem 12. Let $E_{1}, \ldots, E_{n}, H_{1}, \ldots, H_{n}$ be logically independents events, with $H_{1} \neq \varnothing, \ldots, H_{n} \neq \varnothing$, $n \geqslant 2$. The set $\Pi$ of all prevision coherent assessments $\mathcal{M}=\left(x_{1}, \ldots, x_{n}, x_{1 \ldots n}\right)$ on the family $\mathcal{F}=$ $\left\{E_{1}\left|H_{1}, \ldots, E_{n}\right| H_{n}, \mathcal{C}_{1 \cdots n}\right\}$ coincides with the set

$$
\Pi_{T}=\left\{\left(x_{1}, \ldots, x_{n}, x_{1 \cdots n}\right):\left(x_{1}, \ldots, x_{n}\right) \in[0,1]^{n}, x_{1 \cdots n}=T_{\lambda}\left(x_{1}, \cdots, x_{n}\right), \lambda \in[0,+\infty]\right\} .
$$

Proof. We show that $\Pi \subseteq \Pi_{T}$ and $\Pi_{T} \subseteq \Pi$. For each given $\mathcal{M}=\left(x_{1}, \ldots, x_{n}, x_{1 \ldots n}\right) \in \Pi$, by Theorem [10, it holds that $\left(x_{1}, \ldots, x_{n}\right) \in[0,1]^{n}$ and $x_{1 \ldots n} \in\left[T_{L}\left(x_{1}, \ldots, x_{n}\right), T_{M}\left(x_{1}, \ldots, x_{n}\right)\right]=$ $\left[T_{+\infty}\left(x_{1}, \ldots, x_{n}\right), T_{0}\left(x_{1}, \ldots, x_{n}\right)\right]$. Then, by the continuity property of $T_{\lambda}$ with respect to $\lambda$, there exists $\lambda \in[0,+\infty]$ such that $x_{1 \cdots n}=T_{\lambda}\left(x_{1}, \ldots, x_{n}\right)$. Thus, $\Pi \subseteq \Pi_{T}$.

Conversely, for every $\lambda \in[0,+\infty]$ and for every $\left(x_{1}, \ldots, x_{n}\right) \in[0,1]^{n}$, by Theorem 10 the assessment $\mathcal{M}=\left(x_{1}, \ldots, x_{n}, T_{\lambda}\left(x_{1}, \ldots, x_{n}\right)\right)$ is coherent because $T_{\lambda}\left(x_{1}, \ldots, x_{n}\right) \in\left[T_{L}\left(x_{1}, \ldots, x_{n}\right), T_{M}\left(x_{1}, \ldots, x_{n}\right)\right]$. Thus $\Pi_{T} \subseteq \Pi$ and hence $\Pi=\Pi_{T}$.

Remark 5. We observe that in case of some logical dependencies, for each given coherent assessment $\left(x_{1}, \ldots, x_{n}\right)$, the set of coherent extensions $x_{1 \cdots n}$ is an interval

$$
\left[\mu^{\prime}\left(x_{1}, \ldots, x_{n}\right), \mu^{\prime \prime}\left(x_{1} \cdots x_{n}\right)\right] \subseteq\left[T_{+\infty}\left(x_{1}, \ldots, x_{n}\right), T_{0}\left(x_{1}, \ldots, x_{n}\right)\right] .
$$

By Theorem 12, there exist $\lambda^{\prime}$ and $\lambda^{\prime \prime}$ such that $\mu^{\prime}\left(x_{1}, \ldots, x_{n}\right)=T_{\lambda^{\prime}}\left(x_{1}, \ldots, x_{n}\right)$ and $\mu^{\prime \prime}\left(x_{1}, \ldots, x_{n}\right)=$ $T_{\lambda^{\prime \prime}}\left(x_{1}, \ldots, x_{n}\right)$, with $+\infty \geqslant \lambda^{\prime} \geqslant \lambda^{\prime \prime} \geqslant 0$ because $T_{\lambda}$ is decreasing with respect to the parameter $\lambda$. Then,

$$
\left[\mu^{\prime}\left(x_{1}, \ldots, x_{n}\right), \mu^{\prime \prime}\left(x_{1} \cdots x_{n}\right)\right]=\left[T_{\lambda^{\prime}}\left(x_{1}, \ldots, x_{n}\right), T_{\lambda^{\prime \prime}}\left(x_{1}, \ldots, x_{n}\right)\right] .
$$


Moreover, for each $x_{1 \cdots n} \in\left[\mu^{\prime}\left(x_{1}, \ldots, x_{n}\right), \mu^{\prime \prime}\left(x_{1} \cdots x_{n}\right)\right]$, there exists $\lambda \in\left[\lambda^{\prime \prime}, \lambda^{\prime}\right]$ such that $x_{1 \cdots n}=$ $T_{\lambda}\left(x_{1}, \ldots, x_{n}\right)$. However, the set of all coherent assessments $\left(x_{1}, \ldots, x_{n}, x_{1 \cdots n}\right)$ is in general a subset of the set $\Pi_{T}$ given in (60). Then, it may happen that, given any coherent assessment $\left(x_{1}, \ldots, x_{n}\right)$, the extension $x_{1 \cdots n}=T_{\lambda}\left(x_{1}, \ldots, x_{n}\right)$ is not coherent, for some $\lambda \in[0,+\infty]$.

We now show that a result dual of Theorem (12) holds for the disjunction of conditional events, where the Frank t-norm is replaced by the dual Frank t-conorm. The notion of disjunction given in Definition 3 can be extended to the case of $n$ conditional events $E_{1}\left|H_{1}, \ldots, E_{n}\right| H_{n}$ ([33]). Moreover, the conjunction $\mathcal{C}_{1 \cdots n}$ and the disjunction $\mathcal{D}_{1 \ldots n}$ satisfy De Morgan's Laws; in particular

$$
\mathcal{D}_{1 \cdots n}=\bigvee_{i=1}^{n}\left(E_{i} \mid H_{i}\right)=1-\bigwedge_{i=1}^{n}\left(\bar{E}_{i} \mid H_{i}\right)=1-\mathcal{C}_{\overline{1} \cdots \bar{n}},
$$

where $\mathcal{C}_{\overline{1} \cdots \bar{n}}=\bigwedge_{i=1}^{n}\left(\bar{E}_{i} \mid H_{i}\right)$. We set $\mathbb{P}\left(\mathcal{D}_{1 \cdots n}\right)=y_{1 \cdots n}$ and $\mathbb{P}\left(\mathcal{C}_{\overline{1} \cdots \bar{n}}\right)=x_{\overline{1} \ldots \bar{n}}$. Of course, $y_{1 \cdots n}=1-x_{\overline{1} \cdots \bar{n}}$. By Theorem 10, $x_{1 \cdots \bar{n}}$ is a coherent extension of the assessment $\left(x_{1}, \ldots, x_{n}\right)$ on $\left\{E_{1}\left|H_{1}, \ldots, E_{n}\right| H_{n}\right\}$ if and only if

$$
T_{L}\left(1-x_{1}, \ldots, 1-x_{n}\right) \leqslant x_{1} \ldots \bar{n} \leqslant T_{M}\left(1-x_{1}, \ldots, 1-x_{n}\right) .
$$

that is

$$
1-T_{M}\left(1-x_{1}, \ldots, 1-x_{n}\right) \leqslant y_{1 \cdots n} \leqslant 1-T_{L}\left(1-x_{1}, \ldots, 1-x_{n}\right) .
$$

Moreover, denoting by $S_{L}$ and $S_{M}$ the Lukasiewicz and Minimum t-conorms, respectively, it holds that

$$
S_{L}\left(x_{1}, \ldots, x_{n}\right)=\min \left\{\sum_{i=1}^{n} x_{i}, 1\right\}=1-T_{L}\left(1-x_{1}, \ldots, 1-x_{n}\right),
$$

and

$$
S_{M}\left(x_{1}, \ldots, x_{n}\right)=\max \left\{x_{1}, \ldots, x_{n}\right\}=1-T_{M}\left(1-x_{1}, \ldots, 1-x_{n}\right) .
$$

Then, from (61), we obtain the result below which establishes the Fréchet-Hoeffding bounds for the disjunction of $\mathrm{n}$ conditional events.

Theorem 13. Let $E_{1}, \ldots, E_{n}, H_{1}, \ldots, H_{n}$ be logically independents events, with $H_{1} \neq \varnothing, \ldots, H_{n} \neq \varnothing, n \geqslant$ 2. The set of all prevision coherent assessments $\left(x_{1}, \ldots, x_{n}, y_{1 \ldots n}\right)$ on the family $\left\{E_{1}\left|H_{1}, \ldots, E_{n}\right| H_{n}, \mathcal{D}_{1 \ldots n}\right\}$ is the set

$$
\Gamma=\left\{\left(x_{1}, \ldots, x_{n}, y_{1 \cdots n}\right):\left(x_{1}, \ldots, x_{n}\right) \in[0,1]^{n}, y_{1 \cdots n} \in\left[S_{M}\left(x_{1}, \ldots, x_{n}\right), S_{L}\left(x_{1}, \ldots, x_{n}\right)\right]\right\} .
$$

Based on Theorems 12 and 13 , we have

Theorem 14. Let $E_{1}, \ldots, E_{n}, H_{1}, \ldots, H_{n}$ be logically independents events, with $H_{1} \neq \varnothing, \ldots, H_{n} \neq \varnothing$, $n \geqslant 2$. The set $\Gamma$ of all prevision coherent assessments $\mathcal{M}=\left(x_{1}, \ldots, x_{n}, y_{1 \ldots n}\right)$ on the family $\mathcal{F}=$ $\left\{E_{1}\left|H_{1}, \ldots, E_{n}\right| H_{n}, \mathcal{D}_{1 \ldots n}\right\}$ coincides with the set

$$
\Gamma_{S}=\left\{\left(x_{1}, \ldots, x_{n}, y_{1 \cdots n}\right):\left(x_{1}, \ldots, x_{n}\right) \in[0,1]^{n}, y_{1 \cdots n}=S_{\lambda}\left(x_{1}, \cdots, x_{n}\right), \lambda \in[0,+\infty]\right\} .
$$




\subsection{Representation of conjunction and disjunction of two conditional events}

In this section we examine the representation of conjunction and disjunction of two conditional events in terms of Frank t-norms and Frank t-conorms, respectively.

Theorem 15. For each coherent prevision assessment $(x, y, z)$ on $\{A|H, B| K,(A \mid H) \wedge(B \mid K)\}$, it holds that

$$
(A \mid H) \wedge(B \mid K)=T_{\lambda}(A|H, B| K), \text { for some } \lambda \in[0,+\infty] .
$$

Proof. From Definition 5 it holds that $T_{\lambda}(1,1)=1, T_{\lambda}(x, 0)=T_{\lambda}(0, y)=0, T_{\lambda}(x, 1)=x, T_{\lambda}(1, y)=y$. Then,

$$
T_{\lambda}(A|H, B| K)= \begin{cases}1, & \text { if } A H B K \text { is true, } \\ 0, & \text { if } \bar{A} H \text { is true or } \bar{B} K \text { is true } \\ x, & \text { if } \bar{H} B K \text { is true, } \\ y, & \text { if } \bar{K} A H \text { is true, } \\ T_{\lambda}(x, y), & \text { if } \bar{H} \bar{K} \text { is true. }\end{cases}
$$

By Remark [5] there exists $\lambda \in[0,+\infty]$ such that $z=T_{\lambda}(x, y)$. Then, from (9) and (65), for each coherent assessment $(x, y, z)$ on $\{A|H, B| K,(A \mid H) \wedge(B \mid K)\}$ there exists $\lambda \in[0,+\infty]$ such that $(A \mid H) \wedge(B \mid K)=$ $T_{\lambda}(A|H, B| K)$.

Remark 6. We observe that to define the conjunction $(A \mid H) \wedge(B \mid K)$ amounts to specify a coherent assessment $(x, y, z)$ on $\{A|H, B| K,(A \mid H) \wedge(B \mid K)\}$. Moreover, we recall that, by Theorem 12] (see also formula (12) $)$, in the particular case of logical independence of $A, B, H, K$, for each $\lambda \in[0,+\infty]$ the extension $z=T_{\lambda}(x, y)$ on $(A \mid H) \wedge(B \mid K)$ of the assessment $(x, y)$ on $\{A|H, B| K\}$ is coherent, for every $(x, y) \in[0,1]^{2}$. Then, for any given assessment $\left(x, y, T_{\lambda}(x, y)\right)$ on $\{A|H, B| K,(A \mid H) \wedge(B \mid K)\}$, with $(x, y) \in[0,1]^{2}, \lambda \in[0,+\infty]$ it holds that

$$
(A \mid H) \wedge(B \mid K)=T_{\lambda}(A|H, B| K) .
$$

In other words, for every $\lambda \in[0,+\infty]$, it is possible to define the conjunction as $(A \mid H) \wedge(B \mid K)=$ $T_{\lambda}(A|H, B| K)$, for every $(x, y) \in[0,1]^{2}$. Of course, in case of some logical dependencies, given a coherent assessment $(x, y)$, it may happen that $T_{\lambda}(A|H, B| K)$ is not a conjunction for some $\lambda \in[0,+\infty]$ because, by Remark [5, the extension $z=T_{\lambda}(x, y)$ is not coherent. In Section 5 we will give an example where $T_{\lambda}(A|H, A| K)$, with $\lambda>1$, does not represent the conjunction $(A \mid H) \wedge(A \mid K)$ for some coherent $(x, y)$.

We recall that the dual Frank t-conorm $S_{\lambda}(x, y)=1-T_{\lambda}(1-x, 1-y)$ is defined as

$$
S_{\lambda}(x, y)= \begin{cases}S_{M}(x, y)=\max \{x, y\}, & \text { if } \lambda=0, \\ S_{P}(x, y)=x+y-x y, & \text { if } \lambda=1, \\ S_{L}(x, y)=\min \{x+y, 1\}, & \text { if } \lambda=+\infty, \\ 1-\log _{\lambda}\left(1+\frac{\left(\lambda^{1-x}-1\right)\left(\lambda^{1-y}-1\right)}{\lambda-1}\right), & \text { otherwise. }\end{cases}
$$

Moreover, for every $\lambda \in[0,+\infty]$, the pair $\left(T_{\lambda}, S_{\lambda}\right)$ satisfies the functional equation ([41, Theorem 5.14])

$$
S_{\lambda}(x, y)=x+y-T_{\lambda}(x, y), \quad(x, y) \in[0,1]^{2} .
$$

Theorem 16. For each coherent prevision assessment $(x, y, w)$ on $\{A|H, B| K,(A \mid H) \vee(B \mid K)\}$, it holds that

$$
(A \mid H) \vee(B \mid K)=S_{\lambda}(A|H, B| K) \text {, for some } \lambda \in[0,+\infty] .
$$


Proof. From (66) it holds that $S_{\lambda}(1,1)=1, S_{\lambda}(x, 1)=S_{\lambda}(1, y)=1, S_{\lambda}(x, 0)=x, S_{\lambda}(0, y)=y$. Then,

$$
S_{\lambda}(A|H, B| K)= \begin{cases}1, & \text { if } A H \vee B K \text { is true, } \\ 0, & \text { if } \bar{A} H \bar{B} H \text { is true, } \\ x, & \text { if } \bar{H} \bar{B} K \text { is true, } \\ y, & \text { if } \bar{K} \bar{A} H \text { is true, } \\ S_{\lambda}(x, y), & \text { if } \bar{H} \bar{K} \text { is true. }\end{cases}
$$

By recalling the prevision sum rule ([30, Section 6]), it holds that

$$
\mathbb{P}[(A \mid H) \vee(B \mid K)]=P(A \mid H)+P(B \mid K)-\mathbb{P}[(A \mid H) \wedge(B \mid K)],
$$

that is $w=x+y-z$, where $z=\mathbb{P}[(A \mid H) \wedge(B \mid K)]$. Moreover, by Theorem[15there exists $\lambda \in[0,+\infty]$ such that $z=T_{\lambda}(x, y)$. Then, $w=x+y-T_{\lambda}(x, y)$ and hence, from [67), there exists $\lambda \in[0,+\infty]$ such that $w=$ $S_{\lambda}(x, y)$. Finally, from (13) and (69), for each coherent assessment $(x, y, z)$ on $\{A|H, B| K,(A \mid H) \vee(B \mid K)\}$ there exists $\lambda \in[0,+\infty]$ such that $(A \mid H) \vee(B \mid K)=S_{\lambda}(A|H, B| K)$.

As a further comment, we also observe that, for each coherent assessment $(x, y, z, w)$ on the family $\{A|H, B| K,(A \mid H) \wedge(B \mid K),(A \mid H) \vee(B \mid K)\}$, there exists $\lambda \in[0,+\infty]$ such that

$$
(A \mid H) \vee(B \mid K)=(A \mid H)+(B \mid K)-T_{\lambda}(A|H, B| K)=S_{\lambda}(A|H, B| K) .
$$

We remark that in the case of some logical dependencies among the basic events $A, B, H, K$, the Frank t-norm may represent the conjunction only for the values of $\lambda$ in a subset of $[0,+\infty]$. In the next section we examine a case where the subset is the interval $[0,1]$.

\subsection{The conjunction $(A \mid H) \wedge(B \mid K)$, when $A=B$}

In this section we examine a case of logical dependencies by considering the conjunction $(A \mid H) \wedge(B \mid K)$ when $A=B$, that is $(A \mid H) \wedge(A \mid K)$. By setting $P(A \mid H)=x, P(A \mid K)=y$ and $\mathbb{P}[(A \mid H) \wedge(A \mid K)]=z$, it holds that

$$
(A \mid H) \wedge(A \mid K)= \begin{cases}1, & \text { if } A H K \text { is true, } \\ 0, & \text { if } \bar{A} H \vee \bar{A} K \text { is true, } \\ x, & \text { if } \bar{H} A K \text { is true, } \\ y, & \text { if } A H \bar{K} \text { is true, } \\ z, & \text { if } \bar{H} \bar{K} \text { is true }\end{cases}
$$

that is

$$
(A \mid H) \wedge(A \mid K)=A H K+x \bar{H} A K+y \bar{K} A H+z \bar{H} \bar{K} .
$$

In the next result we show that, for each coherent assessment $(x, y)$, the lower bound on $z$ is, not $T_{L}(x, y)$, but $T_{P}(x, y)$; the upper bound is still $T_{M}(x, y)$.

Theorem 17. Let $A, H, K$ be three logically independent events, with $H \neq \varnothing, K \neq \varnothing$. The set $\Pi$ of all coherent assessments $(x, y, z)$ on the family $\mathcal{F}=\{A|H, A| K,(A \mid H) \wedge(A \mid K)\}$ is given by

$$
\Pi=\left\{(x, y, z):(x, y) \in[0,1]^{2}, T_{P}(x, y)=x y \leqslant z \leqslant \min \{x, y\}=T_{M}(x, y)\right\} .
$$


Proof. We recall that, by Example 1, the assessment $(x, y)$ is coherent for every $(x, y) \in[0,1]^{2}$. Given any coherent assessment $(x, y)$, by Theorem 6 there exists an interval $\left[z^{\prime}, z^{\prime \prime}\right]$ of coherent extensions $z$ to $(A \mid H) \wedge(A \mid K)$. We will show that $z^{\prime}=x y$ and $z^{\prime \prime}=\min \{x, y\}$. Let $\mathcal{M}=(x, y, z)$ be a prevision assessment on $\mathcal{F}$, with $(x, y) \in[0,1]^{2}$. The constituents associated with the pair $(\mathcal{F}, \mathcal{M})$ and contained in $H \vee K$ are: $C_{1}=A H K, C_{2}=\bar{A} H K, C_{3}=\bar{A} \bar{H} K, C_{4}=\bar{A} H \bar{K}, C_{5}=A \bar{H} K, C_{6}=A H \bar{K}$. The associated points $Q_{h}$ 's are $Q_{1}=(1,1,1), Q_{2}=(0,0,0), Q_{3}=(x, 0,0), Q_{4}=(0, y, 0), Q_{5}=(x, 1, x), Q_{6}=(1, y, y)$. With the further constituent $C_{0}=\bar{H} \bar{K}$ it is associated the point $Q_{0}=\mathcal{M}=(x, y, z)$. Considering the convex hull $\mathcal{I}$ (see Figure 110 of $Q_{1}, \ldots, Q_{6}$, a necessary condition for the coherence of the prevision assessment $\mathcal{M}=(x, y, z)$ on $\mathcal{F}$ is that $\mathcal{M} \in \mathcal{I}$, that is the following system must be solvable

$$
(\Sigma)\left\{\begin{array}{l}
\lambda_{1}+x \lambda_{3}+x \lambda_{5}+\lambda_{6}=x, \quad \lambda_{1}+y \lambda_{4}+\lambda_{5}+y \lambda_{6}=y, \quad \lambda_{1}+x \lambda_{5}+y \lambda_{6}=z \\
\sum_{h=1}^{6} \lambda_{h}=1, \quad \lambda_{h} \geqslant 0, h=1, \ldots, 6
\end{array}\right.
$$

First of all, we observe that solvability of $(\Sigma)$ requires that $z \leqslant x$ and $z \leqslant y$, that is $z \leqslant \min \{x, y\}$; thus $z^{\prime \prime} \leqslant \min \{x, y\}$. We now verify that $(x, y, z)$, with $(x, y) \in[0,1]^{2}$ and $z=\min \{x, y\}$, is coherent, from which it follows that $z^{\prime \prime}=\min \{x, y\}$. We distinguish two cases: (i) $x \leqslant y$ and (ii) $x>y$.

Case $(i)$. In this case $z=\min \{x, y\}=x$. If $y=0$ the system $(\Sigma)$ becomes

$$
\lambda_{1}+\lambda_{6}=0, \quad \lambda_{1}+\lambda_{5}=0, \quad \lambda_{1}=0, \lambda_{2}+\lambda_{3}+\lambda_{4}=1, \quad \lambda_{h} \geqslant 0, \quad h=1, \ldots, 6 .
$$

which is clearly solvable. In particular there exist solutions with $\lambda_{2}>0, \lambda_{3}>0, \lambda_{4}>0$, by Theorem 5 , as the set $I_{0}$ is empty the solvability of $(\Sigma)$ is sufficient for coherence of the assessment $(0,0,0)$. If $y>0$ the system $(\Sigma)$ is solvable and a solution is $\Lambda=\left(\lambda_{1}, \ldots, \lambda_{6}\right)=\left(x, \frac{x(1-y)}{y}, 0, \frac{y-x}{y}, 0,0\right)$. We observe that, if $x>0$, then $\lambda_{1}>0$ and $I_{0}=\varnothing$ because $\mathcal{C}_{1}=H K \subseteq H \vee K$, so that $\mathcal{M}=(x, y, x)$ is coherent. If $x=0$ (and hence $z=0$ ), then $\lambda_{4}=1$ and $I_{0} \subseteq\{2\}$. Then, as the sub-assessment $P(A \mid K)=y$ is coherent, it follows that the assessment $\mathcal{M}=(0, y, 0)$ is coherent too.

Case $(i i)$. The system is solvable and a solution is $\Lambda=\left(\lambda_{1}, \ldots, \lambda_{6}\right)=\left(y, \frac{y(1-x)}{x}, \frac{x-y}{x}, 0,0,0\right)$. We observe that, if $y>0$, then $\lambda_{1}>0$ and $I_{0}=\varnothing$ because $\mathcal{C}_{1}=H K \subseteq H \vee K$, so that $\mathcal{M}=(x, y, y)$ is coherent. If $y=0$ (and hence $z=0$ ), then $\lambda_{3}=1$ and $I_{0} \subseteq\{1\}$. Then, as the sub-assessment $P(A \mid H)=x$ is coherent, it follows that the assessment $\mathcal{M}=(x, 0,0)$ is coherent too. Thus, for every $(x, y) \in[0,1]^{2}$, the assessment $(x, y, \min \{x, y\})$ is coherent and hence the upper bound on $z$ is $z^{\prime \prime}=\min \{x, y\}=T_{M}(x, y)$.

We now verify that $(x, y, x y)$, with $(x, y) \in[0,1]^{2}$ is coherent; moreover we show that $(x, y, z)$, with $z<x y$, is not coherent and the lower bound for $z$ is $z^{\prime}=x y$. First of all, we observe that $\mathcal{M}=(1-x) Q_{4}+x Q_{6}$, so that a solution of $(\Sigma)$ is $\Lambda_{1}=(0,0,0,1-x, 0, x)$. Moreover, $\mathcal{M}=(1-y) Q_{3}+y Q_{5}$, so that another solution is $\Lambda_{2}=(0,0,1-y, 0, y, 0)$. Then $\Lambda=\frac{\Lambda_{1}+\Lambda_{2}}{2}=\left(0,0, \frac{1-y}{2}, \frac{1-x}{2}, \frac{y}{2}, \frac{x}{2}\right)$ is a solution of $(\Sigma)$ such that $I_{0}=\varnothing$. Thus the assessment $(x, y, x y)$ is coherent for every $(x, y) \in[0,1]^{2}$. In order to verify that $x y$ is the lower bound on $z$ we observe that the points $Q_{3}, Q_{4}, Q_{5}, Q_{6}$ belong to a plane $\pi$ of equation: $y X+x Y-Z=x y$, where $X, Y, Z$ are the axis' coordinates. Now, by considering the function $f(X, Y, Z)=y X+x Y-Z$, we observe that for each constant $k$ the equation $f(X, Y, Z)=k$ represents a plane which is parallel to $\pi$ and coincides with $\pi$ when $k=x y$. We also observe that $f\left(Q_{1}\right)=f(1,1,1)=x+y-1=T_{L}(x, y) \leqslant$ $x y=T_{P}(x, y), f\left(Q_{2}\right)=f(0,0,0)=0 \leqslant x y=T_{P}(x, y)$, and $f\left(Q_{3}\right)=f\left(Q_{4}\right)=f\left(Q_{5}\right)=f\left(Q_{6}\right)=$ $x y=T_{P}(x, y)$. Then, for every $\mathcal{P}=\sum_{h=1}^{6} \lambda_{h} Q_{h}$, with $\lambda_{h} \geqslant 0$ and $\sum_{h=1}^{6} \lambda_{h}=1$, that is $\mathcal{P} \in \mathcal{I}$, it holds that $f(\mathcal{P})=f\left(\sum_{h=1}^{6} \lambda_{h} Q_{h}\right)=\sum_{h=1}^{6} \lambda_{h} f\left(Q_{h}\right) \leqslant x y$. On the other hand, given any $a>0$, by considering $\mathcal{P}=(x, y, x y-a)$ it holds that $f(\mathcal{P})=f(x, y, x y-a)=x y+x y-x y+a=x y+a>x y$. Therefore, for any given $a>0$ the assessment $(x, y, x y-a)$ is not coherent because $(x, y, x y-a) \notin \mathcal{I}$. Then, the lower bound on $z$ is $z^{\prime}=x y=T_{P}(x, y)$. Thus, the set of all coherent assessments $(x, y, z)$ on $\mathcal{F}$ is the set $\Pi$ in (70). 


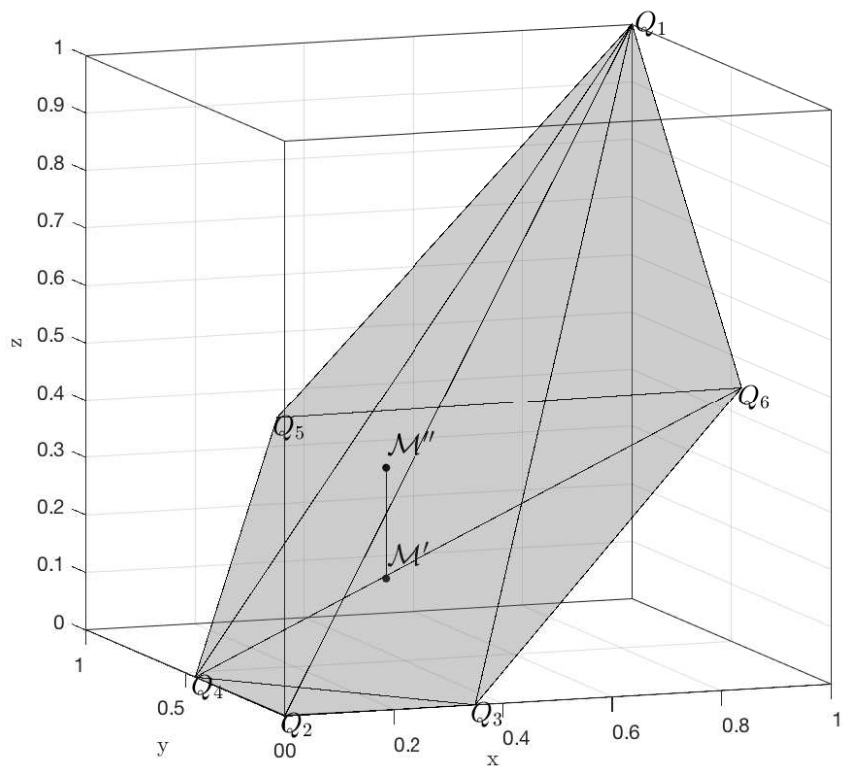

Figure 1: Convex hull $\mathcal{I}$ of the points $Q_{1}, Q_{2}, Q_{3}, Q_{4}, Q_{5}, Q_{6} \cdot \mathcal{M}^{\prime}=\left(x, y, z^{\prime}\right), \mathcal{M}^{\prime \prime}=\left(x, y, z^{\prime \prime}\right)$, where $(x, y) \in[0,1]^{2}, z^{\prime}=x y$, $z^{\prime \prime}=\min \{x, y\}$. In the figure the numerical values are: $x=0.35, y=0.45, z^{\prime}=0.1575$, and $z^{\prime \prime}=0.35$.

Based on Theorem 17, we can give a result which is similar to Theorem 12, with $n=2$; but in this case $\lambda$ belongs to the interval $[0,1]$.

Theorem 18. Let $A, H, K$ be logically independents events, with $H \neq \varnothing, K \neq \varnothing$. The set of all prevision coherent assessments $\mathcal{M}=(x, y, z)$ on the family $\mathcal{F}=\{A|H, A| K,(A \mid H) \wedge(A \mid K)\}$ is the set

$$
\left\{(x, y, z):(x, y) \in[0,1]^{2}, z=T_{\lambda}(x, y), \lambda \in[0,1]\right\} .
$$

Proof. By exploiting Theorem 17 the proof is the same as in Theorem 12

We observe that for every $\lambda \in[0,1]$, it is possible to define the conjunction as $(A \mid H) \wedge(A \mid K)=$ $T_{\lambda}(A|H, A| K)$, for every $(x, y) \in[0,1]^{2}$. Moreover, for some coherent $(x, y)$, it may happen that $T_{\lambda}(A|H, A| K)$ is not a conjunction when $\lambda \in(1+\infty]$, as shown by the example below.

Example 6. Let $A, H, K$ be logically independents events, with $H \neq \varnothing, K \neq \varnothing$. If, for instance, $(x, y)=$ $\left(\frac{1}{2}, \frac{1}{2}\right)$. Then, by Theorem [17, the extension $z$ is coherent if and only if $z \in\left[T_{1}\left(\frac{1}{2}, \frac{1}{2}\right), T_{0}\left(\frac{1}{2}, \frac{1}{2}\right)\right]=\left[\frac{1}{4}, \frac{1}{2}\right]$. Moreover, as $T_{\lambda}\left(\frac{1}{2}, \frac{1}{2}\right)$ is decreasing with respect to $\lambda$, it holds that

$$
T_{\lambda}\left(\frac{1}{2}, \frac{1}{2}\right)<T_{1}\left(\frac{1}{2}, \frac{1}{2}\right)=\frac{1}{4}, \forall \lambda \in(1+\infty] .
$$

Then, the extension $z=T_{\lambda}\left(\frac{1}{2}, \frac{1}{2}\right)$, with $\lambda \in(1+\infty]$, is not coherent; thus, $T_{\lambda}(A|H, A| K)$, with $\lambda \in(1+\infty]$ and $(x, y)=\left(\frac{1}{2}, \frac{1}{2}\right)$, is not a conjunction.

We also observe that, in particular cases, $T_{\lambda}(A|H, A| K)$ is a conjunction when $\lambda \in(1+\infty]$. For instance, if $x=0$, or $y=0$, it holds that, for every $\lambda \in[0,+\infty], z=T_{\lambda}(x, y)=0$ is the unique coherent extension of the assessment $(x, y)$. Then, $T_{\lambda}(A|H, A| K)$, with $x=0$, or $y=0$, is a conjunction for every $\lambda \in[0,+\infty]$.

The next result consider the particular case where $H$ and $K$ are incompatible. 
Theorem 19. Let $A, H, K$ be three events, with $A$ logically independent from both $H$ and $K$, with $H \neq \varnothing$, $K \neq \varnothing, H K=\varnothing$. The set of all coherent assessments $(x, y, z)$ on the family $\mathcal{F}=\{A|H, A| K,(A \mid H) \wedge$ $(A \mid K)\}$ is given by $\left\{(x, y, z):(x, y) \in[0,1]^{2}, z=x y=T_{P}(x, y)\right\}$.

Proof. Let $\mathcal{M}=(x, y, z)$ be a prevision assessment on $\mathcal{F}$. We recall that, by Example 2, the coherence of $(x, y)$ amounts to $(x, y) \in[0,1]^{2}$. Moreover, we observe that $\bar{H} K=K$ and $H \bar{K}=H$ and

$$
(A \mid H) \wedge(A \mid K)=(x A \bar{H} K+y A H \bar{K})|(H \vee K)=x A K|(H \vee K)+y A H \mid(H \vee K) .
$$

Then,

$$
z=x P(A K \mid(H \vee K))+y P(A H \mid(H \vee K))=x y P(K \mid(H \vee K))+x y P(H \mid(H \vee K))=x y=T_{P}(x, y) .
$$

Thus, the set of all coherent assessments $(x, y, z)$ on the family $\mathcal{F}=\{A|H, A| K,(A \mid H) \wedge(A \mid K)\}$ is given by $\left\{(x, y, z):(x, y) \in[0,1]^{2}, z=x y=T_{P}(x, y)\right\}$.

Remark 7. We remark that, when $H K=\varnothing, T_{\lambda}(A|H, A| K)$ represents the conjunction $(A \mid H) \wedge(A \mid K)$ only if $\lambda=1$. Indeed, from Theorems 15 and 19 , it holds that

$$
(A \mid H) \wedge(A \mid K)=(A \mid H) \cdot(A \mid K)=T_{P}(A|H, A| K)=T_{1}(A|H, A| K), \quad \text { when } H K=\varnothing .
$$

We point out again that, as shown by Theorem 18 and by Remark 7 in case of some logical dependencies to assign conditional previsions and to represent conjunctions by means of a Frank t-norm $T_{\lambda}$ is consistent only for some values of $\lambda$. For instance, given any assessment $(x, y)$ on $\{A|H, B| K\}$, with $0<x<1$, $0<y<1$, the assessment $\mathbb{P}[(A \mid H) \wedge(B \mid K)]=T_{L}(x, y)$ is not coherent, because $\max \{x+y-1,0\}<x y$. Moreover, $T_{L}(A|H, A| K)=T_{+\infty}(A|H, A| K)$ is not a conjunction.

\section{Some further results on Frank t-norms}

In this section we give some particular results on Frank t-norms and coherence of prevision assessments on the family $\mathcal{F}=\left\{\mathfrak{C}_{1}, \mathcal{C}_{2}, \mathcal{C}_{3}, \mathcal{C}_{12}, \mathcal{C}_{13}, \mathcal{C}_{23}, \mathcal{C}_{123}\right\}$, where $\mathcal{C}_{i}=E_{i} \mid H_{i}, \mathcal{C}_{i j}=\left(E_{i} \mid H_{i}\right) \wedge\left(E_{j} \mid H_{j}\right)$, and $\mathfrak{C}_{123}=$ $\left(E_{1} \mid H_{1}\right) \wedge\left(E_{2} \mid H_{2}\right) \wedge\left(E_{3} \mid H_{3}\right)$. We set $\mathbb{P}\left(\mathcal{C}_{i}\right)=x_{i}, i=1,2,3, \mathbb{P}\left(\mathcal{C}_{i j}\right)=x_{i j},\{i, j\} \subset\{1,2,3\}$, and $\mathbb{P}\left(\mathcal{C}_{123}\right)=x_{123}$. In particular, we show that, under logical independence, the assessment

$$
\mathcal{M}=\left(x_{1}, x_{2}, x_{3}, x_{12}, x_{13}, x_{23}, x_{123}\right)=\left(x_{1}, x_{2}, x_{3}, T_{\lambda}\left(x_{1}, x_{2}\right), T_{\lambda}\left(x_{1}, x_{3}\right), T_{\lambda}\left(x_{2}, x_{3}\right), T_{\lambda}\left(x_{1}, x_{2}, x_{3}\right)\right)
$$

on $\mathcal{F}$ is coherent for every $\left(x_{1}, x_{2}, x_{3}\right) \in[0,1]^{3}$ when $T_{\lambda}$ is the minimum t-norm $T_{M}=T_{0}$, or $T_{\lambda}$ is the product t-norm, $T_{P}=T_{1}$. Moreover, when $T_{\lambda}$ is the Lukasiewicz t-norm $T_{L}=T_{+\infty}$, the coherence of $\mathcal{M}$ is not assured. We first observe that, by Definition 4 the conjunction $\mathcal{C}_{123}=\left(E_{1} \mid H_{1}\right) \wedge\left(E_{2} \mid H_{2}\right) \wedge\left(E_{3} \mid H_{3}\right)$ is

$$
\mathrm{C}_{123}= \begin{cases}1, & \text { if } E_{1} H_{1} E_{2} H_{2} E_{3} H_{3} \text { is true } \\ 0, & \text { if } \bar{E}_{1} H_{1} \vee \bar{E}_{2} H_{2} \vee \bar{E}_{3} H_{3} \text { is true, } \\ x_{1}, & \text { if } \bar{H}_{1} E_{2} H_{2} E_{3} H_{3} \text { is true, } \\ x_{2}, & \text { if } \bar{H}_{2} E_{1} H_{1} E_{3} H_{3} \text { is true, } \\ x_{3}, & \text { if } \bar{H}_{3} E_{1} H_{1} E_{2} H_{2} \text { is true, } \\ x_{12}, & \text { if } \bar{H}_{1} \bar{H}_{2} E_{3} H_{3} \text { is true, } \\ x_{13}, & \text { if } \bar{H}_{1} \bar{H}_{3} E_{2} H_{2} \text { is true, } \\ x_{23}, & \text { if } \bar{H}_{2} \bar{H}_{3} E_{1} H_{1} \text { is true, } \\ x_{123}, & \text { if } \bar{H}_{1} \bar{H}_{2} \bar{H}_{3} \text { is true. }\end{cases}
$$

The next result characterizes the set of all coherent assessments on $\mathcal{F}([33$, Theorem 15]). 
Theorem 20. Assume that the events $E_{1}, E_{2}, E_{3}, H_{1}, H_{2}, H_{3}$ are logically independent, with $H_{1} \neq \varnothing, H_{2} \neq$ $\varnothing, H_{3} \neq \varnothing$. Then, the set $\Pi$ of all coherent assessments $\mathcal{M}=\left(x_{1}, x_{2}, x_{3}, x_{12}, x_{13}, x_{23}, x_{123}\right)$ on $\mathcal{F}=$ $\left\{\mathfrak{C}_{1}, \mathfrak{C}_{2}, \mathfrak{C}_{3}, \mathfrak{C}_{12}, \mathfrak{C}_{13}, \mathfrak{C}_{23}, \mathfrak{C}_{123}\right\}$ is the set of points $\left(x_{1}, x_{2}, x_{3}, x_{12}, x_{13}, x_{23}, x_{123}\right)$ which satisfy the following conditions

$$
\left\{\begin{array}{l}
\left(x_{1}, x_{2}, x_{3}\right) \in[0,1]^{3}, \\
\max \left\{x_{1}+x_{2}-1, x_{13}+x_{23}-x_{3}, 0\right\} \leqslant x_{12} \leqslant \min \left\{x_{1}, x_{2}\right\}, \\
\max \left\{x_{1}+x_{3}-1, x_{12}+x_{23}-x_{2}, 0\right\} \leqslant x_{13} \leqslant \min \left\{x_{1}, x_{3}\right\}, \\
\max \left\{x_{2}+x_{3}-1, x_{12}+x_{13}-x_{1}, 0\right\} \leqslant x_{23} \leqslant \min \left\{x_{2}, x_{3}\right\}, \\
1-x_{1}-x_{2}-x_{3}+x_{12}+x_{13}+x_{23} \geqslant 0, \\
x_{123} \geqslant \max \left\{0, x_{12}+x_{13}-x_{1}, x_{12}+x_{23}-x_{2}, x_{13}+x_{23}-x_{3}\right\}, \\
x_{123} \leqslant \min \left\{x_{12}, x_{13}, x_{23}, 1-x_{1}-x_{2}-x_{3}+x_{12}+x_{13}+x_{23}\right\} .
\end{array}\right.
$$

Remark 8. As it can be verified, the last two inequalities in (73), that is

$$
\begin{aligned}
& \max \left\{0, x_{12}+x_{13}-x_{1}, x_{12}+x_{23}-x_{2}, x_{13}+x_{23}-x_{3}\right\} \leqslant x_{123} \leqslant \\
& \leqslant \min \left\{x_{12}, x_{13}, x_{23}, 1-x_{1}-x_{2}-x_{3}+x_{12}+x_{13}+x_{23}\right\},
\end{aligned}
$$

imply all the other inequalities in (73). For instance, in order to prove that $x_{1} \leqslant 1$, we observe that from (74) it holds that

$$
x_{12}+x_{23}-x_{2} \leqslant 1-x_{1}-x_{2}-x_{3}+x_{12}+x_{13}+x_{23},
$$

from which it follows

$$
x_{12}+x_{23}-x_{2}+x_{2}+x_{3}-x_{12}-x_{13}-x_{23} \leqslant 1-x_{1},
$$

that is $x_{3}-x_{13} \leqslant 1-x_{1}$. Moreover, still from (74), it holds that $x_{13}+x_{23}-x_{3} \leqslant x_{23}$, that is $x_{3}-x_{13} \geqslant$ 0 . Thus $x_{1} \leqslant 1$. Then, as (73) and (74) are equivalent, the set $\Pi$ in Theorem 20 is the set of points $\left(x_{1}, x_{2}, x_{3}, x_{12}, x_{13}, x_{23}, x_{123}\right)$ which satisfy (74).

Then, by Theorem 20 it follows [33, Corollary 1]

Corollary 1. For any coherent assessment $\left(x_{1}, x_{2}, x_{3}, x_{12}, x_{13}, x_{23}\right)$ on $\left\{\mathfrak{C}_{1}, \mathfrak{C}_{2}, \mathfrak{C}_{3}, \mathfrak{C}_{12}, \mathfrak{C}_{13}, \mathfrak{C}_{23}\right\}$ the extension $x_{123}$ on $\mathcal{C}_{123}$ is coherent if and only if $x_{123} \in\left[x_{123}^{\prime}, x_{123}^{\prime \prime}\right]$, where

$$
\begin{aligned}
& x_{123}^{\prime}=\max \left\{0, x_{12}+x_{13}-x_{1}, x_{12}+x_{23}-x_{2}, x_{13}+x_{23}-x_{3}\right\}, \\
& x_{123}^{\prime \prime}=\min \left\{x_{12}, x_{13}, x_{23}, 1-x_{1}-x_{2}-x_{3}+x_{12}+x_{13}+x_{23}\right\} .
\end{aligned}
$$

We recall that in case of logical dependencies, the set of all coherent assessments may be a strict subset of the set $\Pi$ associated with the case of logical independence. However, the next result shows that the set of coherent assessments is still $\Pi$ in the case where $H_{1}=H_{2}=H_{3}=H$ (with possibly $H=\Omega$, see also [38, p. 232]).

Theorem 21. Let be given any logically independent events $E_{1}, E_{2}, E_{3}, H$, with $H \neq \varnothing$. Then, the set $\Pi$ of all coherent assessments $\mathcal{M}=\left(x_{1}, x_{2}, x_{3}, x_{12}, x_{13}, x_{23}, x_{123}\right)$ on $\mathcal{F}=\left\{\mathfrak{C}_{1}, \mathfrak{C}_{2}, \mathfrak{C}_{3}, \mathfrak{C}_{12}, \mathfrak{C}_{13}, \mathfrak{C}_{23}, \mathfrak{C}_{123}\right\}$ is the set of points $\left(x_{1}, x_{2}, x_{3}, x_{12}, x_{13}, x_{23}, x_{123}\right)$ which satisfy the conditions in formula (73).

A corollary similar to Corollary 11 could be associated to Theorem 21. For a similar result based on copulas see [21].

In the next subsection we examine the coherence of the prevision assessment $\mathcal{M}=$ $\left(x_{1}, x_{2}, x_{3}, T_{\lambda}\left(x_{1}, x_{2}\right), T_{\lambda}\left(x_{1}, x_{3}\right), T_{\lambda}\left(x_{2}, x_{3}\right), T_{\lambda}\left(x_{1}, x_{2}, x_{3}\right)\right)$ on $\mathcal{F}=\left\{\mathfrak{C}_{1}, \mathfrak{C}_{2}, \mathfrak{C}_{3}, \mathfrak{C}_{12}, \mathfrak{C}_{13}, \mathfrak{C}_{23}, \mathfrak{C}_{123}\right\}$ in the cases where $T_{\lambda}$ is the minimum t-norm, or the product t-norm, or the Lukasiewicz t-norm. 


\subsection{On the minimum $t$-norm}

We recall that the Frank t-norm $T_{0}$ is the minimum t-norm $T_{M}$.

Theorem 22. Assume that the events $E_{1}, E_{2}, E_{3}, H_{1}, H_{2}, H_{3}$ are logically independent, with $H_{1} \neq$ $\varnothing, H_{2} \neq \varnothing, H_{3} \neq \varnothing$. The assessment $\mathcal{M}=\left(x_{1}, x_{2}, x_{3}, T_{M}\left(x_{1}, x_{2}\right), T_{M}\left(x_{1}, x_{3}\right), T_{M}\left(x_{2}, x_{3}\right), T_{M}\left(x_{1}, x_{2}, x_{3}\right)\right)$ on $\mathcal{F}=\left\{\mathfrak{C}_{1}, \mathfrak{C}_{2}, \mathfrak{C}_{3}, \mathfrak{C}_{12}, \mathfrak{C}_{13}, \mathfrak{C}_{23}, \mathfrak{C}_{123}\right\}$, with $\left(x_{1}, x_{2}, x_{3}\right) \in[0,1]^{3}$, is coherent. Moreover, when $\mathcal{M}=\left(x_{1}, x_{2}, x_{3}, T_{M}\left(x_{1}, x_{2}\right), T_{M}\left(x_{1}, x_{3}\right), T_{M}\left(x_{2}, x_{3}\right), T_{M}\left(x_{1}, x_{2}, x_{3}\right)\right)$, it holds that $\mathcal{C}_{i j}=T_{M}\left(\mathcal{C}_{i}, \mathcal{C}_{j}\right)=$ $\min \left\{\mathrm{C}_{i}, \mathrm{C}_{j}\right\}, i \neq j$, and $\mathrm{C}_{123}=T_{M}\left(\mathrm{C}_{1}, \mathrm{C}_{2}, \mathrm{C}_{3}\right)=\min \left\{\mathrm{C}_{1}, \mathrm{e}_{2}, \mathrm{C}_{3}\right\}$.

Proof. From Remark 8 , the coherence of $\mathcal{M}$ amounts to the inequalities in (74). Without loss of generality, we assume that $0 \leqslant x_{1} \leqslant x_{2} \leqslant x_{3} \leqslant 1$. Then $x_{12}=T_{M}\left(x_{1}, x_{2}\right)=x_{1}, x_{13}=T_{M}\left(x_{1}, x_{3}\right)=x_{1}$, $x_{23}=T_{M}\left(x_{2}, x_{3}\right)=x_{2}$, and $x_{123}=T_{M}\left(x_{1}, x_{2}, x_{3}\right)=x_{1}$. The inequalities (74) become

$$
\max \left\{0, x_{1}, x_{1}+x_{2}-x_{3}\right\}=x_{1} \leqslant x_{1} \leqslant x_{1}=\min \left\{x_{1}, x_{2}, 1-x_{3}+x_{1}\right\} .
$$

Thus, the inequalities are satisfied and hence $\mathcal{M}$ is coherent. By Remark 6 , it holds that $\mathcal{C}_{i j}=T_{M}\left(\mathcal{C}_{i}, \mathcal{C}_{j}\right)=$ $\min \left\{\mathfrak{C}_{i}, \mathfrak{C}_{j}\right\}, i \neq j$. Moreover, based on (72), it can be easily verified that $\mathfrak{C}_{123}=T_{M}\left(\mathfrak{C}_{1}, \mathfrak{C}_{2}, \mathfrak{C}_{3}\right)=$ $\min \left\{\mathfrak{C}_{1}, \mathfrak{C}_{2}, \mathfrak{C}_{3}\right\}$.

Remark 9. As we can see from (76) and Corollary 10 the assessment $x_{123}=\min \left\{x_{1}, x_{2}, x_{3}\right\}$ is the unique coherent extension on $\mathcal{C}_{123}$ of the assessment $\left(x_{1}, x_{2}, x_{3}, \min \left\{x_{1}, x_{2}\right\}, \min \left\{x_{1}, x_{3}\right\}, \min \left\{x_{2}, x_{3}\right\}\right)$ on $\left\{\mathrm{C}_{1}, \mathrm{C}_{2}, \mathrm{e}_{3}, \mathrm{C}_{12}, \mathrm{e}_{13}, \mathrm{C}_{23}\right\}$.

\subsection{On the Product t-norm}

We recall that the Frank t-norm $T_{1}$ is the product t-norm $T_{P}$.

Theorem 23. Assume that the events $E_{1}, E_{2}, E_{3}, H_{1}, H_{2}, H_{3}$ are logically independent, with $H_{1} \neq \varnothing, H_{2} \neq$ $\varnothing, H_{3} \neq \varnothing$. The assessment $\mathcal{M}=\left(x_{1}, x_{2}, x_{3}, T_{P}\left(x_{1}, x_{2}\right), T_{P}\left(x_{1}, x_{3}\right), T_{P}\left(x_{2}, x_{3}\right), T_{P}\left(x_{1}, x_{2}, x_{3}\right)\right)$ on $\mathcal{F}=\left\{\mathfrak{C}_{1}, \mathfrak{C}_{2}, \mathfrak{C}_{3}, \mathfrak{C}_{12}, \mathfrak{C}_{13}, \mathfrak{C}_{23}, \mathfrak{C}_{123}\right\}$, with $\left(x_{1}, x_{2}, x_{3}\right) \in[0,1]^{3}$, is coherent. Moreover, when $\mathcal{M}=$ $\left(x_{1}, x_{2}, x_{3}, T_{P}\left(x_{1}, x_{2}\right), T_{P}\left(x_{1}, x_{3}\right), T_{P}\left(x_{2}, x_{3}\right), T_{P}\left(x_{1}, x_{2}, x_{3}\right)\right)$, it holds that $\mathcal{C}_{i j}=T_{P}\left(\mathcal{C}_{i}, \mathcal{C}_{j}\right)=\mathcal{C}_{i} \mathfrak{C}_{j}, i \neq j$, and $\mathfrak{C}_{123}=T_{P}\left(\mathcal{C}_{1}, \mathfrak{C}_{2}, \mathfrak{C}_{3}\right)=\mathfrak{C}_{1} \mathfrak{C}_{2} \mathfrak{C}_{3}$.

Proof. From Remark 8 , the coherence of $\mathcal{M}$ amounts to the inequalities in (74). As $x_{i j}=T_{P}\left(x_{i}, x_{j}\right)=x_{i} x_{j}$, $i \neq j$, and $x_{123}=T_{P}\left(x_{1}, x_{2}, x_{3}\right)=x_{1} x_{2} x_{3}$, the inequalities (74) become

$$
\begin{aligned}
& \max \left\{0, x_{1}\left(x_{2}+x_{3}-1\right), x_{2}\left(x_{1}+x_{3}-1\right), x_{3}\left(x_{1}+x_{2}-1\right)\right\} \leqslant x_{1} x_{2} x_{3} \leqslant \\
& \leqslant \min \left\{x_{1} x_{2}, x_{1} x_{3}, x_{2} x_{3},\left(1-x_{1}\right)\left(1-x_{2}\right)\left(1-x_{3}\right)+x_{1} x_{2} x_{3}\right\} .
\end{aligned}
$$

As $\left(x_{1}, x_{2}, x_{3}\right) \in[0,1]^{3}$ it holds that $x_{i}+x_{j}-1 \leqslant x_{i} x_{j}$ because $x_{i}\left(1-x_{j}\right) \leqslant 1-x_{j}$. Then, the first inequality in (77) is satisfied. Moreover, the second inequality is trivial. Thus, $\mathcal{M}$ is coherent. By Remark 6, it holds that $\mathcal{C}_{i j}=T_{P}\left(\mathcal{C}_{i}, \mathcal{C}_{j}\right)=\min \left\{\mathcal{C}_{i}, \mathcal{C}_{j}\right\}, i \neq j$. Finally, based on (72), it can be easily verified that $\mathcal{C}_{123}=T_{P}\left(\mathcal{C}_{1}, \mathcal{C}_{2}, \mathcal{C}_{3}\right)=\mathcal{C}_{1} \mathcal{C}_{2} \mathfrak{C}_{3}$.

\subsection{On Lukasiewicz t-norm}

We recall that the Frank t-norm $T_{+\infty}$ is the Lukasiewicz t-norm $T_{L}$. We show that the assessment $\mathcal{M}=\left(x_{1}, x_{2}, x_{3}, T_{L}\left(x_{1}, x_{2}\right), T_{L}\left(x_{1}, x_{3}\right), T_{L}\left(x_{2}, x_{3}\right), T_{L}\left(x_{1}, x_{2}, x_{3}\right)\right)$ on $\mathcal{F}=\left\{\mathfrak{C}_{1}, \mathfrak{C}_{2}, \mathfrak{C}_{3}, \mathfrak{C}_{12}, \mathfrak{C}_{13}, \mathfrak{C}_{23}, \mathfrak{C}_{123}\right\}$ may be not coherent for some $\left(x_{1}, x_{2}, x_{3}\right) \in[0,1]^{3}$, as shown in the example below. 
Example 7. Given any logically independent events $E_{1}, E_{2}, E_{3}, H_{1}, H_{2}, H_{3}$, the assessment $\left(x_{1}, x_{2}, x_{3}\right)=(0.5,0.6,0.7)$ on $\left\{\mathfrak{C}_{1}, \mathfrak{C}_{2}, \mathfrak{C}_{3}\right\}$ is coherent. However, the prevision assessment $\left(x_{1}, x_{2}, x_{3}, T_{L}\left(x_{1}, x_{2}\right), T_{L}\left(x_{1}, x_{3}\right), T_{L}\left(x_{2}, x_{3}\right), T_{L}\left(x_{1}, x_{2}, x_{3}\right)\right)=(0.5,0.6,0.7,0.1,0.2,0.3,0)$ on the family $\mathcal{F}=\left\{\mathfrak{C}_{1}, \mathfrak{C}_{2}, \mathfrak{C}_{3}, \mathfrak{C}_{12}, \mathfrak{C}_{13}, \mathfrak{C}_{23}, \mathfrak{C}_{123}\right\}$ is not coherent. Indeed, formula (74) becomes

$\max \{0,0.1+0.2-0.5,0.1+0.3-0.6,0.2+0.3-0.7\} \leqslant 0 \leqslant \min \{0.1,0.2,0.3,1-0.5-0.6-0.7+0.1+0.2+0.3\}$,

that is:

$$
\max \{0,-0.2\}=0 \leqslant 0 \leqslant-0.2=\min \{0.1,0.2,0.3,-0.2\} ;
$$

thus, the inequalities in (74) are not satisfied and by Remark 8 the assessment $(0.5,0.6,0.7,0.1,0.2,0.3,0)$ is not coherent. Then, the results of Theorems 22 and 23 do not hold for the Lukasiewicz t-norm.

In the next result we illustrate further details on coherence of the prevision assessment $\left(x_{1}, x_{2}, x_{3}, T_{L}\left(x_{1}, x_{2}\right), T_{L}\left(x_{1}, x_{3}\right), T_{L}\left(x_{2}, x_{3}\right), T_{L}\left(x_{1}, x_{2}, x_{3}\right)\right)$.

Theorem 24. Assume that the events $E_{1}, E_{2}, E_{3}, H_{1}, H_{2}, H_{3}$ are logically independent, with $H_{1} \neq \varnothing, H_{2} \neq$ $\varnothing, H_{3} \neq \varnothing$. Let $\mathcal{M}=\left(x_{1}, x_{2}, x_{3}, T_{L}\left(x_{1}, x_{2}\right), T_{L}\left(x_{1}, x_{3}\right), T_{L}\left(x_{2}, x_{3}\right), T_{L}\left(x_{1}, x_{2}, x_{3}\right)\right)$ be a prevision assessment on the family $\mathcal{F}=\left\{\mathfrak{C}_{1}, \mathfrak{C}_{2}, \mathfrak{C}_{3}, \mathfrak{C}_{12}, \mathfrak{C}_{13}, \mathfrak{C}_{23}, \mathfrak{C}_{123}\right\}$. If $\left(x_{1}, x_{2}, x_{3}\right) \in[0,1]^{3}$ and $x_{1}+x_{2}+x_{3}-2 \geqslant 0$ then $\mathcal{M}$ is coherent. If $\left(x_{1}, x_{2}, x_{3}\right) \in[0,1]^{3}, x_{1}+x_{2}-1>0, x_{1}+x_{3}-1>0, x_{2}+x_{3}-1>0$, and $x_{1}+x_{2}+x_{3}-2<0$, then $\mathcal{M}$ is not coherent.

Proof. We observe that the set of points $\left(x_{1}, x_{2}, x_{3}\right) \in[0,1]^{3}$ such that $x_{1}+x_{2}+x_{3}-2 \geqslant 0$ is the convex hull $\mathcal{T}$ of the points $(1,1,0),(1,0,1),(0,1,1),(1,1,1)$, which is a tetrahedron. If $\left(x_{1}, x_{2}, x_{3}\right) \in \mathcal{T}$, then $x_{1}+x_{2}+x_{3}-2 \geqslant 0$, and it holds that

$$
x_{1}+x_{2}-1 \geqslant 0, \quad x_{1}+x_{3}-1 \geqslant 0, \quad x_{2}+x_{3}-1 \geqslant 0,
$$

with

$$
0 \leqslant x_{1}+x_{2}+x_{3}-2 \leqslant \min \left\{x_{1}+x_{2}-1, x_{1}+x_{3}-1, x_{2}+x_{3}-1\right\} .
$$

Thus, the assessment becomes $\mathcal{M}=\left(x_{1}, x_{2}, x_{3}, x_{1}+x_{2}-1, x_{1}+x_{3}-1, x_{2}+x_{3}-1, x_{1}+x_{2}+x_{3}-2\right)$. Moreover, from (78), the conditions of coherence on $\mathcal{M}$ given in (74) become

$\max \left\{0, x_{1}+x_{2}+x_{3}-2\right\} \leqslant x_{1}+x_{2}+x_{3}-2 \leqslant \min \left\{x_{1}+x_{2}-1, x_{1}+x_{3}-1, x_{2}+x_{3}-1, x_{1}+x_{2}+x_{3}-2\right\}$,

that is

$$
x_{1}+x_{2}+x_{3}-2 \leqslant x_{1}+x_{2}+x_{3}-2 \leqslant x_{1}+x_{2}+x_{3}-2,
$$

which are trivially satisfied. Then, by Remark $8, \mathcal{M}$ is coherent. then

$$
\text { If }\left(x_{1}, x_{2}, x_{3}\right) \in[0,1]^{3}, x_{1}+x_{2}-1>0, x_{1}+x_{3}-1>0, x_{2}+x_{3}-1>0 \text {, and } x_{1}+x_{2}+x_{3}-2<0 \text {, }
$$

$$
1-x_{1}-x_{2}-x_{3}+x_{12}+x_{13}+x_{23}=1-x_{1}-x_{2}-x_{3}+T_{L}\left(x_{1}, x_{2}\right)+T_{L}\left(x_{1}, x_{3}\right)+T_{L}\left(x_{2}, x_{3}\right) ;
$$

moreover

$$
1-x_{1}-x_{2}-x_{3}+T_{L}\left(x_{1}, x_{2}\right)+T_{L}\left(x_{1}, x_{3}\right)+T_{L}\left(x_{2}, x_{3}\right)=x_{1}+x_{2}+x_{3}-2<0 .
$$

Then the inequality $1-x_{1}-x_{2}-x_{3}+x_{12}+x_{13}+x_{23} \geqslant 0$ in (74) is not satisfied. Therefore, by Remark 8. $\mathcal{M}$ is not coherent. This is the case, for instance, in Example 7 . 
Remark 10. Notice that, if we consider the assessment $\left(x_{1}, x_{2}, x_{3}, T_{L}\left(x_{1}, x_{2}\right), T_{L}\left(x_{1}, x_{3}\right), T_{L}\left(x_{2}, x_{3}\right), x_{123}\right)$ on the family $\left\{\mathfrak{C}_{1}, \mathfrak{C}_{2}, \mathfrak{C}_{3}, \mathfrak{C}_{12}, \mathfrak{C}_{13}, \mathfrak{C}_{23}, \mathfrak{C}_{123}\right\}$, under the condition $x_{1}+x_{2}+x_{3}-2 \geqslant 0$, the conditions of coherence given in (74) become

$$
\max \left\{0, x_{1}+x_{2}+x_{3}-2\right\} \leqslant x_{123} \leqslant \min \left\{x_{1}+x_{2}-1, x_{1}+x_{3}-1, x_{2}+x_{3}-1, x_{1}+x_{2}+x_{3}-2\right\},
$$

that is the conditions of coherence on $\mathcal{M}$ given in (74) become

$$
x_{1}+x_{2}+x_{3}-2 \leqslant x_{123} \leqslant x_{1}+x_{2}+x_{3}-2 .
$$

Thus, the unique coherent extension on $\mathcal{C}_{123}$ is $x_{123}=x_{1}+x_{2}+x_{3}-2=T_{L}\left(x_{1}, x_{2}, x_{3}\right)$. In this case, it holds that $\mathfrak{C}_{i j}=T_{L}\left(\mathfrak{C}_{i}, \mathcal{C}_{j}\right)=\mathcal{C}_{i}+\mathcal{C}_{j}-1, i \neq j$, and $\mathfrak{C}_{123}=T_{L}\left(\mathcal{C}_{1}, \mathcal{C}_{2}, \mathcal{C}_{3}\right)=\mathfrak{C}_{1}+\mathfrak{C}_{2}+\mathfrak{C}_{3}-2$.

Finally, we point out again that when $T_{\lambda}$ is the Lukasiewicz t-norm $T_{L}=T_{+\infty}$, it may happen that the assessment $\mathcal{M}=\left(x_{1}, x_{2}, x_{3}, T_{L}\left(x_{1}, x_{2}\right), T_{L}\left(x_{1}, x_{3}\right), T_{L}\left(x_{2}, x_{3}\right), T_{L}\left(x_{1}, x_{2}, x_{3}\right)\right)$ is not coherent, that is for some values $x_{1}, x_{2}$, and $x_{3}$, the assessment $\mathcal{M}$, with $x_{12}=T_{L}\left(x_{1}, x_{2}\right), x_{13}=T_{L}\left(x_{1}, x_{3}\right), x_{23}=T_{L}\left(x_{2}, x_{3}\right)$, and $x_{123}=T_{L}\left(x_{1}, x_{2}, x_{3}\right)$, is not coherent. Then, to assign conditional previsions by means of Lukasiewicz t-norm may be inconsistent. In Theorem 24 we gave some sufficient conditions for coherence/incoherence of $\mathcal{M}$ when using $T_{L}$.

\section{Conclusions}

In this paper we studied conjoined and disjoined conditionals, Frank t-norms and t-conorms, and the sharpness of Fréchet-Hoeffding bounds. By studying the solvability of suitable linear systems, we showed that, under logical independence, the Fréchet-Hoeffding bounds for the prevision of the conjunction and the disjunction of $n$ conditional events are sharp. In particular we illustrated some details in the case $n=3$. We gave a geometrical characterization of the set $\Pi$ of all coherent prevision assessments on $\left\{E_{1}\left|H_{1}, \ldots, E_{n}\right| H_{n}, \mathcal{C}_{1 \ldots n}\right\}$, by verifying that $\Pi$ is convex. We discussed the case where previsions of conjunctions are assessed by Lukasiewicz t-norms and we found explicit solutions for the relevant linear systems; then, we analyzed a selected example. We studied the representation of the prevision of $\mathcal{C}_{1 \cdots n}$ and $\mathcal{D}_{1 \cdots n}$ by a Frank t-norm $T_{\lambda}$ and a Frank t-conorm $S_{\lambda}$, respectively. Then, we characterized the sets of coherent prevision assessments on $\left\{E_{1}\left|H_{1}, \ldots, E_{n}\right| H_{n}, \mathcal{C}_{1 \ldots n}\right\}$ and on $\left\{E_{1}\left|H_{1}, \ldots, E_{n}\right| H_{n}, \mathcal{D}_{1 \ldots n}\right\}$ by using $T_{\lambda}$ and $S_{\lambda}$. We showed that, under logical independence, $T_{\lambda}(A|H, B| K)$ is a conjunction $(A \mid H) \wedge(B \mid K)$ and $S_{\lambda}(A|H, B| K)$ is a disjunction $(A \mid H) \vee(B \mid K)$, for every $\lambda \in[0,+\infty]$. We also examined the case of logical dependence where $A=B$, by obtaining the set of coherent assessments on $A|H, A| K,(A \mid H) \wedge(A \mid K)$ and its representation in terms of $T_{\lambda}$, with $\lambda \in[0,1]$. We obtained some particular results on Frank t-norms and coherence of prevision assessments on the family $\mathcal{F}=\left\{\mathfrak{C}_{1}, \mathfrak{C}_{2}, \mathfrak{C}_{3}, \mathfrak{C}_{12}, \mathfrak{C}_{13}, \mathfrak{C}_{23}, \mathfrak{C}_{123}\right\}$. In particular, we verified that, under logical independence, the assessment $\mathcal{M}=\left(x_{1}, x_{2}, x_{3}, T_{\lambda}\left(x_{1}, x_{2}\right), T_{\lambda}\left(x_{1}, x_{3}\right), T_{\lambda}\left(x_{2}, x_{3}\right), T_{\lambda}\left(x_{1}, x_{2}, x_{3}\right)\right)$ on $\mathcal{F}$ is coherent for every $\left(x_{1}, x_{2}, x_{3}\right) \in[0,1]^{3}$ when $T_{\lambda}$ is the minimum t-norm $T_{M}$, or the product $\mathrm{t}$-norm $T_{P}$. We showed that in these cases the conjunction $\mathcal{C}_{123}$ coincides with $T_{M}\left(\mathfrak{C}_{1}, \mathcal{C}_{2}, \mathcal{C}_{3}\right)$, or $T_{P}\left(\mathfrak{C}_{1}, \mathfrak{C}_{2}, \mathfrak{C}_{3}\right)$, respectively. Based on a counterexample, we verified that, when $T_{\lambda}$ is the Lukasiewicz t-norm $T_{L}$, the coherence of $\mathcal{M}$ is not assured. Then, we remarked that the Lukasiewicz t-norm of three conditional events may not be a conjunction. Finally, we gave two sufficient conditions for coherence and incoherence of $\mathcal{M}$, respectively, when using the Lukasiewicz t-norm. Future work could concern possible applications to fuzzy logic in the setting of coherence (see, e.g., [8, 13, 14]) by interpreting multidimensional membership functions as previsions of conjunctions of conditional events. 


\section{Declaration of competing interest}

We wish to confirm that there are no known conflicts of interest associated with this publication and there has been no significant financial support for this work that could have influenced its outcome.

\section{Acknowledgments}

We thank the anonymous reviewers for their comments and suggestions which were very helpful in improving this paper. Giuseppe Sanfilippo has been partially supported by the INdAM-GNAMPA Project (2020 Grant U-UFMBAZ-2020-000819).

\section{References}

[1] Batyrshin, I., Kaynak, O., Rudas, I., 2002. Fuzzy modeling based on generalized conjunction operations. IEEE Transactions on Fuzzy Systems 10, 678-683. doi 10.1109/TFUZZ.2002.803500

[2] Berti, P., Miranda, E., Rigo, P., 2017. Basic ideas underlying conglomerability and disintegrability. International Journal of Approximate Reasoning 88, 387 - 400. doi https://doi.org/10.1016/j.ijar.2017.06.009

[3] Biazzo, V., Gilio, A., Lukasiewicz, T., Sanfilippo, G., 2005. Probabilistic logic under coherence: Complexity and algorithms. Annals of Mathematics and Artificial Intelligence 45, 35-81. doi https://doi.org/10.1007/s10472-005-9005-y

[4] Biazzo, V., Gilio, A., Sanfilippo, G., 2012. Coherent conditional previsions and proper scoring rules, in: Greco, S., Bouchon-Meunier, B., Coletti, G., Fedrizzi, M., Matarazzo, B., Yager, R.R. (Eds.), Advances in Computational Intelligence. IPMU 2012 Part IV. Springer Heidelberg. volume 300 of CCIS, pp. 146-156. doi https://doi .org/10.1007/978-3-642-31724-8_16.

[5] Brozzi, A., Capotorti, A., Vantaggi, B., 2012. Incoherence correction strategies in statistical matching. Int. J. Approx. Reasoning 53, 1124 - 1136. doi/https://doi.org/10.1016/j.ijar.2012.06.009

[6] Calabrese, P., 2017. Logic and Conditional Probability: A Synthesis. College Publications.

[7] Capotorti, A., Lad, F., Sanfilippo, G., 2007. Reassessing accuracy rates of median decisions. The American Statistician 61, 132-138. doi http://dx.doi.org/10.1198/000313007X190943

[8] Coletti, G., Gervasi, O., Tasso, S., Vantaggi, B., 2012. Generalized bayesian inference in a fuzzy context: From theory to a virtual reality application. Computational Statistics and Data Analysis 56, 967 - 980. doi http://dx.doi.org/10.1016/j.csda.2011.06.020

[9] Coletti, G., Petturiti, D., Vantaggi, B., 2017. Fuzzy memberships as likelihood functions in a possibilistic framework. International Journal of Approximate Reasoning 88, 547 - 566. doi $10.1016 / j$. ijar.2016.11.017

[10] Coletti, G., Petturiti, D., Vantaggi, B., 2020. A dutch book coherence condition for conditional completely alternating choquet expectations. Bollettino dell'Unione Matematica Italiana 13, 585-593. doi 10.1007/s40574-020-00251-8

[11] Coletti, G., Scozzafava, R., 1999. Conditioning and inference in intelligent systems. Soft Computing 3, 118-130. doi https://doi .org/10.1007/s005000050060

[12] Coletti, G., Scozzafava, R., 2002. Probabilistic logic in a coherent setting. Kluwer, Dordrecht.

[13] Coletti, G., Scozzafava, R., 2004. Conditional probability, fuzzy sets, and possibility: A unifying view. Fuzzy Sets and Systems 144, 227-249. doi https://doi.org/10.1016/j.fss.2003.10.022

[14] Coletti, G., Scozzafava, R., 2006. Conditional probability and fuzzy information. Computational Statistics and Data Analysis $51,115-132$.

[15] Coletti, G., Vantaggi, B., 2018. Coherent conditional plausibility: A tool for handling fuzziness and uncertainty under partial information, in: Collan, M., Kacprzyk, J. (Eds.), Soft Computing Applications for Group Decision-making and Consensus Modeling. Springer International Publishing, Cham, pp. 129-152. doi 10.1007/978-3-319-60207-3_9

[16] Di Zio, M., Vantaggi, B., 2017. Partial identification in statistical matching with misclassification. International Journal of Approximate Reasoning 82, $227-241$.

[17] D’Orazio, M., Di Zio, M., Scanu, M., 2006. Statistical Matching: Theory and Practice. Wiley, New York.

[18] Dubois, D., Faux, F., Prade, H., 2020. Prejudice in uncertain information merging: Pushing the fusion paradigm of evidence theory further. International Journal of Approximate Reasoning 121, 1 - 22. doi $10.1016 / \mathrm{j}$. ijar.2020.02.012

[19] Dubois, D., Liu, W., Ma, J., Prade, H., 2016. The basic principles of uncertain information fusion. an organised review of merging rules in different representation frameworks. Information Fusion $32,12-39$. doi $10.1016 / j$.inffus.2016.02.006 
[20] Dujmović, J.J., Legind Larsen, H., 2007. Generalized conjunction/disjunction. International Journal of Approximate Reasoning 46, 423 - 446. doi https://doi.org/10.1016/j.ijar.2006.12.011 special Section: Aggregation Operators.

[21] Durante, F., Klement, E.P., Quesada-Molina, J.J., 2008. Bounds for trivariate copulas with given bivariate marginals. Journal of Inequalities and Applications 2008, 9 pages. Article ID 161537.

[22] Flaminio, T., Godo, L., Hosni, H., 2020. Boolean algebras of conditionals, probability and logic. Artificial Intelligence 286, 103347. doi $10.1016 / \mathrm{j}$. artint.2020.103347

[23] Gale, D., 1960. The theory of linear economic models. McGraw-Hill, New York.

[24] Gilio, A., 1993. Probabilistic consistency of knowledge bases in inference systems, in: Clarke, M., Kruse, R., Moral, S. (Eds.), Symbolic and Quantitative Approaches to Reasoning and Uncertainty. Springer, Berlin, Heidelberg. volume 747 of LNCS, pp. 160-167.

[25] Gilio, A., Ingrassia, S., 1998. Totally coherent set-valued probability assessments. Kybernetika 34, 3-15.

[26] Gilio, A., Pfeifer, N., Sanfilippo, G., 2016. Transitivity in coherence-based probability logic. Journal of Applied Logic 14, 46-64. doi https://doi.org/10.1016/j.jal.2015.09.012

[27] Gilio, A., Pfeifer, N., Sanfilippo, G., 2020. Probabilistic entailment and iterated conditionals, in: Elqayam, S., Douven, I., Evans, J.S.B.T., Cruz, N. (Eds.), Logic and Uncertainty in the Human Mind: A Tribute to David E. Over. Routledge, Oxon, pp. 71-101. doi https://doi.org/10.4324/9781315111902-6

[28] Gilio, A., Sanfilippo, G., 2013a. Conditional random quantities and iterated conditioning in the setting of coherence, in: van der Gaag, L.C. (Ed.), ECSQARU 2013. Springer, Berlin, Heidelberg. volume 7958 of LNCS, pp. 218-229. doi $10.1007 / 978-3-642-39091-3 \_19$

[29] Gilio, A., Sanfilippo, G., 2013b. Conjunction, disjunction and iterated conditioning of conditional events, in: Synergies of Soft Computing and Statistics for Intelligent Data Analysis. Springer, Berlin. volume 190 of AISC, pp. 399-407. doi $10.1007 / 978-3-642-33042-1 \_43$

[30] Gilio, A., Sanfilippo, G., 2014. Conditional random quantities and compounds of conditionals. Studia Logica 102, 709-729. doi $10.1007 / \mathrm{s} 11225-013-9511-6$.

[31] Gilio, A., Sanfilippo, G., 2017. Conjunction and disjunction among conditional events, in: Benferhat, S., Tabia, K., Ali, M. (Eds.), IEA/AIE 2017, Part II. Springer, Cham. volume 10351 of LNCS, pp. 85-96. doi 10.1007/978-3-319-60045-1_11.

[32] Gilio, A., Sanfilippo, G., 2019a. Conjunction of conditional events and t-norms, in: Kern-Isberner, G., Ognjanović, Z. (Eds.), Symbolic and Quantitative Approaches to Reasoning with Uncertainty, ECSQARU 2019. Springer International Publishing. volume 11726 of LNCS, pp. 199-211. doi 10.1007/978-3-030-29765-7_17

[33] Gilio, A., Sanfilippo, G., 2019b. Generalized logical operations among conditional events. Applied Intelligence 49, 79-102. doi $10.1007 / \mathrm{s} 10489-018-1229-8$.

[34] Gilio, A., Sanfilippo, G., 2020. Algebraic aspects and coherence conditions for conjoined and disjoined conditionals. International Journal of Approximate Reasoning 126, 98 - 123. doi https://doi.org/10.1016/j.ijar.2020.08.004

[35] Gilio, A., Sanfilippo, G., To Appear. On compound and iterated conditionals. Argumenta .

[36] Grabisch, M., Marichal, J., Mesiar, R., Pap, E., 2009. Aggregation functions. Cambridge University Press.

[37] Holzer, S., 1985. On coherence and conditional prevision. Bollettino dell'Unione Matematica Italiana 4, 441-460.

[38] Joe, H., 1997. Multivariate Models and Multivariate Dependence Concepts. Chapman and Hall/CRC, New York.

[39] Kaufmann, S., 2009. Conditionals right and left: Probabilities for the whole family. Journal of Philosophical Logic 38, 1-53. doi $10.1007 / \mathrm{s} 10992-008-9088-0$

[40] Klement, E., Mesiar, R., Pap, E., 2005. Triangular norms: Basic notions and properties, in: Klement, E., Mesiar, R. (Eds.), Logical, Algebraic, Analytic and Probabilistic Aspects of Triangular Norms. Elsevier Science B.V., Amsterdam, pp. 17 - 60.

[41] Klement, E.P., Mesiar, R., Pap, E., 2000. Triangular Norms. Springer.

[42] Lad, F., 1996. Operational subjective statistical methods: A mathematical, philosophical, and historical introduction. Wiley, New York.

[43] Lad, F., Sanfilippo, G., 2020. Predictive distributions that mimic frequencies over a restricted subdomain. Decisions in Economics and Finance 43, 17-41. doi https://doi.org/10.1007/s10203-020-00281-z

[44] McGee, V., 1989. Conditional probabilities and compounds of conditionals. Philosophical Review 98, 485-541.

[45] Mundici, D., 2021. Deciding koopman's qualitative probability. Artificial Intelligence 299, 103524. URL: https://www.sciencedirect.com/science/article/pii/S0004370221000758. doi https://doi.org/10.1016/j.artint.2021.103524

[46] Petturiti, D., Vantaggi, B., 2017. Envelopes of conditional probabilities extending a strategy and a prior probability. International Journal of Approximate Reasoning 81, 160 - 182.

[47] Petturiti, D., Vantaggi, B., 2020. Modeling agent's conditional preferences under objective ambiguity in dempster-shafer theory. International Journal of Approximate Reasoning 119, 151-176. doi https://doi.org/10.1016/j.ijar.2019.12.019

[48] Pfeifer, N., Sanfilippo, G., 2017. Probabilistic squares and hexagons of opposition under coherence. International Journal of 
Approximate Reasoning 88, 282-294. doi 10.1016/j.ijar.2017.05.014

[49] Rawal, R., Goel, K., Gupta, C., 2020. Covid-19: Disease pattern study based on semantic-web approach using description logic, in: 2020 IEEE International Conference for Innovation in Technology (INOCON), pp. 1-5. doi $10.1109 /$ INOCON50539.2020.9298278.

[50] Regazzini, E., 1985. Finitely additive conditional probabilities. Rendiconti del Seminario Matematico e Fisico di Milano 55, 69-89.

[51] Sanfilippo, G., 2018. Lower and upper probability bounds for some conjunctions of two conditional events, in: SUM 2018. Springer International Publishing, Cham. volume 11142 of LNCS, pp. 260-275.

[52] Sanfilippo, G., Gilio, A., Over, D., Pfeifer, N., 2020. Probabilities of conditionals and previsions of iterated conditionals. International Journal of Approximate Reasoning 121, 150 - 173. URL: http://www.sciencedirect.com/science/article/pii/S0888613X19304451. doi https://doi.org/10.1016/j.ijar.2020.03.001

[53] Sanfilippo, G., Pfeifer, N., Over, D., Gilio, A., 2018. Probabilistic inferences from conjoined to iterated conditionals. International Journal of Approximate Reasoning 93, 103 - 118. doi 10.1016/j.ijar.2017.10.027

[54] Williams, P., 2007. Notes on conditional previsions. International Journal of Approximate Reasoning 44, 366-383. URL: https://www.sciencedirect.com/science/article/pii/S0888613X06001034. doi/https://doi.org/10.1016/j.ijar.2006.07.019 